\title{
Higgs boson masses and mixings in the complex MSSM with two-loop top-Yukawa-coupling corrections
}

\author{
Wolfgang Hollik and Sebastian Paßehr \\ Max-Planck-Institut für Physik (Werner-Heisenberg-Institut), \\ Föhringer Ring 6, D-80805 München, Germany \\ E-mail: hollik@mpp.mpg.de, passehr@mpp.mpg.de
}

\begin{abstract}
Results for the leading two-loop corrections of $\mathcal{O}\left(\alpha_{t}^{2}\right)$ from the Yukawa sector to the Higgs-boson mass spectrum of the MSSM with complex parameters are presented, with details of the analytical calculation performed in the Feynman-diagrammatic approach using a mixed on-shell/ $\overline{\mathrm{DR}}$ scheme that can be directly matched onto the higher-order terms in the code FeynHiggs. Numerical results are shown for the masses and mixing effects in the neutral Higgs-boson sector and their variation with the phases of the complex parameters. Furthermore, the analytical expressions of the two-loop self-energies and the required renormalization constants are recorded. The new results can consistently be implemented in FeynHiggs.
\end{abstract}

Keywords: Supersymmetry Phenomenology

ARXIV EPRINT: 1409.1687 


\section{Contents}

1 Introduction 1

2 The Higgs sector of the complex MSSM 3

2.1 Tree-level relations for masses and mixing 3

2.2 Masses and mixing beyond lowest order 4

2.3 Renormalized self-energies at the two-loop level 5

3 Subrenormalization $\quad \mathbf{1 2}$

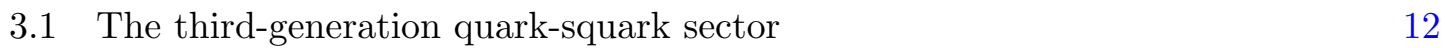

$\begin{array}{lll}3.2 & \text { The chargino-neutralino sector } & 14\end{array}$

4 Numerical results for masses and mixings $\quad 15$

5 Conclusions $\quad 21$

$\begin{array}{ll}\text { A Two-loop mass counterterms } & 21\end{array}$

B Couplings and counterterm insertions $\quad 23$

B.1 Tree-level vertices 23

$\begin{array}{ll}\text { B.2 Counterterm vertices } & 27\end{array}$

C Loop integrals $\quad 30$

C.1 One-loop functions 30

C.2 Two-loop functions 32

D Analytical $\mathcal{O}\left(\alpha_{t}^{2}\right)$ results $\quad 34$

D.1 Symbols and abbreviations 34

D.2 Genuine two-loop self-energies 34

D.3 Genuine two-loop tadpoles 43

D.4 One-loop self-energies with counterterm insertions 43

D.5 One-loop tadpoles with counterterm insertions 46

D.6 Renormalization constants for subrenormalization 47

\section{Introduction}

The recently discovered new boson $[1,2]$ with a mass around $125.6 \mathrm{GeV}$ by the experiments ATLAS and CMS at the LHC has given rise to substantial investigations to reveal the nature of this particle as a Higgs boson responsible for electroweak symmetry breaking. Within the present experimental uncertainties the measured properties of this new boson 
are consistent with the corresponding expectations for the Standard Model Higgs boson [3, 4]; on the other hand, a large variety of other interpretations is possible where the Higgs particle belongs to an extended model connected to physics beyond the Standard Model. Within the theoretically well motivated minimal supersymmetric Standard Model (MSSM), the observed particle could be interpreted as a light state within a richer spectrum of scalar particles. The Higgs sector of the MSSM consists of two complex scalar doublets leading to five physical Higgs bosons and three (would-be) Goldstone bosons. At the tree-level, the physical states are given by the neutral $C P$-even $h, H$ and $C P$-odd $A$ bosons, together with the charged $H^{ \pm}$bosons, and can be parametrized in terms of the $A$-boson mass $m_{A}$ and the ratio of the two vacuum expectation values, $\tan \beta=v_{2} / v_{1}$. In the MSSM with complex parameters, the cMSSM, $C P$-violation is induced in the Higgs sector via loop contributions involving complex parameters from other SUSY sectors leading to mixing between $h, H$ and $A$ in the mass eigenstates [5, 6].

Masses and mixings in the neutral sector are strongly affected by loop contributions. A lot of work has been invested into higher-order calculations of the mass spectrum from the SUSY parameters, in the case of the real MSSM [7-39] as well as for the cMSSM [40-47]. The largest loop contributions originate from the Yukawa sector with the large top Yukawa coupling $h_{t}$, or $\alpha_{t}=h_{t}^{2} /(4 \pi)$. The class of leading two-loop Yukawa-type corrections of $\mathcal{O}\left(\alpha_{t}^{2}\right)$ has been calculated for the case of real parameters [27, 28], applying the method of the effective potential. Together with the full one-loop result [47] and the leading $\mathcal{O}\left(\alpha_{t} \alpha_{s}\right)$ terms [46], both accomplished in the Feynman-diagrammatic approach including complex parameters, it has been implemented in the public program FeynHiggs [11, 29, 47-49]. A calculation of the $\mathcal{O}\left(\alpha_{t}^{2}\right)$ terms for the complex version of the MSSM, however, was not available so far; it is the content of this article.

In a recent paper [50] we have shown first results for the impact of the $\mathcal{O}\left(\alpha_{t}^{2}\right)$ contributions within the cMSSM on the mass of the lightest neutral Higgs boson. Here we give details of the calculation and list the analytic results entering the evaluation of the oneand two-point functions of the Higgs sector and the required counterterms. The calculation is done in the Feynman-diagrammatic approach, extending the on-shell renormalization scheme of ref. [47] to the two-loop level. This ensures that the obtained analytical results for the renormalized two-loop self-energies can consistently be incorporated in FeynHiggs. In the numerical analysis, we show results for the masses and $C P$-mixing of the three neutral Higgs bosons of the cMSSM and their dependence on the complex phases of the relevant parameters.

The paper is organized as follows: section 2 provides the theoretical framework of the calculation and renormalization for getting the dressed propagators of the neutral Higgs sector up to the two-loop level. The necessary one-loop subrenormalization is described in section 3, and numerical results are shown in section 4. The appendix contains all couplings and counterterm vertices needed for the calculation of the contributing Feynman diagrams, as well as a complete list of the counterterms and of the analytical expressions for the tadpoles and the self-energies of the Higgs sector. 


\section{The Higgs sector of the complex MSSM}

\subsection{Tree-level relations for masses and mixing}

The two scalar SU(2)-doublets are conventionally expressed in terms of their components in the following way,

$$
\mathcal{H}_{1}=\left(\begin{array}{c}
v_{1}+\frac{1}{\sqrt{2}}\left(\phi_{1}-i \chi_{1}\right) \\
-\phi_{1}^{-}
\end{array}\right), \quad \mathcal{H}_{2}=\mathrm{e}^{i \xi}\left(\begin{array}{c}
\phi_{2}^{+} \\
v_{2}+\frac{1}{\sqrt{2}}\left(\phi_{2}+i \chi_{2}\right)
\end{array}\right) .
$$

Making use of the notation $\phi_{1}^{-}=\left(\phi_{1}^{+}\right)^{\dagger}, \phi_{2}^{-}=\left(\phi_{2}^{+}\right)^{\dagger}$, the Higgs potential can be written as a polynomial in the field components,

$$
\begin{aligned}
V_{H}= & -T_{\phi_{1}} \phi_{1}-T_{\phi_{2}} \phi_{2}-T_{\chi_{1}} \chi_{1}-T_{\chi_{2}} \chi_{2} \\
& +\frac{1}{2}\left(\phi_{1}, \phi_{2}, \chi_{1}, \chi_{2}\right)\left(\begin{array}{cc}
\mathbf{M}_{\phi} & \mathbf{M}_{\phi \chi} \\
\mathbf{M}_{\phi \chi}^{\dagger} & \mathbf{M}_{\chi}
\end{array}\right)\left(\begin{array}{c}
\phi_{1} \\
\phi_{2} \\
\chi_{1} \\
\chi_{2}
\end{array}\right)+\left(\phi_{1}^{-}, \phi_{2}^{-}\right) \mathbf{M}_{\phi^{ \pm}}\left(\begin{array}{c}
\phi_{1}^{+} \\
\phi_{2}^{+}
\end{array}\right)+\ldots,
\end{aligned}
$$

where the third and fourth powers in the fields have been dropped. Explicit expressions for the tadpole coefficients $T_{i}$ and for the mass matrices $\mathbf{M}$ can be found in ref. [47]. They are parametrized by the phase $\xi$, the real SUSY-breaking quantities $m_{1,2}^{2}=\tilde{m}_{1,2}^{2}+|\mu|^{2}$, and the complex SUSY-breaking quantity $m_{12}^{2}$. The latter can be redefined as real [51] with the help of a Peccei-Quinn transformation [52,53] leaving only the phase $\xi$ as a source of $C P$-violation at the tree-level. The requirement of minimizing $V_{H}$ at the vacuum expectation values $v_{1}$ and $v_{2}$ induces vanishing tadpoles at the tree level, which in turn leads to $\xi=0$. As a consequence, also $\mathbf{M}_{\phi \chi}$ is equal to zero and $\phi_{1,2}$ are decoupled from $\chi_{1,2}$ at the tree-level. The remaining $(2 \times 2)$-matrices $\mathbf{M}_{\phi}, \mathbf{M}_{\chi}, \mathbf{M}_{\phi^{ \pm}}$can be transformed into the mass eigenstate basis with the help of orthogonal matrices $D(x)=\left(\begin{array}{cc}-s_{x} & c_{x} \\ c_{x} & s_{x}\end{array}\right)$, using the abbreviations $s_{x} \equiv \sin x, c_{x} \equiv \cos x$,

$$
\left(\begin{array}{c}
h \\
H
\end{array}\right)=D(\alpha)\left(\begin{array}{l}
\phi_{1} \\
\phi_{2}
\end{array}\right), \quad\left(\begin{array}{l}
A \\
G
\end{array}\right)=D\left(\beta_{n}\right)\left(\begin{array}{l}
\chi_{1} \\
\chi_{2}
\end{array}\right), \quad\left(\begin{array}{c}
H^{ \pm} \\
G^{ \pm}
\end{array}\right)=D\left(\beta_{c}\right)\left(\begin{array}{c}
\phi_{1}^{ \pm} \\
\phi_{2}^{ \pm}
\end{array}\right) .
$$

The Higgs potential in this basis can be expressed as follows,

$$
\begin{aligned}
V_{H}= & -T_{h} h-T_{H} H-T_{A} A-T_{G} G \\
& +\frac{1}{2}(h, H, A, G) \mathbf{M}_{h H A G}\left(\begin{array}{l}
h \\
H \\
A \\
G
\end{array}\right)+\left(H^{-}, G^{-}\right) \mathbf{M}_{H^{ \pm} G^{ \pm}}\left(\begin{array}{l}
H^{+} \\
G^{+}
\end{array}\right)+\ldots
\end{aligned}
$$


with the tadpole coefficients $T_{h, H, A, G}$ and the mass matrices

$$
\mathbf{M}_{h H A G}=\left(\begin{array}{cccc}
m_{h}^{2} & m_{h H}^{2} & m_{h A}^{2} & m_{h G}^{2} \\
m_{h H}^{2} & m_{H}^{2} & m_{H A}^{2} & m_{H G}^{2} \\
m_{h A}^{2} & m_{H A}^{2} & m_{A}^{2} & m_{A G}^{2} \\
m_{h G}^{2} & m_{H G}^{2} & m_{A G}^{2} & m_{G}^{2}
\end{array}\right), \quad \mathbf{M}_{H^{ \pm} G \pm}=\left(\begin{array}{cc}
m_{H^{ \pm}}^{2} & m_{H^{-} G^{+}}^{2} \\
m_{G^{-} H^{+}}^{2} & m_{G^{ \pm}}^{2}
\end{array}\right) ;
$$

explicit expressions for the entries are given in ref. [47].

At lowest order, the tadpoles and the non-diagonal entries of the mass matrices vanish,

$$
\mathbf{M}_{h H A G}^{(0)}=\operatorname{diag}\left(m_{h}^{2}, m_{H}^{2}, m_{A}^{2}, m_{G}^{2}\right), \quad \mathbf{M}_{H^{ \pm} G^{ \pm}}^{(0)}=\operatorname{diag}\left(m_{H^{ \pm}}^{2}, m_{G^{ \pm}}^{2}\right),
$$

for $\beta=\beta_{n}=\beta_{c}$, with $\beta$ given in terms of the vacuum expectations values,

$$
\tan \beta \equiv t_{\beta}=\frac{v_{2}}{v_{1}}
$$

and for the second mixing angle $\alpha$ (with $-\frac{\pi}{2}<\alpha<0$ ) determined by

$$
\tan (2 \alpha)=\frac{m_{A}^{2}+m_{Z}^{2}}{m_{A}^{2}-m_{Z}^{2}} \tan (2 \beta) .
$$

The Goldstone bosons are massless, $m_{G^{ \pm}}=m_{G}=0$, and the masses $m_{H^{ \pm}}, m_{A}, m_{h}, m_{H}$ fullfil the relations

$$
\begin{aligned}
m_{H^{ \pm}}^{2} & =m_{A}^{2}+M_{W}^{2}, \\
m_{h, H}^{2} & =\frac{1}{2}\left(m_{A}^{2}+M_{Z}^{2} \mp \sqrt{\left(m_{A}^{2}+M_{Z}^{2}\right)^{2}-4 m_{A}^{2} M_{Z}^{2} c_{2 \beta}^{2}}\right),
\end{aligned}
$$

including the vector-boson masses $M_{W}$ and $M_{Z}$.

\subsection{Masses and mixing beyond lowest order}

At lowest order, the irreducible two-point vertex functions of the neutral Higgs sector

$$
\Gamma_{h H A G}^{(0)}\left(p^{2}\right)=i\left[p^{2} \mathbf{1}-\mathbf{M}_{h H A G}^{(0)}\right]
$$

are diagonal, and the entries of the mass matrices in eq. (2.6) provide the poles of the diagonal lowest-order propagators

$$
\Delta_{h H A G}^{(0)}\left(p^{2}\right)=-\left[\Gamma_{h H A G}^{(0)}\left(p^{2}\right)\right]^{-1} .
$$

At higher order, the irreducible two-point functions are dressed by adding the renormalized self-energies,

$$
p^{2} \mathbf{1}-\mathbf{M}_{h H A G}^{(0)} \rightarrow p^{2} \mathbf{1}-\mathbf{M}_{h H A G}^{(0)}+\hat{\boldsymbol{\Sigma}}_{h H A G}\left(p^{2}\right) \equiv p^{2} \mathbf{1}-\mathbf{M}_{h H A G}\left(p^{2}\right),
$$

yielding the renormalized two-point vertex functions $\hat{\Gamma}_{h H A G}\left(p^{2}\right)$, which contain in general mixing of all fields with equal quantum numbers. The dressed propagators are obtained accordingly by inverting the matrix $\hat{\Gamma}_{h H A G}\left(p^{2}\right)$. 
In our case, we evaluate the momentum-dependent neutral "mass matrix" in eq. (2.12) at the two-loop level,

$$
\mathbf{M}_{h H A G}^{(2)}\left(p^{2}\right)=\mathbf{M}_{h H A G}^{(0)}-\hat{\boldsymbol{\Sigma}}_{h H A G}^{(1)}\left(p^{2}\right)-\hat{\mathbf{\Sigma}}_{h H A G}^{(2)}(0) .
$$

Therein, $\hat{\mathbf{\Sigma}}_{h H A G}^{(k)}$ denotes the matrix of the renormalized diagonal and non-diagonal selfenergies for the $h, H, A, G$ fields at loop order $k$. For the complex MSSM, the one-loop self-energies are completely known [47], and the leading two-loop $\mathcal{O}\left(\alpha_{t} \alpha_{s}\right)$ contributions have been obtained in the approximation of zero external momentum [46]. Within the same approximation, treating the two-loop self-energies at $p^{2}=0$, we derive the leading Yukawa contributions of $\mathcal{O}\left(\alpha_{t}^{2}\right)$.

In order to obtain the physical Higgs-boson masses from the dressed propagators in the considered approximation, it is sufficient to derive explicitly the entries of the $(3 \times 3)$ submatrix of eq. (2.13) corresponding to the $(h H A)$-components. Mixing with the Goldstone boson yields subleading two-loop contributions; also Goldstone- $Z$ mixing occurs in principle, which is related to the other Goldstone mixings by Slavnov-Taylor identities $[54,55]$ and of subleading type as well [56]. However, mixing with Goldstone bosons has to be taken into account inside the loop diagrams and for a consistent renormalization.

The masses of the three neutral Higgs bosons including the new $\mathcal{O}\left(\alpha_{t}^{2}\right)$ contributions are given by the real parts of the poles of the $h H A$-propagator matrix, obtained as the zeroes of the determinant of the renormalized two-point vertex function,

$$
\operatorname{det} \hat{\Gamma}_{h H A}\left(p^{2}\right)=0, \quad \hat{\Gamma}_{h H A}\left(p^{2}\right)=i\left[p^{2} \mathbf{1}-\mathbf{M}_{h H A}^{(2)}\left(p^{2}\right)\right],
$$

involving the corresponding $(3 \times 3)$-submatrix of eq. $(2.13)$. The impact of the self-energies on the mixing and couplings of the various Higgs bosons can be obtained with he same formalism as described in ref. [47].

\subsection{Renormalized self-energies at the two-loop level}

For obtaining the renormalized self-energies in eq. (2.13), counterterms have to be introduced up to second order in the loop expansion, for the tadpoles

$$
T_{i} \rightarrow T_{i}+\delta^{(1)} T_{i}+\delta^{(2)} T_{i}, \quad i=h, H, A, G,
$$

and for the mass matrices in eq. (2.4),

$$
\begin{aligned}
& \mathbf{M}_{h H A G} \rightarrow \mathbf{M}_{h H A G}^{(0)}+\delta^{(1)} \mathbf{M}_{h H A G}+\delta^{(2)} \mathbf{M}_{h H A G}, \\
& \delta^{(k)} \mathbf{M}_{h H A G}=\left(\begin{array}{cccc}
\delta^{(k)} m_{h}^{2} & \delta^{(k)} m_{h H}^{2} & \delta^{(k)} m_{h A}^{2} & \delta^{(k)} m_{h G}^{2} \\
\delta^{(k)} m_{H h}^{2} & \delta^{(k)} m_{H}^{2} & \delta^{(k)} m_{H A}^{2} & \delta^{(k)} m_{H G}^{2} \\
\delta^{(k)} m_{A h}^{2} & \delta^{(k)} m_{A H}^{2} & \delta^{(k)} m_{A}^{2} & \delta^{(k)} m_{A G}^{2} \\
\delta^{(k)} m_{G h}^{2} & \delta^{(k)} m_{G H}^{2} & \delta^{(k)} m_{G A}^{2} & \delta^{(k)} m_{G}^{2}
\end{array}\right), \\
& \mathbf{M}_{H^{ \pm} G^{ \pm}} \rightarrow \mathbf{M}_{H^{ \pm} G^{ \pm}}^{(0)}+\delta^{(1)} \mathbf{M}_{H^{ \pm} G^{ \pm}}+\delta^{(2)} \mathbf{M}_{H^{ \pm} G^{ \pm}}, \\
& \delta^{(k)} \mathbf{M}_{H^{ \pm} G^{ \pm}}=\left(\begin{array}{cc}
\delta^{(k)} m_{H^{ \pm}}^{2} & \delta^{(k)} m_{H^{ \pm} G^{ \pm}}^{2} \\
\delta^{(k)} m_{G^{ \pm} H^{ \pm}}^{2} & \delta^{(k)} m_{G^{ \pm}}^{2}
\end{array}\right) .
\end{aligned}
$$


For getting the proper counterterms for the mass matrices in eq. (2.5) one has to distinguish between the rotation angles $\beta_{n}, \beta_{c}$ from eqs. (2.3) and $\beta$ in eq. (2.7) when generating the expressions for the matrix elements in eq. (2.5). Whereas $\alpha, \beta_{n}$ and $\beta_{c}$ are not renormalized, $\beta$ gets counterterms $\beta \rightarrow \beta+\delta \beta$ according to the renormalization of $\tan \beta$,

$$
t_{\beta} \rightarrow t_{\beta}+\delta^{(1)} t_{\beta}+\delta^{(2)} t_{\beta}
$$

In the resulting expressions for the counterterm matrices, the identification $\beta_{c}=\beta_{n}=\beta$ is done afterwards at each order. Details at the one-loop level can be found in ref. [47].

In addition to the parameter renormalization described above, field-renormalization constants $Z_{\mathcal{H}_{i}}=1+\delta^{(1)} Z_{\mathcal{H}_{i}}+\delta^{(2)} Z_{\mathcal{H}_{i}}$ are introduced up to two-loop order for each of the scalar doublets of eqs. (2.1) through the transformation

$$
\begin{aligned}
\mathcal{H}_{i} \rightarrow \sqrt{Z_{\mathcal{H}_{i}}} \mathcal{H}_{i} & =\left[1+\frac{1}{2} \delta^{(1)} Z_{\mathcal{H}_{i}}+\frac{1}{2} \Delta^{(2)} Z_{\mathcal{H}_{i}}\right] \mathcal{H}_{i}, \\
\Delta^{(2)} Z_{\mathcal{H}_{i}} & =\delta^{(2)} Z_{\mathcal{H}_{i}}-\frac{1}{4}\left(\delta^{(1)} Z_{\mathcal{H}_{i}}\right)^{2} .
\end{aligned}
$$

The field-renormalization constants in the mass-eigenstate basis of eqs. (2.3) are obtained by

$$
\begin{gathered}
\left(\begin{array}{l}
h \\
H
\end{array}\right) \rightarrow D(\alpha)\left(\begin{array}{cc}
\sqrt{Z_{\mathcal{H}_{1}}} & 0 \\
0 & \sqrt{Z_{\mathcal{H}_{2}}}
\end{array}\right) D(\alpha)^{-1}\left(\begin{array}{l}
h \\
H
\end{array}\right) \equiv \mathbf{Z}_{h H}\left(\begin{array}{l}
h \\
H
\end{array}\right), \\
\left(\begin{array}{l}
A \\
G
\end{array}\right) \rightarrow D\left(\beta_{n}\right)\left(\begin{array}{cc}
\sqrt{Z_{\mathcal{H}_{1}}} & 0 \\
0 & \sqrt{Z_{\mathcal{H}_{2}}}
\end{array}\right) D\left(\beta_{n}\right)^{-1}\left(\begin{array}{l}
A \\
G
\end{array}\right) \equiv \mathbf{Z}_{A G}\left(\begin{array}{l}
A \\
G
\end{array}\right), \\
\left(\begin{array}{l}
H^{ \pm} \\
G^{ \pm}
\end{array}\right) \rightarrow D\left(\beta_{c}\right)\left(\begin{array}{cc}
\sqrt{Z_{\mathcal{H}_{1}}} & 0 \\
0 & \sqrt{Z_{\mathcal{H}_{2}}}
\end{array}\right) D\left(\beta_{c}\right)^{-1}\left(\begin{array}{l}
H^{ \pm} \\
G^{ \pm}
\end{array}\right) \equiv \mathbf{Z}_{H^{ \pm} G^{ \pm}}\left(\begin{array}{l}
H^{ \pm} \\
G^{ \pm}
\end{array}\right) .
\end{gathered}
$$

One-loop expressions for the entries in the $\mathbf{Z}$-matrices in eqs. (2.20) are given in in ref. [47]; their extension up to two-loop order is listed in the following,

$$
\begin{aligned}
& \mathbf{Z}_{h H}=\mathbf{1}+\delta^{(1)} \mathbf{Z}_{h H}+\delta^{(2)} \mathbf{Z}_{h H}, \quad \delta^{(i)} \mathbf{Z}_{h H}=\frac{1}{2}\left(\begin{array}{cc}
\delta^{(i)} Z_{h h} & \delta^{(i)} Z_{h H} \\
\delta^{(i)} Z_{H h} & \delta^{(i)} Z_{H H}
\end{array}\right), \\
& \delta^{(2)} Z_{h h}=\left(s_{\alpha}^{2} \Delta^{(2)} Z_{\mathcal{H}_{1}}+c_{\alpha}^{2} \Delta^{(2)} Z_{\mathcal{H}_{2}}\right), \\
& \delta^{(2)} Z_{H H}=\left(c_{\alpha}^{2} \Delta^{(2)} Z_{\mathcal{H}_{1}}+s_{\alpha}^{2} \Delta^{(2)} Z_{\mathcal{H}_{2}}\right), \\
& \delta^{(2)} Z_{h H}=\delta^{(2)} Z_{H h}=c_{\alpha} s_{\alpha}\left(\Delta^{(2)} Z_{\mathcal{H}_{2}}-\Delta^{(2)} Z_{\mathcal{H}_{1}}\right), \\
& \mathbf{Z}_{A G}=\mathbf{1}+\delta^{(1)} \mathbf{Z}_{A G}+\delta^{(2)} \mathbf{Z}_{A G}, \quad \delta^{(i)} \mathbf{Z}_{A G}=\frac{1}{2}\left(\begin{array}{c}
\delta^{(i)} Z_{A A} \delta^{(i)} Z_{A G} \\
\delta^{(i)} Z_{G A} \delta^{(i)} Z_{G G}
\end{array}\right), \\
& \delta^{(2)} Z_{A A}=\left(s_{\beta_{n}}^{2} \Delta^{(2)} Z_{\mathcal{H}_{1}}+c_{\beta_{n}}^{2} \Delta^{(2)} Z_{\mathcal{H}_{2}}\right),
\end{aligned}
$$




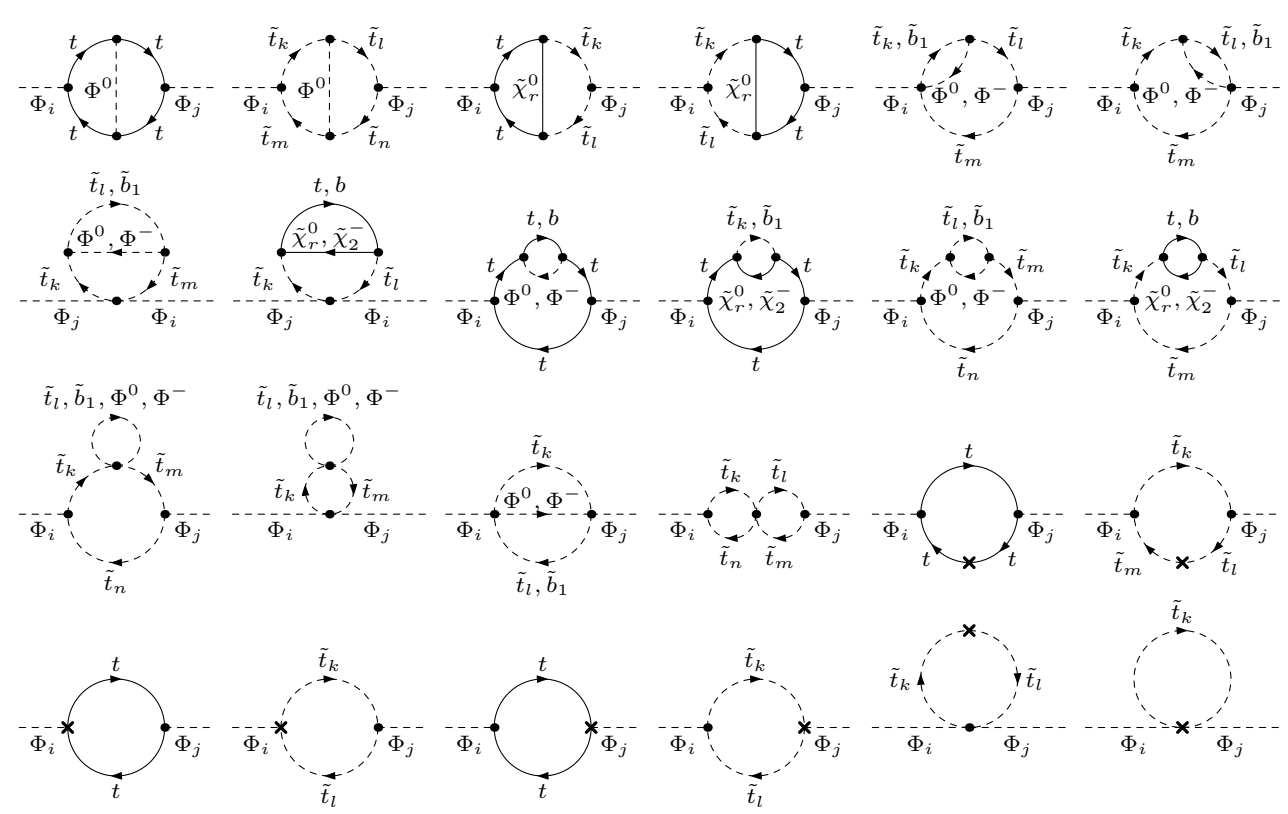

Figure 1. List of two-loop self-energy diagrams for the neutral Higgs bosons. One-loop counterterm insertions are denoted by a cross. $\Phi_{i}=h, H, A ; \Phi^{0}=h, H, A, G ; \Phi^{-}=H^{-}, G^{-}$.

$$
\begin{aligned}
& \delta^{(2)} Z_{G G}=\left(c_{\beta_{n}}^{2} \Delta^{(2)} Z_{\mathcal{H}_{1}}+s_{\beta_{n}}^{2} \Delta^{(2)} Z_{\mathcal{H}_{2}}\right), \\
& \delta^{(2)} Z_{A G}=\delta^{(2)} Z_{G A}=c_{\beta_{n}} s_{\beta_{n}}\left(\Delta^{(2)} Z_{\mathcal{H}_{2}}-\Delta^{(2)} Z_{\mathcal{H}_{1}}\right), \\
& \mathbf{Z}_{H^{ \pm} G^{ \pm}}=\mathbf{1}+\delta^{(1)} \mathbf{Z}_{H^{ \pm} G^{ \pm}}+\delta^{(2)} \mathbf{Z}_{H^{ \pm} G^{ \pm}}, \quad \delta^{(i)} \mathbf{Z}_{H^{ \pm} G^{ \pm}}=\frac{1}{2}\left(\begin{array}{c}
\delta^{(i)} Z_{H^{ \pm} H^{ \pm}} \delta^{(i)} Z_{H^{ \pm} G^{ \pm}} \\
\delta^{(i)} Z_{G^{ \pm} H^{ \pm}} \delta^{(i)} Z_{G^{ \pm} G^{ \pm}}
\end{array}\right),
\end{aligned}
$$

$$
\begin{aligned}
\delta^{(2)} Z_{H^{ \pm} H^{ \pm}} & =\left(s_{\beta_{c}}^{2} \Delta^{(2)} Z_{\mathcal{H}_{1}}+c_{\beta_{c}}^{2} \Delta^{(2)} Z_{\mathcal{H}_{2}}\right), \\
\delta^{(2)} Z_{G^{ \pm} G^{ \pm}} & =\left(c_{\beta_{c}}^{2} \Delta^{(2)} Z_{\mathcal{H}_{1}}+s_{\beta_{c}}^{2} \Delta^{(2)} Z_{\mathcal{H}_{2}}\right), \\
\delta^{(2)} Z_{H^{ \pm} G^{ \pm}} & =\delta^{(2)} Z_{G^{ \pm} H^{ \pm}}=c_{\beta_{c}} s_{\beta_{c}}\left(\Delta^{(2)} Z_{\mathcal{H}_{2}}-\Delta^{(2)} Z_{\mathcal{H}_{1}}\right) .
\end{aligned}
$$

The next step is the determination of the renormalized self-energies $\hat{\boldsymbol{\Sigma}}$. The one-loop self-energies $\hat{\boldsymbol{\Sigma}}_{h H A G}^{(1)}\left(p^{2}\right)$ are contained in ref. [47]. The renormalized two-loop self-energies can be written in compact matrix notation as follows,

$$
\hat{\boldsymbol{\Sigma}}_{h H A G}^{(2)}\left(p^{2}\right)=\boldsymbol{\Sigma}_{h H A G}^{(2)}\left(p^{2}\right)-\delta^{(2)} \mathbf{M}_{h H A G}^{\mathbf{Z}},
$$

where $\boldsymbol{\Sigma}_{h H A G}^{(2)}$ denotes the unrenormalized self-energies corresponding to the sum of the genuine two-loop diagrams and one-loop diagrams with subrenormalization. The symbol $\delta^{(2)} \mathbf{M}_{h H A G}^{\mathbf{Z}}$ comprises all the two-loop counterterms for $\boldsymbol{\Sigma}_{h H A G}^{(2)}$, resulting from parameter 
and field renormalization,

$$
\begin{aligned}
\delta^{(2)} \mathbf{M}_{h H A G}^{\mathbf{Z}}= & \left(\begin{array}{cc}
\delta^{(2)} \mathbf{Z}_{h H}^{T} & \mathbf{0} \\
\mathbf{0} & \delta^{(2)} \mathbf{Z}_{A G}^{T}
\end{array}\right)\left(\mathbf{M}_{h H A G}^{(0)}-p^{2} \mathbf{1}\right)+\left(\mathbf{M}_{h H A G}^{(0)}-p^{2} \mathbf{1}\right)\left(\begin{array}{cc}
\delta^{(2)} \mathbf{Z}_{h H} & \mathbf{0} \\
\mathbf{0} & \delta^{(2)} \mathbf{Z}_{A G}
\end{array}\right) \\
& +\left(\begin{array}{cc}
\delta^{(1)} \mathbf{Z}_{h H}^{T} & \mathbf{0} \\
\mathbf{0} & \delta^{(1)} \mathbf{Z}_{A G}^{T}
\end{array}\right) \delta^{(1)} \mathbf{M}_{h H A G}+\delta^{(1)} \mathbf{M}_{h H A G}\left(\begin{array}{cc}
\delta^{(1)} \mathbf{Z}_{h H} & \mathbf{0} \\
\mathbf{0} & \delta^{(1)} \mathbf{Z}_{A G}
\end{array}\right) \\
& +\left(\begin{array}{cc}
\delta^{(1)} \mathbf{Z}_{h H}^{T} & \mathbf{0} \\
\mathbf{0} & \delta^{(1)} \mathbf{Z}_{A G}^{T}
\end{array}\right) \mathbf{M}_{h H A G}^{(0)}\left(\begin{array}{cc}
\delta^{(1)} \mathbf{Z}_{h H} & \mathbf{0} \\
\mathbf{0} & \delta^{(1)} \mathbf{Z}_{A G}
\end{array}\right)+\delta^{(2)} \mathbf{M}_{h H A G} \cdot(2.23)
\end{aligned}
$$

Besides the field-renormalization constants from eqs. (2.21), and products of one-loop quantities, the two-loop mass counterterms are required, which are derived from the Higgs potential. A complete list is given in appendix A; this list is valid for the general two-loop case and not restricted to the Yukawa approximation.

For the concrete calculation of the quantities entering eq. (2.14) we evaluate the twoloop self-energies at $p^{2}=0$ in the top-Yukawa approximation, which neglects contributions from the gauge sector (gaugeless limit) as well as the Yukawa coupling of the bottom quark setting the $b$-quark mass to zero. In this approximation, only those Feynman diagrams that are depicted in figure 1 are calculated. The couplings utilized for their evaluation are listed in appendix B. The diagrammatic calculation of the self-energies has been performed with FeynArts [57] for the generation of the Feynman diagrams and TwoCalc [58] for the two-loop tensor reduction and trace evaluation. The renormalization constants have been obtained with the help of FormCalc [59]. The analytical result for the contribution from the genuine two-loop diagrams can be found in appendix D.2, and the result from the diagrams with subrenormalization in appendix D.4.

In our approximation, the required two-loop mass counterterms are simplifications of those in appendix A and read as follows,

$$
\begin{aligned}
\delta^{(2)} m_{h}^{2}= & m_{H^{ \pm}}^{2} c_{\beta}^{4}\left(\delta^{(1)} t_{\beta}\right)^{2}-\frac{e}{2 M_{W} s_{\mathrm{w}}} c_{\beta}^{2} \delta^{(1)} t_{\beta} \delta^{(1)} T_{H} \\
& -\frac{e}{2 M_{W} s_{\mathrm{w}}}\left[\delta^{(2)} T_{h}+\delta^{(1)} T_{h} \delta^{(1)} Z_{\mathrm{w}}\right], \\
\delta^{(2)} m_{H}^{2}= & \delta^{(2)} m_{H^{ \pm}}^{2}, \\
\delta^{(2)} m_{A}^{2}= & \delta^{(2)} m_{H^{ \pm}}^{2}, \\
\delta^{(2)} m_{h H}^{2}= & m_{H^{ \pm}}^{2} c_{\beta}^{2} \delta^{(2)} t_{\beta}+c_{\beta}^{2} \delta^{(1)} m_{H^{ \pm}}^{2} \delta^{(1)} t_{\beta}-m_{H^{ \pm}}^{2} c_{\beta}^{3} s_{\beta}\left(\delta^{(1)} t_{\beta}\right)^{2} \\
& -\frac{e}{2 M_{W} s_{\mathrm{w}}}\left[\delta^{(2)} T_{H}+\delta^{(1)} T_{H} \delta^{(1)} Z_{\mathrm{w}}\right], \\
\delta^{(2)} m_{h A}^{2}= & -\frac{e}{2 M_{W} s_{\mathrm{w}}}\left[\delta^{(2)} T_{A}+\delta^{(1)} T_{A} \delta^{(1)} Z_{\mathrm{w}}\right], \\
\delta^{(2)} m_{H A}^{2}= & 0 .
\end{aligned}
$$

The quantities $\delta^{(1)} e, \delta^{(1)} M_{W}$ and $\delta^{(1)} s_{\mathrm{w}}$ always occur in terms of the combination

$$
\delta^{(1)} Z_{\mathrm{w}}=\frac{\delta^{(1)} e}{e}-\frac{\delta^{(1)} M_{W}}{M_{W}}-\frac{\delta^{(1)} s_{\mathrm{w}}}{s_{\mathrm{w}}} ;
$$


where, however, in the gauge-less limit $\delta^{(1)} e$ is equal to zero. The other elements of $\delta^{(2)} \mathbf{M}_{h H A G}$ not listed are determined by symmetry (see appendix A), or they involve mixing with the Goldstone boson $G$, which is not needed for eq. (2.14). Furthermore, in the gaugeless limit the mass relations in eq. (2.9) simplify to $m_{h}^{2}=0, m_{H}^{2}=m_{A}^{2}, m_{H^{ \pm}}^{2}=m_{A}^{2}$, together with $m_{G}^{2}=0, m_{G^{ \pm}}^{2}=0$, and the mixing angle $\alpha$ is restricted by the relations $s_{\alpha}=-c_{\beta}$ and $c_{\alpha}=s_{\beta}$.

In eqs. (2.23) also several one-loop mass counterterms are needed, which in the present approximations are given by the following expressions (symmetric in the neutral indices $h, H, A, G)$,

$$
\begin{aligned}
\delta^{(1)} m_{h}^{2} & =-\frac{e}{2 s_{\mathrm{w}} M_{W}} \delta^{(1)} T_{h}, \\
\delta^{(1)} m_{H}^{2} & =\delta^{(1)} m_{H^{ \pm}}^{2}, \\
\delta^{(1)} m_{A}^{2} & =\delta^{(1)} m_{H^{ \pm}}^{2}, \\
\delta^{(1)} m_{h H}^{2} & =-\frac{e}{2 s_{\mathrm{w}} M_{W}} \delta^{(1)} T_{H}+m_{H^{ \pm}}^{2} c_{\beta}^{2} \delta^{(1)} t_{\beta}, \\
\delta^{(1)} m_{h A}^{2} & =-\frac{e}{2 s_{\mathrm{w}} M_{W}} \delta^{(1)} T_{A}, \\
\delta^{(1)} m_{h G}^{2} & =0, \\
\delta^{(1)} m_{H A}^{2} & =0, \\
\delta^{(1)} m_{H G}^{2} & =\delta^{(1)} m_{h A}^{2}, \\
\delta^{(1)} m_{A G}^{2} & =-\frac{e}{2 s_{\mathrm{w}} M_{W}} \delta^{(1)} T_{H}-m_{A}^{2} c_{\beta} s_{\beta} \delta^{(1)} t_{\beta}, \\
\delta^{(1)} m_{H^{-} G^{+}}^{2} & =\frac{e}{2 s_{\mathrm{w}} M_{W}}\left[\delta^{(1)} T_{H}-i \delta^{(1)} T_{A}\right]-m_{H^{ \pm}}^{2} c_{\beta}^{2} \delta^{(1)} t_{\beta}, \\
\delta^{(1)} m_{G^{-} H^{+}}^{2} & =\left(\delta^{(1)} m_{H^{-} G^{+}}^{2}\right)^{*} .
\end{aligned}
$$

The renormalization constants in eqs. (2.15)-(2.17) are determined via renormalization conditions that are extended from the one-loop level, as specified in ref. [47], to two-loop order; explicit expressions for the renormalization constants are given in appendix D.6:

- The tadpole counterterms $\delta^{(k)} T_{i}$ are fixed by requiring that the minimum of the Higgs potential is not shifted, which means that the tadpole coefficients have to vanish at each order, ${ }^{1}$

$$
T_{i}^{(1)}+\delta^{(1)} T_{i}=0, \quad T_{i}^{(2)}+\delta^{(2)} T_{i}^{Z}=0, \quad i=h, H, A,
$$

where

$$
\begin{aligned}
& \left(\delta^{(2)} T_{h}^{Z}, \delta^{(2)} T_{H}^{Z}\right)=\left(\delta^{(1)} T_{h}, \delta^{(1)} T_{H}\right) \delta^{(1)} \mathbf{Z}_{h H}+\left(\delta^{(2)} T_{h}, \delta^{(2)} T_{H}\right) \\
& \left(\delta^{(2)} T_{A}^{Z}, \delta^{(2)} T_{G}^{Z}\right)=\left(\delta^{(1)} T_{A}, \delta^{(1)} T_{G}\right) \delta^{(1)} \mathbf{Z}_{A G}+\left(\delta^{(2)} T_{A}, \delta^{(2)} T_{G}\right)
\end{aligned}
$$

\footnotetext{
${ }^{1}$ The counterterms $\delta^{(k)} T_{G}$ are not independent and do not need separate renormalization conditions.
} 


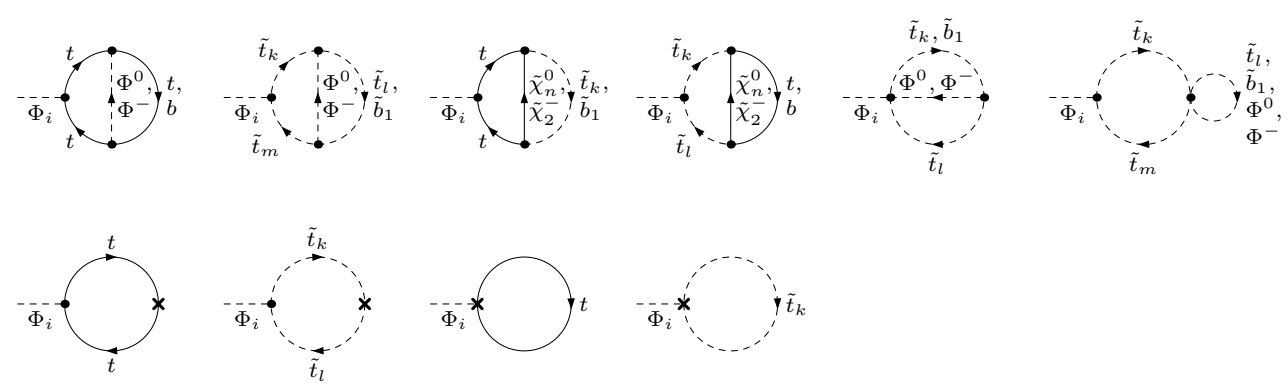

Figure 2. List of two-loop tadpole diagrams contributing to $T_{i}^{(2)}$. One-loop counterterm insertions are denoted by a cross. $\Phi_{i}=h, H, A ; \Phi^{0}=h, H, A, G ; \Phi^{-}=H^{-}, G^{-}$.

$T_{i}^{(k)}$ denote the unrenormalized one-point vertex functions at one- and two-loop order; the two-loop diagrams contributing to $T_{i}^{(2)}$ are displayed in figure 2 and written down in appendix D.3 and appendix D.5. The relation for the mixing angles $\beta_{n}=\beta_{c}=\beta$ is a consequence of the tapole conditions $T_{i}=0$ at lowest order.

- The charged Higgs-boson mass $m_{H^{ \pm}}$is the only independent mass parameter of the Higgs sector and is used as an input quantity. Accordingly, the corresponding mass counterterm is fixed by an independent renormalization condition, chosen as on-shell condition, which in the $p^{2}=0$ approximation is given by $\Re \mathfrak{e}\left[\hat{\Sigma}_{H^{ \pm}}^{(k)}(0)\right]=0$ for the renormalized charged-Higgs self-energy, at the two-loop level specified in terms of the unrenormalized charged self-energies (the contributing Feynman diagrams are shown in figure 3) and respective counterterms,

$$
\hat{\Sigma}_{H^{ \pm}}^{(2)}=\left(\hat{\boldsymbol{\Sigma}}_{H^{ \pm} G^{ \pm}}^{(2)}\right)_{11}, \quad \hat{\boldsymbol{\Sigma}}_{H^{ \pm} G^{ \pm}}^{(2)}(0)=\boldsymbol{\Sigma}_{H^{ \pm} G^{ \pm}}^{(2)}(0)-\delta^{(2)} \mathbf{M}_{H^{ \pm} G^{ \pm}}^{\mathbf{Z}},
$$

with

$$
\begin{aligned}
\delta^{(2)} \mathbf{M}_{H^{ \pm} G^{ \pm}}^{\mathbf{Z}}= & \delta^{(2)} \mathbf{Z}_{H^{ \pm} G^{ \pm}}^{T} \mathbf{M}_{H^{ \pm} G^{ \pm}}^{(0)}+\mathbf{M}_{H^{ \pm} G^{ \pm}}^{(0)} \delta^{(2)} \mathbf{Z}_{H^{ \pm} G^{ \pm}} \\
& +\delta^{(1)} \mathbf{Z}_{H^{ \pm} G^{ \pm}}^{T} \delta^{(1)} \mathbf{M}_{H^{ \pm} G^{ \pm}}+\delta^{(1)} \mathbf{M}_{H^{ \pm} G^{ \pm}} \delta^{(1)} \mathbf{Z}_{H^{ \pm} G^{ \pm}} \\
& +\delta^{(1)} \mathbf{Z}_{H^{ \pm} G^{ \pm}}^{T} \mathbf{M}_{H^{ \pm} G^{ \pm}}^{(0)} \delta^{(1)} \mathbf{Z}_{H^{ \pm} G^{ \pm}}+\delta^{(2)} \mathbf{M}_{H^{ \pm} G^{ \pm}}
\end{aligned}
$$

The independent mass counterterm $\delta^{(2)} m_{H^{ \pm}}^{2}=\left(\delta^{(2)} \mathbf{M}_{H^{ \pm} G^{ \pm}}\right)_{11}$ can be extracted from the on-shell condition, yielding

$$
\begin{aligned}
\delta^{(2)} m_{H^{ \pm}}^{2}= & \Re \mathfrak{e}\left[\Sigma_{H^{ \pm}}^{(2)}(0)\right]-\Re \mathfrak{e}\left[\delta^{(1)} m_{H^{ \pm} H^{ \pm}} \delta^{(1)} Z_{H^{ \pm} H^{ \pm}}+\delta^{(1)} m_{H^{ \pm} G^{ \pm}} \delta^{(1)} Z_{H^{ \pm} G^{ \pm}}\right] \\
& -\Re \mathfrak{e}\left[m_{H^{ \pm}}^{2}\left(\left(\frac{1}{2} \delta^{(1)} Z_{H^{ \pm} H^{ \pm}}\right)^{2}+\delta^{(2)} Z_{H^{ \pm} H^{ \pm}}\right)\right] .
\end{aligned}
$$

The result for the charged Higgs-boson self-energy can be found in appendix D.2 and appendix D.4.

- The field-renormalization constants of the Higgs mass eigenstates in eq. (2.20) are combinations of the basic doublet-field renormalization constants $\delta^{(k)} Z_{\mathcal{H}_{1}}$ and $\delta^{(k)} Z_{\mathcal{H}_{2}}$ 

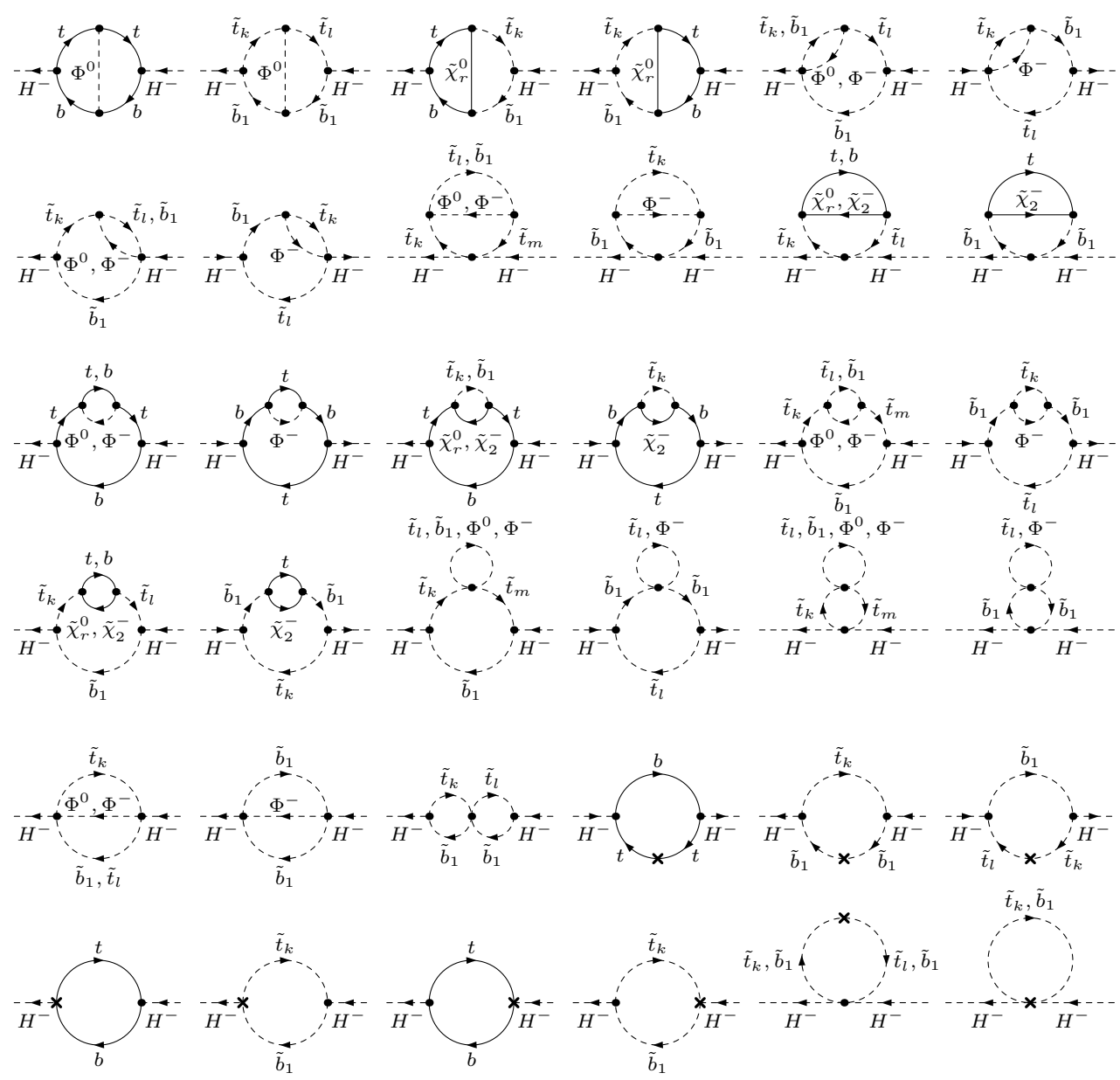

Figure 3. List of two-loop self-energy diagrams for the charged Higgs bosons. One-loop counterterm insertions are denoted by a cross. $\Phi^{0}=h, H, A, G ; \Phi^{-}=H^{-}, G^{-}$.

$(k=1,2)$, which are fixed by the $\overline{\mathrm{DR}}$-conditions for the derivatives of the corresponding self-energies,

$$
\delta^{(k)} Z_{\mathcal{H}_{1}}=-\left[\Sigma_{H H}^{(k)^{\prime}}(0)\right]_{\alpha=0}^{\operatorname{div}}, \quad \delta^{(k)} Z_{\mathcal{H}_{2}}=-\left[\Sigma_{h h}^{(k)^{\prime}}(0)\right]_{\alpha=0}^{\operatorname{div}} .
$$

- $t_{\beta} \equiv \tan \beta$ is renormalized in the $\overline{\mathrm{DR}}$-scheme, which has been shown to be a very convenient choice [60] (alternative process-dependent definitions and renormalization of $t_{\beta}$ can be found in ref. [54]). It has been clarified in refs. [61, 62] that the counterterm for $k=1,2$ can be written as

$$
\delta^{(k)} t_{\beta}^{2}=t_{\beta}^{2}\left(\delta^{(k)} Z_{\mathcal{H}_{2}}-\delta^{(k)} Z_{\mathcal{H}_{1}}\right),
$$

which at the two-loop level, however, is a special feature of our approximation and not generally valid.

- In the on-shell scheme, also the counterterms $\delta^{(1)} M_{W}^{2} / M_{W}^{2}$ and $\delta^{(1)} M_{Z}^{2} / M_{Z}^{2}$ appear, which are required for renormalization of the top Yukawa coupling $h_{t}=\left(e m_{t}\right) /\left(\sqrt{2} s_{\beta} s_{\mathrm{w}} M_{W}\right)$. 
Also in the gauge-less limit these ratios have finite and divergent contributions arising from the Yukawa couplings and thus have to be included as one-loop quantities $\sim h_{t}^{2}$; they are evaluated from the $W$ and $Z$ self-energies yielding

$$
\frac{\delta^{(1)} M_{W}^{2}}{M_{W}^{2}}=\frac{\Sigma_{W}^{(1)}(0)}{M_{W}^{2}}, \quad \frac{\delta^{(1)} M_{Z}^{2}}{M_{Z}^{2}}=\frac{\Sigma_{Z}^{(1)}(0)}{M_{Z}^{2}}, \quad \delta^{(1)} s_{\mathrm{w}}^{2}=c_{\mathrm{w}}^{2}\left(\frac{\delta^{(1)} M_{Z}^{2}}{M_{Z}^{2}}-\frac{\delta^{(1)} M_{W}^{2}}{M_{W}^{2}}\right) .
$$

In the Yukawa approximation, $\delta^{(1)} s_{\mathrm{w}}^{2}$ is finite. The corresponding Feynman graphs are contained in figure 5 .

The appearance of the finite quantity $\delta^{(1)} s_{\mathrm{w}}^{2}$ in the $\mathcal{O}\left(\alpha_{t}^{2}\right)$ terms is a consequence of the on-shell scheme where the top-Yukawa coupling $h_{t}=m_{t} / v_{2}=m_{t} /\left(v s_{\beta}\right)$ is expressed in terms of the parameters

$$
\frac{1}{v}=\frac{g_{\mathrm{w}}}{\sqrt{2} M_{W}}=\frac{e}{\sqrt{2} s_{\mathrm{w}} M_{W}} .
$$

Accordingly, also the one-loop self-energies have to be parametrized in terms of this representation for $h_{t}$ when added to the two-loop self-energies in eq. (2.13). On the other hand, it may be convenient to use the Fermi constant $G_{\mathrm{F}}$ for parametrization of the one-loop self-energies; in that case the relation

$$
\sqrt{2} G_{\mathrm{F}}=\frac{e^{2}}{4 s_{\mathrm{w}}^{2} M_{W}^{2}}\left(1+\Delta^{(1)} r\right),
$$

has to be applied, which is affected by loop contributions also in the gaugeless limit, described by the one-loop quantity

$$
\Delta^{(1)} r=-\frac{c_{\mathrm{w}}^{2}}{s_{\mathrm{w}}^{2}}\left(\frac{\delta^{(1)} M_{Z}^{2}}{M_{Z}^{2}}-\frac{\delta^{(1)} M_{W}^{2}}{M_{W}^{2}}\right)=-\frac{\delta^{(1)} s_{\mathrm{w}}^{2}}{s_{\mathrm{w}}^{2}} .
$$

This finite shift in the one-loop self-energies induces two-loop terms of $\mathcal{O}\left(\alpha_{t}^{2}\right)$, effectively cancelling all other occurrences of $\delta^{(1)} s_{\mathrm{w}}^{2}$.

\section{Subrenormalization}

The two-loop top Yukawa coupling contributions to the self-energies and tadpoles involve one-loop diagrams with insertions of one-loop counterterms. This subrenormalization concerns masses and couplings in the colored sector and in the chargino-neutralino sector.

\subsection{The third-generation quark-squark sector}

The required one-loop counterterms for subrenormalization arise from the top and scalar top $(\tilde{t})$ as well as scalar bottom $(\tilde{b})$ sectors. The stop and sbottom mass matrices in the $\left(\tilde{t}_{\mathrm{L}}, \tilde{t}_{\mathrm{R}}\right)$ and $\left(\tilde{b}_{\mathrm{L}}, \tilde{b}_{\mathrm{R}}\right)$ bases are given by

$$
\mathbf{M}_{\tilde{q}}=\left(\begin{array}{cc}
m_{\tilde{q}_{\mathrm{L}}}^{2}+m_{q}^{2}+M_{Z}^{2} c_{2 \beta}\left(T_{q}^{3}-Q_{q} s_{\mathrm{w}}^{2}\right) & m_{q}\left(A_{q}^{*}-\mu \kappa_{q}\right) \\
m_{q}\left(A_{q}-\mu^{*} \kappa_{q}\right) & m_{\tilde{q}_{\mathrm{R}}}^{2}+m_{q}^{2}+M_{Z}^{2} c_{2 \beta} Q_{q} s_{\mathrm{w}}^{2}
\end{array}\right), \quad \kappa_{t}=\frac{1}{t_{\beta}}, \quad \kappa_{b}=t_{\beta},
$$




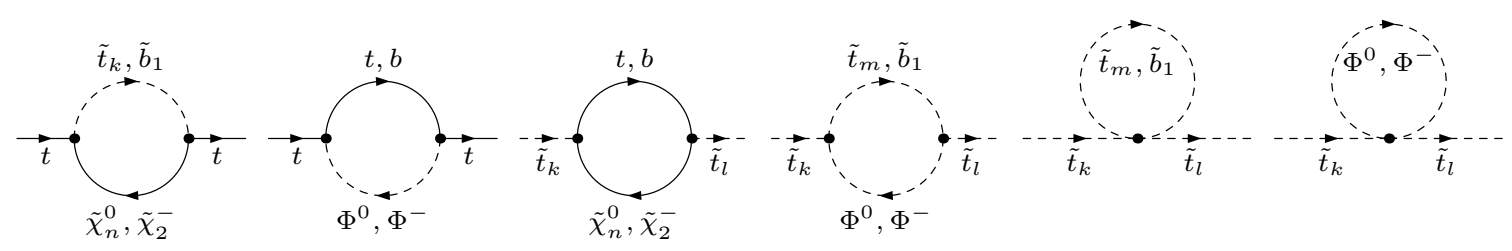

Figure 4. Feynman diagrams for renormalization of the quark-squark sector. $\Phi^{0}=h, H, A, G$; $\Phi^{-}=H^{-}, G^{-}$.

with $Q_{q}$ and $T_{q}^{3}$ denoting charge and isospin of $q=t, b$. $\mathrm{SU}(2)$-invariance requires $m_{\tilde{t}_{\mathrm{L}}}^{2}=m_{\tilde{b}_{\mathrm{L}}}^{2} \equiv m_{\tilde{q}_{3}}^{2}$. In the gaugeless approximation the $D$-terms vanish in both the $\tilde{t}$ and $\tilde{b}$ matrices. Moreover, in our approximation the $b$-quark is treated as massless; hence, the off-diagonal entries of the sbottom matrix are zero and the mass eigenvalues can be read off directly, $m_{\tilde{b}_{1}}^{2}=m_{\tilde{b}_{\mathrm{L}}}^{2}=m_{\tilde{q}_{3}}^{2}, m_{\tilde{b}_{2}}^{2}=m_{\tilde{b}_{\mathrm{R}}}^{2}$. The stop mass eigenvalues can be obtained by performing a unitary transformation,

$$
\mathbf{U}_{\tilde{t}} \mathbf{M}_{\tilde{t}} \mathbf{U}_{\tilde{t}}^{\dagger}=\operatorname{diag}\left(m_{\tilde{t}_{1}}^{2}, m_{\tilde{t}_{2}}^{2}\right) .
$$

Since $A_{t}$ and $\mu$ are complex parameters in general, the unitary matrix $\mathbf{U}_{\tilde{t}}$ consists of one mixing angle $\theta_{\tilde{t}}$ and one phase $\varphi_{\tilde{t}}$.

Five independent parameters are introduced by the quark-squark sector, which enter the two-loop calculation in addition to those of the previous section: the top mass $m_{t}$, the soft SUSY-breaking parameters $m_{\tilde{q}_{3}}$ and $m_{\tilde{t}_{\mathrm{R}}}\left(m_{\tilde{b}_{\mathrm{R}}}\right.$ decouples for $\left.m_{b}=0\right)$, and the complex mixing parameter $A_{t}=\left|A_{t}\right| \mathrm{e}^{i \phi_{A_{t}}}$. On top, $\mu$ enters as another free parameter related to the Higgsino sector. These parameters have to be renormalized at the one-loop level,

$$
\begin{aligned}
m_{t} & \rightarrow m_{t}+\delta^{(1)} m_{t}, \\
\mathbf{M}_{\tilde{t}} & \rightarrow \mathbf{M}_{\tilde{t}}+\delta^{(1)} \mathbf{M}_{\tilde{t}}
\end{aligned}
$$

The independent renormalization conditions for the colored sector are formulated in the following way:

- The mass of the top quark is defined on-shell, i.e. ${ }^{2}$

$$
\delta^{(1)} m_{t}=m_{t} \tilde{\Re \mathfrak{e}}\left[\frac{1}{2}\left(\Sigma_{t}^{(1), \mathrm{L}}\left(m_{t}^{2}\right)+\Sigma_{t}^{(1), \mathrm{R}}\left(m_{t}^{2}\right)\right)+\Sigma_{t}^{(1), \mathrm{S}}\left(m_{t}^{2}\right)\right],
$$

according to the Lorentz decomposition of the self-energy of the top quark (the contributing Feynman diagrams are depicted in figure 4)

$$
\Sigma_{t}(p)=\not p \omega_{-} \Sigma_{t}^{\mathrm{L}}\left(p^{2}\right)+\not p \omega_{+} \Sigma_{t}^{\mathrm{R}}\left(p^{2}\right)+m_{t} \Sigma_{t}^{\mathrm{S}}\left(p^{2}\right)+m_{t} \gamma_{5} \Sigma_{t}^{\mathrm{PS}}\left(p^{2}\right) .
$$

- $m_{\tilde{q}_{3}}^{2}$ and $m_{\tilde{t}_{\mathrm{R}}}^{2}$ are traded for $m_{\tilde{t}_{1}}^{2}$ and $m_{\tilde{t}_{2}}^{2}$, which are then fixed by on-shell conditions for the top-squarks,

$$
\delta^{(1)} m_{\tilde{t}_{i}}^{2}=\tilde{\Re} \mathfrak{e}\left[\Sigma_{\tilde{t}_{i i}}^{(1)}\left(m_{\tilde{t}_{i}}^{2}\right)\right], \quad i=1,2,
$$

\footnotetext{
${ }^{2} \tilde{\mathfrak{R} e}$ denotes the real part of all loop integrals, but leaves the couplings unaffected.
} 
involving the diagonal $\tilde{t}_{1}$ and $\tilde{t}_{2}$ self-energies (diagrammatically visualized in figure 4 ). These on-shell conditions determine the diagonal entries of the counterterm matrix

$$
\mathbf{U}_{\tilde{t}} \delta \mathbf{M}_{\tilde{t}} \mathbf{U}_{\tilde{t}}^{\dagger}=\left(\begin{array}{cc}
\delta^{(1)} m_{\tilde{t}_{1}}^{2} & \delta^{(1)} m_{\tilde{t}_{1} \tilde{t}_{2}}^{2} \\
\delta^{(1)} m_{\tilde{t}_{1} \tilde{t}_{2}}^{2 *} & \delta^{(1)} m_{\tilde{t}_{2}}^{2}
\end{array}\right) .
$$

- The mixing parameter $A_{t}$ is correlated with the $\tilde{t}$-mass eigenvalues, $t_{\beta}$, and $\mu$, through eq. (3.2). Exploiting eq. (3.7) and the unitarity of $\mathbf{U}_{\tilde{t}}$ yields the expression

$$
\begin{aligned}
& \left(A_{t}-\frac{\mu^{*}}{t_{\beta}}\right) \delta^{(1)} m_{t}+m_{t}\left(\delta^{(1)} A_{t}-\frac{\delta^{(1)} \mu^{*}}{t_{\beta}}+\frac{\mu^{*} \delta^{(1)} t_{\beta}}{t_{\beta}^{2}}\right)= \\
& \mathbf{U}_{\tilde{t} 11} \mathbf{U}_{\tilde{t} 12}^{*}\left(\delta^{(1)} m_{\tilde{t}_{1}}^{2}-\delta^{(1)} m_{\tilde{t}_{2}}^{2}\right)+\mathbf{U}_{\tilde{t} 21} \mathbf{U}_{\tilde{t} 12}^{*} \delta^{(1)} m_{\tilde{t}_{1} \tilde{t}_{2}}^{2}+\mathbf{U}_{\tilde{t} 22} \mathbf{U}_{\tilde{t} 11}^{*} \delta^{(1)} m_{\tilde{t}_{1} \tilde{t}_{2}}^{2 *}
\end{aligned}
$$

For the non-diagonal entry of eq. (3.7), the renormalization condition

$$
\delta^{(1)} m_{\tilde{t}_{1} \tilde{t}_{2}}^{2}=\frac{1}{2} \tilde{\Re} \mathfrak{R}\left[\Sigma_{\tilde{t}_{12}}^{(1)}\left(m_{\tilde{t}_{1}}^{2}\right)+\Sigma_{\tilde{t}_{12}}^{(1)}\left(m_{\tilde{t}_{2}}^{2}\right)\right]
$$

is imposed, as in ref. [46], which involves the non-diagonal $\tilde{t}_{1}-\tilde{t}_{2}$ self-energy (figure 4). By means of eq. (3.8) the counterterm $\delta^{(1)} A_{t}$ is then determined. Actually this yields two conditions, for $\left|A_{t}\right|$ and for the phase $\phi_{A_{t}}$ separately. The additionally required mass counterterm $\delta^{(1)} \mu$ is obtained as described below in section 3.2.

- As already mentioned, the relevant sbottom mass is not an independent parameter, and hence its counterterm is a derived quantity that can be obtained from eq. (3.7),

$$
\begin{aligned}
\delta^{(1)} m_{\tilde{b}_{1}}^{2} \equiv \delta^{(1)} m_{\tilde{q}_{3}}^{2}= & \left|\mathbf{U}_{\tilde{t} 11}\right|^{2} \delta^{(1)} m_{\tilde{t}_{1}}^{2}+\left|\mathbf{U}_{\tilde{t} 12}\right|^{2} \delta^{(1)} m_{\tilde{t}_{2}}^{2} \\
& -\mathbf{U}_{\tilde{t} 22} \mathbf{U}_{\tilde{t} 12}^{*} \delta^{(1)} m_{\tilde{t}_{1} \tilde{t}_{2}}^{2}-\mathbf{U}_{\tilde{t} 12} \mathbf{U}_{\tilde{t} 22}^{*} \delta^{(1)} m_{\tilde{t}_{1} \tilde{t}_{2}}^{2}-2 m_{t} \delta^{(1)} m_{t} .
\end{aligned}
$$

\subsection{The chargino-neutralino sector}

For the calculation of the $\mathcal{O}\left(\alpha_{t}^{2}\right)$ contributions to the Higgs-boson self-energies and tadpoles, also the neutralino and chargino sectors have to be considered. Chargino/neutralino vertices and propagators enter only at the two-loop level and thus do not need renormalization; in the one-loop terms, however, the Higgsino-mass parameter $\mu$ enters via the couplings of Higgs bosons to stops and the counterterm $\delta^{(1)} \mu$ is required for the one-loop subrenormalization. The mass matrices in the bino/wino/higgsino bases are given by

$$
\mathbf{Y}=\left(\begin{array}{cccc}
M_{1} & 0 & -M_{Z} s_{\mathrm{w}} c_{\beta} & M_{Z} s_{\mathrm{w}} s_{\beta} \\
0 & M_{2} & M_{Z} c_{\mathrm{w}} c_{\beta} & M_{Z} c_{\mathrm{w}} s_{\beta} \\
-M_{Z} s_{\mathrm{w}} c_{\beta} & M_{Z} c_{\mathrm{w}} c_{\beta} & 0 & -\mu \\
M_{Z} s_{\mathrm{w}} s_{\beta} & M_{Z} c_{\mathrm{w}} s_{\beta} & -\mu & 0
\end{array}\right), \quad \mathbf{X}=\left(\begin{array}{cc}
M_{2} & \sqrt{2} M_{W} s_{\beta} \\
\sqrt{2} M_{W} c_{\beta} & \mu
\end{array}\right) .
$$




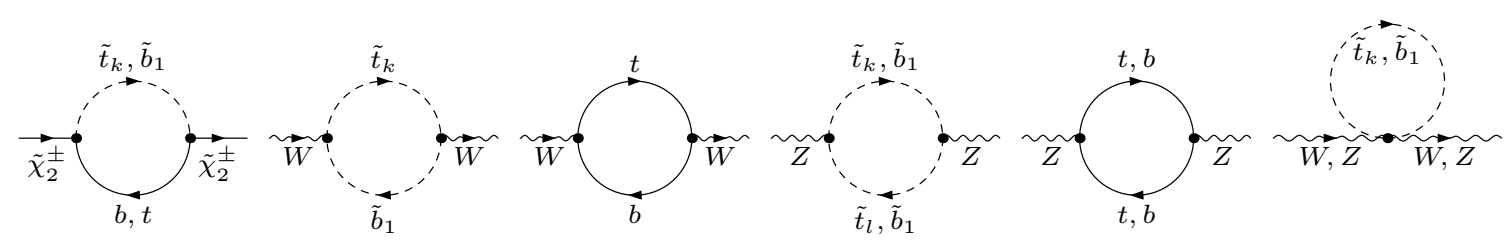

Figure 5. Feynman diagrams for the counterterms $\delta^{(1)} \mu, \delta^{(1)} M_{W}^{2} / M_{W}^{2}$, and $\delta^{(1)} M_{Z}^{2} / M_{Z}^{2}$.

Diagonal matrices with real and positive entries are obtained with the help of unitary matrices $\mathbf{N}, \mathbf{U}, \mathbf{V}$ by the singular value decompositions

$$
\mathbf{N}^{*} \mathbf{Y} \mathbf{N}^{\dagger}=\operatorname{diag}\left(m_{\tilde{\chi}_{1}^{0}}, m_{\tilde{\chi}_{2}^{0}}, m_{\tilde{\chi}_{3}^{0}}, m_{\tilde{\chi}_{4}^{0}}\right), \quad \mathbf{U}^{*} \mathbf{X} \mathbf{V}^{\dagger}=\operatorname{diag}\left(m_{\tilde{\chi}_{1}^{ \pm}}, m_{\tilde{\chi}_{2}^{ \pm}}\right) .
$$

In the gaugeless limit the off-diagonal $(2 \times 2)$-blocks of $\mathbf{Y}$ and the off-diagonal entries of $\mathbf{X}$ vanish. For this special case the transformation matrices and diagonal entries in eq. (3.12) simplify,

$$
\begin{aligned}
& \mathbf{N}=\left(\begin{array}{ccc}
\mathrm{e}^{\frac{i}{2} \phi_{M_{1}}} & 0 & \mathbf{0} \\
0 & \mathrm{e}^{\frac{i}{2} \phi_{M_{2}}} & \\
\mathbf{0} & \frac{1}{\sqrt{2}} \mathrm{e}^{\frac{i}{2} \phi_{\mu}}\left(\begin{array}{cc}
1 & -1 \\
i & i
\end{array}\right)
\end{array}\right), \quad \mathbf{U}=\left(\begin{array}{cc}
\mathrm{e}^{i \phi_{M_{2}}} & 0 \\
0 & \mathrm{e}^{i \phi_{\mu}}
\end{array}\right), \quad \mathbf{V}=\mathbf{1} ; \\
& m_{\tilde{\chi}_{1}^{0}}=\left|M_{1}\right|, \quad m_{\tilde{\chi}_{2}^{0}}=\left|M_{2}\right|, \quad m_{\tilde{\chi}_{3}^{0}}=|\mu|, \quad m_{\tilde{\chi}_{4}^{0}}=|\mu|, \quad m_{\tilde{\chi}_{1}^{ \pm}}=\left|M_{2}\right|, \quad m_{\tilde{\chi}_{2}^{ \pm}}=|\mu| ;
\end{aligned}
$$

and only the Higgsinos $\tilde{\chi}_{3}^{0}, \tilde{\chi}_{4}^{0}, \tilde{\chi}_{2}^{ \pm}$remain in the $\mathcal{O}\left(\alpha_{t}^{2}\right)$ contributions.

The Higgsino mass parameter $\mu$ is an independent input quantity and has to be renormalized accordingly, $\mu \rightarrow \mu+\delta^{(1)} \mu$, fixing the counterterm $\delta^{(1)} \mu$ by an independent renormalization condition, which renders the one-loop subrenormalization complete. Together with the soft-breaking parameters $M_{1}$ and $M_{2}, \mu$ can be defined in the neutralino/chargino sector by requiring on-shell conditions for the two charginos and one neutralino.

However, since only $\delta^{(1)} \mu$ is required here, it is sufficient to impose a renormalization condition for $\tilde{\chi}_{2}^{ \pm}$only; the appropriate on-shell condition reads,

$$
\begin{aligned}
\delta^{(1)} \mu & =\mathrm{e}^{i \phi_{\mu}} \delta^{(1)}|\mu|, \\
\delta^{(1)}|\mu| & =|\mu|\left\{\frac{1}{2}\left[\Re \mathfrak{R}\left[\Sigma_{\tilde{\chi}_{2}^{ \pm}}^{(1), \mathrm{L}}\left(|\mu|^{2}\right)+\Sigma_{\tilde{\chi}_{2}^{ \pm}}^{(1), \mathrm{R}}\left(|\mu|^{2}\right)\right]\right]+\Re \mathfrak{R}\left[\Sigma_{\tilde{\chi}_{2}^{ \pm}}^{(1), \mathrm{S}}\left(|\mu|^{2}\right)\right]\right\},
\end{aligned}
$$

where the Lorentz decomposition of the self-energy for the Higgsino-like chargino $\tilde{\chi}_{2}^{ \pm}$(see figure 5) has been applied, in analogy to eq. (3.5).

Another option is the $\overline{\mathrm{DR}}$-renormalization of $\mu$, which defines the counterterm $\delta^{(1)} \mu$ in the $\overline{\mathrm{DR}}$-scheme, i.e. by the divergent part of the expression in eq. (3.14).

\section{Numerical results for masses and mixings}

In this section we present numerical analyses for the masses of the neutral Higgs bosons derived from eq. (2.14) in various SUSY-parameter scenarios. The complete one-loop results 


\begin{tabular}{|cc|}
\hline MSSM input & SM input \\
\hline$M_{2}=200 \mathrm{GeV}$, & $m_{t}=173.2 \mathrm{GeV}$, \\
$M_{1}=\left(5 s_{\mathrm{w}}^{2}\right) /\left(3 c_{\mathrm{w}}^{2}\right) M_{2}$, & $m_{b}=4.2 \mathrm{GeV}$, \\
$m_{\tilde{l}_{1}}=m_{\tilde{e}_{\mathrm{R}}}=2000 \mathrm{GeV}$, & $m_{\tau}=1.77703 \mathrm{GeV}$, \\
$m_{\tilde{q}_{1}}=m_{\tilde{u}_{\mathrm{R}}}=m_{\tilde{d}_{\mathrm{R}}}=2000 \mathrm{GeV}$, & $M_{W}=80.385 \mathrm{GeV}$, \\
$A_{u}=A_{d}=A_{e}=0 \mathrm{GeV}$, & $M_{Z}=91.1876 \mathrm{GeV}$, \\
$m_{\tilde{l}_{2}}=m_{\tilde{\mu}_{\mathrm{R}}}=2000 \mathrm{GeV}$, & $G_{\mathrm{F}}=1.16639 \cdot 10^{-5}$, \\
$m_{\tilde{q}_{2}}=m_{\tilde{c}_{\mathrm{R}}}=m_{\tilde{s}_{\mathrm{R}}}=2000 \mathrm{GeV}$, & $\alpha_{s}\left(M_{Z}\right)=0.118$, \\
$A_{c}=A_{s}=A_{\mu}=0 \mathrm{GeV}$, & $1 / \alpha=128.945$. \\
\hline
\end{tabular}

Table 1. Default input values of the MSSM and SM parameters.

with the full dependence on the external squared-momentum $p^{2}$, and the two-loop $\mathcal{O}\left(\alpha_{t} \alpha_{s}\right)$ terms are taken from FeynHiggs, while the $\mathcal{O}\left(\alpha_{t}^{2}\right)$ terms are computed by means of the corresponding two-loop self-energies as specified in the previous sections. In our strategy, the new $\mathcal{O}\left(\alpha_{t}^{2}\right)$ self-energies are combined with the complementary self-energies according to eq. (2.13) within FeynHiggs, and the masses are then derived via eq. (2.14), ordered as $m_{h_{1}}<m_{h_{2}}<m_{h_{3}}$.

The Standard Model (SM) parameters are collected in table 1, as well as those MSSM parameters that are kept for the analyses which are performed in this section. The residual input parameters of the MSSM are shown in the figures or their captions. The parameters $\mu, t_{\beta}$, and the Higgs field-renormalization constants are defined in the $\overline{\mathrm{DR}}$ scheme at the scale $m_{t}$.

Higgs-boson masses in the real MSSM. In the case of the MSSM with real parameters, conventionally the mass $m_{A}$ of the $C P$-odd $A$ boson is chosen as an input parameter, and the masses of the two $C P$-even neutral scalar bosons are predicted in terms of $m_{A}$ and the other SUSY parameters. In this special case, a comparison of our diagrammatic result with those of the previously known $\mathcal{O}\left(\alpha_{t}^{2}\right)$ contributions [28] obtained by the effectivepotential method is possible. In practice, this comparison is made by means of the default version of FeynHiggs which incorporates the $\mathcal{O}\left(\alpha_{t}^{2}\right)$ terms from [28]. The beautiful agreement between the two independent calculations has been shown recently for the mass of the lightest Higgs boson in ref. [50]; similar good agreement has been found also for the heavier Higgs-boson mass. The impact of the $\mathcal{O}\left(\alpha_{t}^{2}\right)$ terms in particular on the mass of the lightest Higgs boson is substantial, yielding a mass shift of $\approx 5 \mathrm{GeV}$, and demonstrates the importance of the two-loop Yukawa contributions for a reliable prediction of the Higgsboson masses. For complex parameters, additional mass shifts of several $\mathrm{GeV}$ can occur from the complex phases.

Higgs-boson masses in the complex MSSM. In the current public version of FeynHiggs for complex parameters, the dependence of the $\mathcal{O}\left(\alpha_{t}^{2}\right)$ terms on the phases of $\phi_{A_{t}}$ and $\phi_{\mu}$ is approximated by an interpolation between the real results for the phases 0 


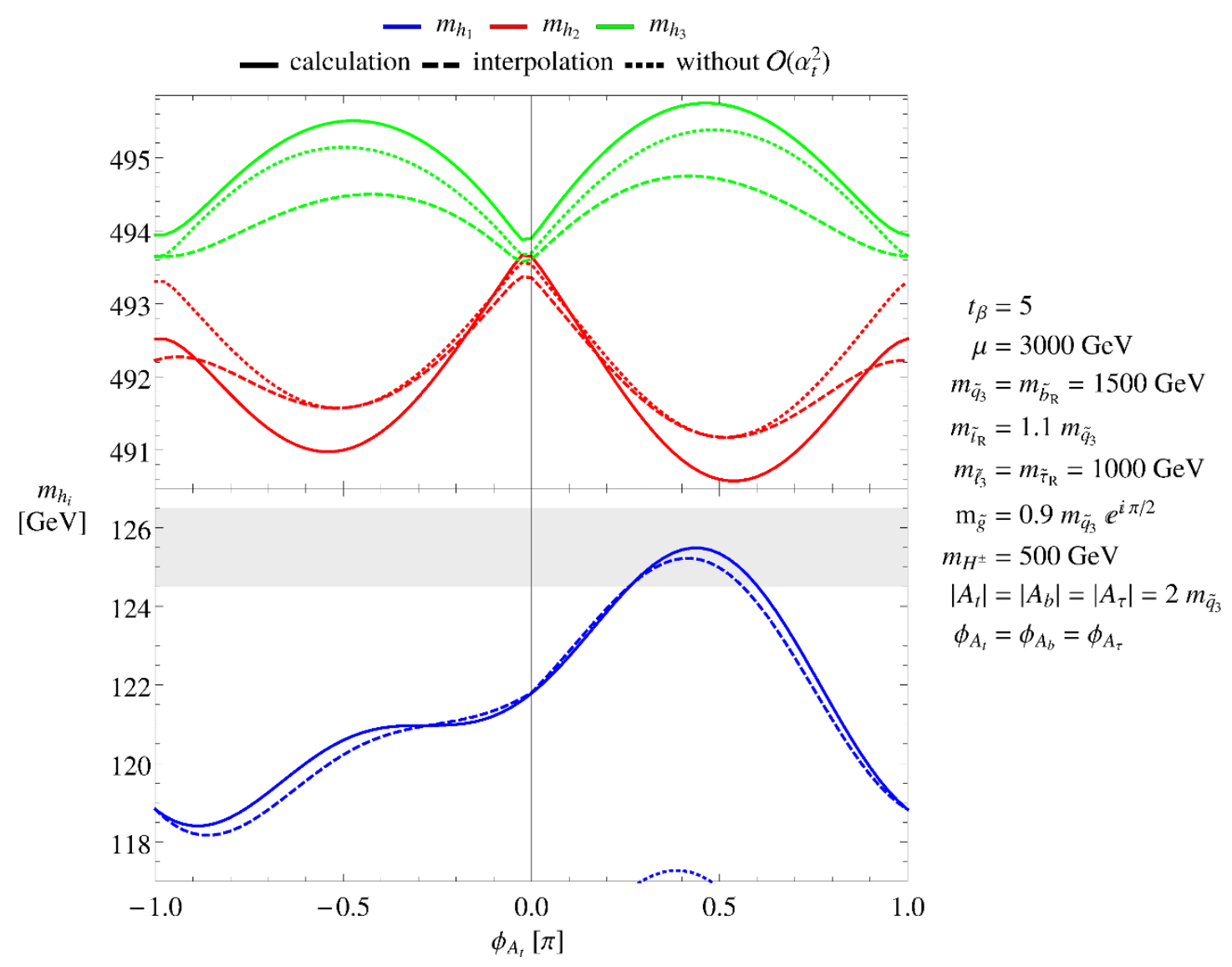

Figure 6. The result for the masses of the neutral Higgs bosons from the diagrammatic calculation (full), in comparison with the approximate result from interpolation between the phases $\phi_{A_{t}}=0, \pm \pi$ (dashed). For reference the result without the $\mathcal{O}\left(\alpha_{t}^{2}\right)$ contributions is depicted (dotted). The gray area depicts the mass range between $124.5 \mathrm{GeV}$ and $126.5 \mathrm{GeV}$.

and $\pm \pi[63,64]$. In ref. [50] a comparison with the full diagrammatic calculation for the mass of the lightest Higgs boson was presented showing notable deviations. Figure 6 contains the comparison for all three mass eigenvalues. The dependence of the heavier (upper plot) and the lightest (lower plot) neutral Higgs-boson masses on the phase $\phi_{A_{t}}$ is illustrated. Rather large deviations from the previous result of FeynHiggs are found owing to the $\mathcal{O}\left(\alpha_{t}^{2}\right)$ contributions to the charged-Higgs self-energy which were not known before but which are required for consistent renormalization of the self-energies of the neutral Higgs bosons in the complex MSSM. So far, FeynHiggs utilized the known $\mathcal{O}\left(\alpha_{t}^{2}\right)$ contribution to the self-energy of the $A$-boson for renormalization; the visible deviations at the real edges in figure 6 originate from the difference of these renormalization schemes, i.e. the $\mathcal{O}\left(\alpha_{t}^{2}\right)$ terms in the difference $\Sigma_{A}-\Sigma_{H^{ \pm}}$. A similar effect has also been found previously in the $\mathcal{O}\left(\alpha_{t} \alpha_{s}\right)$ corrections to the $m_{A}-m_{H^{ \pm}}$mass correlation in ref. [65].

$\boldsymbol{C P}$-mixing. In the complex MSSM all three neutral Higgs bosons mix at higher orders according to the off-diagonal entries of the mass matrix in eq. (2.14), leading to violation 

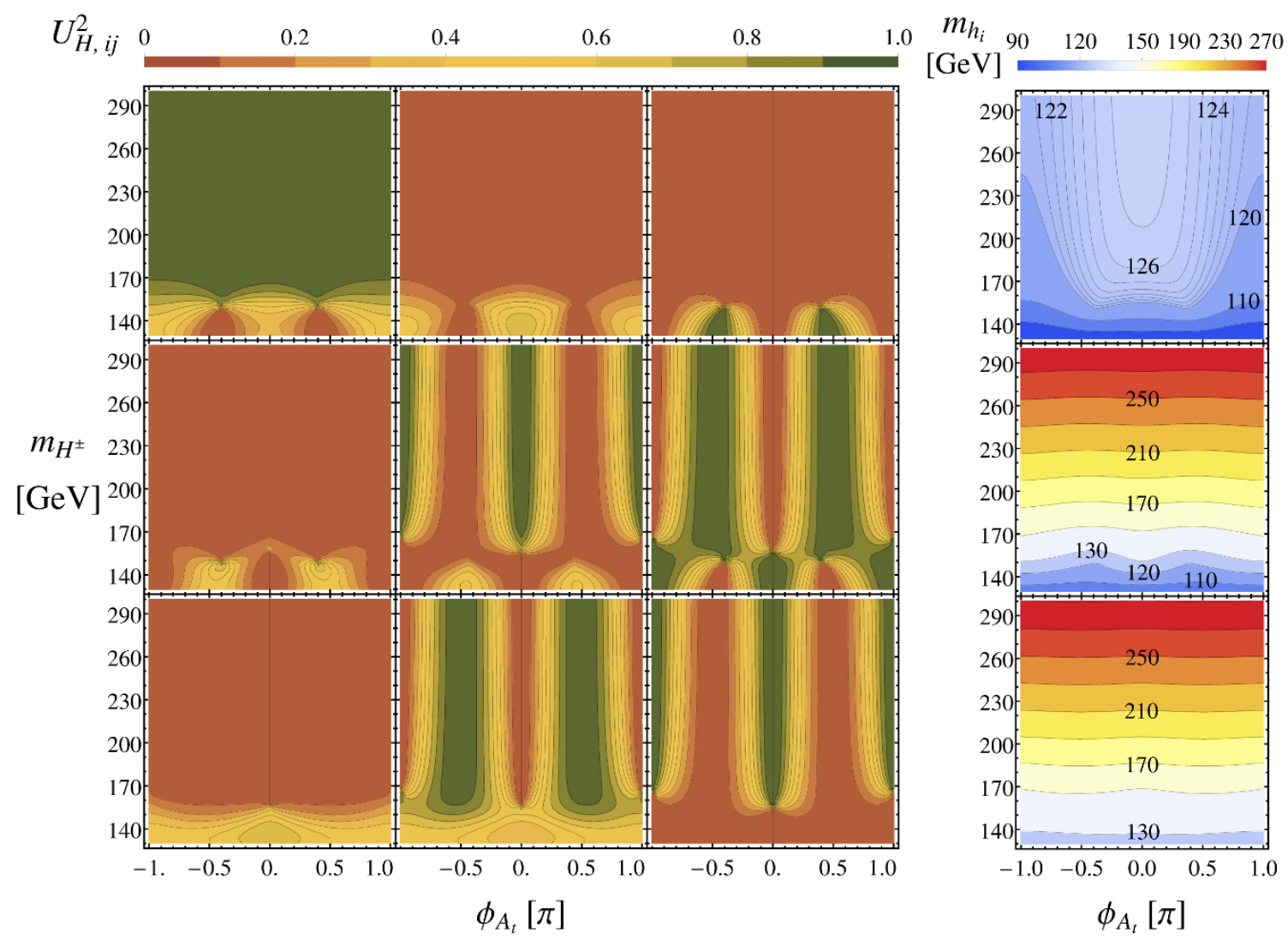

Figure 7. The mixing-matrix elements squared $U_{H, i j}^{2}, i, j \in\{1,2,3\}$ (left) and masses $m_{h_{i}}$ (right), where $i$ is the index of the row and $j$ is the index of the column, are illustrated with the phase $\phi_{A_{t}}$ and the input mass $m_{H^{ \pm}}$being varied. The color coding is explained in the labels at the top of the figures; for convenience some contours of the mass plots are signed with their corresponding mass values. The input parameters are fixed at $\mu=2500 \mathrm{GeV}, t_{\beta}=10, m_{\tilde{\ell}_{3}}=m_{\tilde{\tau}_{\mathrm{R}}}=1500 \mathrm{GeV}$, $m_{\tilde{q}_{3}}=m_{\tilde{t}_{\mathrm{R}}}=m_{\tilde{b}_{\mathrm{R}}}=1500 \mathrm{GeV},\left|A_{t}\right|=\left|A_{b}\right|=\left|A_{\tau}\right|=2 m_{\tilde{q}_{3}}, m_{\tilde{g}}=2000 \mathrm{GeV}$.

of $C P$-symmetry. Since the self-energies contributing to the mass matrix at higher orders are momentum-dependent, this $C P$-mixing depends on $p^{2}$ and hence it is not possible to describe $C P$-mixing in terms of a constant mixing matrix. A convenient approximation for the discussion of $C P$-mixing is given by setting $p^{2}=0$ in the renormalized self-energies of the Higgs bosons at all orders. In this case, eq. (2.14) simplifies to the eigenvalue equation for the matrix $\mathbf{M}_{h H A}^{(2)}(0)$ in eq. (2.13). The real mixing matrix which diagonalizes $\mathbf{M}_{h H A}^{(2)}(0)$ is denoted by $\mathbf{U}_{H}$ in the following. It allows to define an approximate mass-eigenstate basis according to

$$
\left(\begin{array}{l}
h_{1} \\
h_{2} \\
h_{3}
\end{array}\right)=\mathbf{U}_{H}\left(\begin{array}{c}
h \\
H \\
A
\end{array}\right), \quad \mathbf{U}_{H}=\left(\begin{array}{lll}
U_{H, 11} & U_{H, 12} & U_{H, 13} \\
U_{H, 21} & U_{H, 22} & U_{H, 23} \\
U_{H, 31} & U_{H, 32} & U_{H, 33}
\end{array}\right) .
$$

In general the $h_{i}, i \in\{1,2,3\}$, are no longer $C P$-eigenstates since they are composed of the $C P$-even $h$ and $H$ and the $C P$-odd $A$. The elements $U_{H, i 3}$ of $\mathbf{U}_{H}$ in eq. (4.1) squared 
tell the amount of the $A$ boson inside of

$$
h_{i}=U_{H, i 1} h+U_{H, i 2} H+U_{H, i 3} A
$$

and thus the $C P$-odd admixture in $h_{i}$.

The dependence of the mixing-matrix elements squared $U_{H, i j}^{2}$, in the approximation of eq. (4.1), on the charged Higgs-boson mass $m_{H^{ \pm}}$and the basically unconstrained complex phase $\phi_{A_{t}}[66-74]$ is shown in the left part of figure 7 . Therein, the tiles are ordered according to the matrix array of $U_{H, i j}^{2}$, with $i$ and $j$ indicating row and column, respectively. The right part of figure 7 displays the masses $m_{h_{i}}$ in ascending order from the first to the third row.

Whenever two masses $m_{h_{i}}^{2}$ and $m_{h_{j}}^{2}$ are close to each other, the entries $U_{H, i j}^{2}$ rapidly change from zero to unity, i.e. $h_{i}$ and $h_{j}$ interchange their meaning. For a large value of the charged Higgs-boson mass, the lightest Higgs $h_{1}$ is basically equal to $h$. In contrast, the heavier Higgs bosons can be composed of $H$ and $A$ in all possible variations, depending on the phase, thus yielding the possibility of very large $C P$-mixing. At the nodal points at the real values of $A_{t}$ for $\phi_{A_{t}}= \pm \pi$ and $m_{H^{ \pm}} \approx 165 \mathrm{GeV}$ as well as for $\phi_{A_{t}}=0$ and $m_{H^{ \pm}} \approx 155 \mathrm{GeV}$ the masses of $h_{2}$ and $h_{3}$ are equal; slightly above or below the nodes, $h_{2}$ and $h_{3}$ can be identified as $H$ or $A$. Between $m_{H^{ \pm}} \approx 155 \mathrm{GeV}$ and $m_{H^{ \pm}} \approx 150 \mathrm{GeV}$ an extreme situation is observed, where each $h_{i}$ is almost equal to $h, H$ or $A$ for any complex phase. However the situation changes for lower input values of $m_{H^{ \pm}}$ where a large admixture of $A$ to the lightest Higgs boson $h_{1}$ is predicted, depending on the complex phase $\phi_{A_{t}}$. In the same parameter range the heaviest state $h_{3}$ is nearly $C P$-even for any phase. In this scenario the Higgs-like particle discovered at the LHC is interpreted as the heaviest neutral Higgs boson of the MSSM (so-called low- $m_{H}$ scenario). The strongest gradients for the mixing of $h$ and $A$ to $h_{1}$ and $h_{2}$ appear at $\phi_{A_{t}} \approx \pm 0.4$ and $m_{H^{ \pm}} \approx 150 \mathrm{GeV}$; for the present choice of parameters the masses $m_{h_{1}}$ and $m_{h_{2}}$ are equal at this point.

For the same parameter set, in figure 8 the Higgs-boson masses are depicted for the two special cases of $m_{H^{ \pm}}=170 \mathrm{GeV}$ (left) and $m_{H^{ \pm}}=250 \mathrm{GeV}$ (right). For larger $m_{H^{ \pm}}$the mass of the lightest Higgs boson can be lowered by an increasing phase $\phi_{A_{t}}$ to be in the mass range of the experimentally discovered Higgs-like particle, remaining essentially $C P$-even, whereas the heavier Higgs bosons get a larger mass splitting and a substantial $C P$-mixing. For the lower $m_{H^{ \pm}}$value, the phase cannot be too large for the right mass $m_{h_{1}}$, and mass splitting of the two heavy Higgs bosons shows a stronger variation.

Complex-valued $\boldsymbol{\mu}$. Also the coefficient $\mu$ of the bilinear term of the superpotential is in general a complex quantity. The phase of $\mu$ is severely constrained by the experimental limits on the electric dipole moments of electron and neutron. These bounds can, however, be circumvented in principle by a specific fine-tuning of the phases of $\mu$ and of the nonuniversal SUSY parameters [71, 73, 75-77], leaving room also for a non-vanishing phase $\phi_{\mu}$, and thus we want to illustrate potential effects of $\phi_{\mu} \neq 0$ in terms of an example. In figure 9 we display the influence of the phases $\phi_{\mu}$ and $\phi_{X_{t}}$ (with $X_{t}=A_{t}^{*}-\mu / t_{\beta}$ ) on the mass of the lightest Higgs boson. In the depicted scenario the variation of $m_{h_{1}}$ with $\phi_{\mu}$ is of the order of $0.5 \mathrm{GeV}$. Changing of the sign of $\phi_{\mu}$ mirrors the graphs at the axis $\phi_{X_{t}}=0$. 

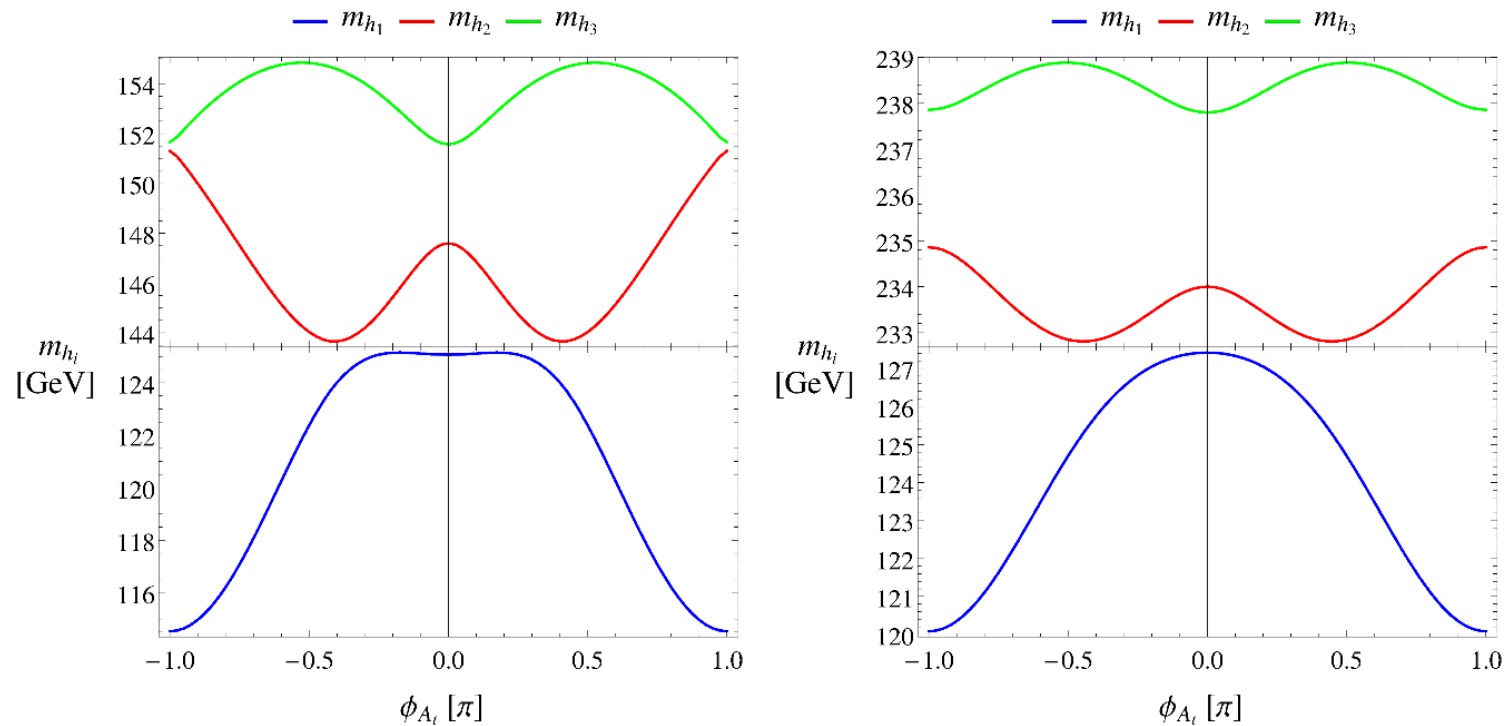

Figure 8. The dependence of the neutral Higgs-boson masses on the phase $\phi_{A_{t}}$ is depicted for an input value of the charged Higgs-boson mass of $m_{H^{ \pm}}=170 \mathrm{GeV}$ (left) and $m_{H^{ \pm}}=250 \mathrm{GeV}$ (right). The other input parameters are the same as in figure 7 .
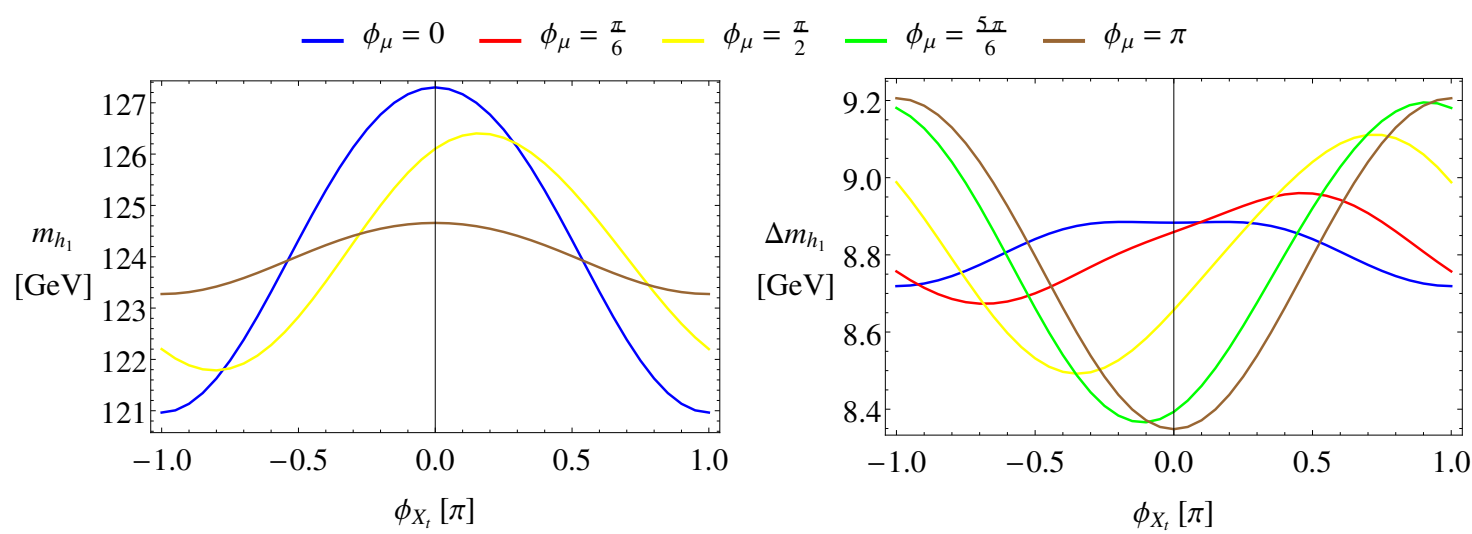

Figure 9. The dependence of the lightest neutral Higgs-boson mass on the phases $\phi_{X_{t}}$ and $\phi_{\mu}$ is depicted. Left: the value of $m_{h_{1}}$ including all available contributions, with the phase dependence arising from one-loop, $\mathcal{O}\left(\alpha_{t} \alpha_{s}\right)$ and $\mathcal{O}\left(\alpha_{t}^{2}\right)$ terms. Right: the contribution $\Delta m_{h_{1}}$ to $m_{h_{1}}$ owing exclusively to the $\mathcal{O}\left(\alpha_{t}^{2}\right)$ terms, for different phases. The input parameters are $m_{H^{ \pm}}=200 \mathrm{GeV}$, $|\mu|=2500 \mathrm{GeV}, t_{\beta}=10, m_{\tilde{\ell}_{3}}=m_{\tilde{\tau}_{\mathrm{R}}}=1000 \mathrm{GeV}, m_{\tilde{q}_{3}}=m_{\tilde{t}_{\mathrm{R}}}=m_{\tilde{b}_{\mathrm{R}}}=1500 \mathrm{GeV}, \quad\left|X_{t}\right|=2 m_{\tilde{q}_{3}}$, $A_{b}=A_{\tau}=0, m_{\tilde{g}}=2000 \mathrm{GeV}$. 
As one can see in the expressions in appendix D, the off-diagonal self-energies $\Sigma_{h A}^{(2)}$ and $\Sigma_{H A}^{(2)}$ are proportional to $\Im \mathfrak{m}\left[X_{t} \mu^{*}\right]$; thus no $C P$-mixing by the $\mathcal{O}\left(\alpha_{t}^{2}\right)$ terms occurs for $\phi_{X_{t}}-\phi_{\mu}=0, \pm \pi$. Nevertheless, large mass shifts do occur.

\section{Conclusions}

We have presented the full results for the leading two-loop Yukawa contributions of $\mathcal{O}\left(\alpha_{t}^{2}\right)$ from the top-stop sector in the calculation of the Higgs-boson masses of the MSSM with complex parameters. They generalize the previously known result for the real MSSM to the case of complex phases entering at the two-loop level. The combination of the new terms with the hitherto available full one-loop result and leading two-loop terms of $\mathcal{O}\left(\alpha_{t} \alpha_{s}\right)$ yields an improved prediction for the Higgs-boson mass spectrum also for complex parameters that is equivalent in accuracy to that of the real MSSM.

In the complex MSSM, the masses of the three neutral Higgs are derived quantities whereas the mass of the charged Higgs boson is chosen as an input parameter. The mass shifts that originate from the $\mathcal{O}\left(\alpha_{t}^{2}\right)$ terms are significant, and hence an adequate treatment also for complex parameters is a necessity. Besides the mass shift of about $5 \mathrm{GeV}$ in the real MSSM, additional shifts of the same size can be induced by complex parameters.

Large $C P$-mixing among the heavy Higgs bosons is found for $m_{H^{ \pm}} \gtrsim 160 \mathrm{GeV}$. In this case the lightest Higgs boson is basically $C P$-even and can be identified with the Higgs signal observed in the LHC experiments ATLAS and CMS. At lower values of the charged Higgs-boson mass, the lighter Higgs bosons can be strongly $C P$-mixed, with low masses, and with the heaviest Higgs boson basically $C P$-even. In this case, the observed Higgs particle can be interpreted as the heaviest neutral Higgs boson of the MSSM. Such a scenario, however, might be ruled out by the experimental exclusion of a light charged Higgs particle.

Our new results will become part of the code FeynHiggs, where so far the complex phases are treated in an approximate way by interpolating between the real results for phases 0 and $\pm \pi$. At the formal side, we have given the complete counterterm structure at the two-loop level for the renormalization of the self-energies in the Higgs sector of the complex MSSM, which can be used for further two-loop calculations going beyond the Yukawa approximation.

\section{Acknowledgments}

We thank Stefano Di Vita, Thomas Hahn, Sven Heinemeyer, Heidi Rzehak, Pietro Slavich, Dominik Stöckinger, Alexander Voigt, and Georg Weiglein for helpful discussions.

\section{A Two-loop mass counterterms}

The genuine two-loop mass counterterms introduced in eq. (2.16b) and eq. (2.17b) are listed in the following. Thereby $\delta^{(1)} e, \delta^{(1)} M_{W}$ and $\delta^{(1)} s_{\mathrm{w}}$ always appear in the combina- 
tion $\delta^{(1)} Z_{\mathrm{w}}=\delta^{(1)} e / e-\delta^{(1)} M_{W} / M_{W}-\delta^{(1)} s_{\mathrm{w}} / s_{\mathrm{w}}$ :

$$
\begin{aligned}
& \delta^{(2)} m_{h}^{2}=c_{\alpha-\beta}^{2} \delta^{(2)} m_{A}^{2}+s_{\alpha+\beta}^{2} \delta^{(2)} m_{Z}^{2}+c_{\beta}^{2} \delta^{(2)} t_{\beta}\left(s_{2(\alpha-\beta)} m_{A}^{2}+s_{2(\alpha+\beta)} m_{Z}^{2}\right) \\
& +c_{\beta}^{2} \delta^{(1)} t_{\beta}\left(s_{2(\alpha-\beta)} \delta^{(1)} m_{A}^{2}+s_{2(\alpha+\beta)} \delta^{(1)} m_{Z}^{2}\right) \\
& +\frac{1}{2} c_{\beta}^{3}\left(\delta^{(1)} t_{\beta}\right)^{2}\left[s_{\alpha-\beta}\left(3 s_{\alpha-2 \beta}-s_{\alpha}\right) m_{A}^{2}+2 c_{2 \alpha+3 \beta} m_{Z}^{2}\right] \\
& +\frac{e s_{\alpha-\beta}}{2 M_{W} s_{\mathrm{w}}}\left[\left(1+c_{\alpha-\beta}^{2}\right)\left(\delta^{(2)} T_{h}+\delta^{(1)} T_{h} \delta^{(1)} Z_{\mathrm{w}}\right)\right. \\
& +s_{\alpha-\beta} c_{\alpha-\beta}\left(\delta^{(2)} T_{H}+\delta^{(1)} T_{H} \delta^{(1)} Z_{\mathrm{w}}\right) \\
& \left.+s_{\alpha-\beta} c_{\beta}^{2} \delta^{(1)} t_{\beta}\left(c_{\alpha-\beta} \delta^{(1)} T_{h}+s_{\alpha-\beta} \delta^{(1)} T_{H}\right)\right], \\
& \delta^{(2)} m_{H}^{2}=s_{\alpha-\beta}^{2} \delta^{(2)} m_{A}^{2}+c_{\alpha+\beta}^{2} \delta^{(2)} m_{Z}^{2}-c_{\beta}^{2} \delta^{(2)} t_{\beta}\left(s_{2(\alpha-\beta)} m_{A}^{2}+s_{2(\alpha+\beta)} m_{Z}^{2}\right) \\
& -c_{\beta}^{2} \delta^{(1)} t_{\beta}\left(s_{2(\alpha-\beta)} \delta^{(1)} m_{A}^{2}+s_{2(\alpha+\beta)} \delta^{(1)} m_{Z}^{2}\right) \\
& +\frac{1}{2} c_{\beta}^{3}\left(\delta^{(1)} t_{\beta}\right)^{2}\left[c_{\alpha-\beta}\left(3 c_{\alpha-2 \beta}-c_{\alpha}\right) m_{A}^{2}-2 c_{2 \alpha+3 \beta} m_{Z}^{2}\right] \\
& -\frac{e c_{\alpha-\beta}}{2 M_{W} s_{\mathrm{w}}}\left[\left(1+s_{\alpha-\beta}^{2}\right)\left(\delta^{(2)} T_{H}+\delta^{(1)} T_{H} \delta^{(1)} Z_{\mathrm{w}}\right)\right. \\
& +c_{\alpha-\beta} s_{\alpha-\beta}\left(\delta^{(2)} T_{h}+\delta^{(1)} T_{h} \delta^{(1)} Z_{\mathrm{w}}\right) \\
& \left.-c_{\alpha-\beta} c_{\beta}^{2} \delta^{(1)} t_{\beta}\left(c_{\alpha-\beta} \delta^{(1)} T_{h}+s_{\alpha-\beta} \delta^{(1)} T_{H}\right)\right], \\
& \delta^{(2)} m_{G}^{2}=c_{\beta}^{4} m_{A}^{2}\left(\delta^{(1)} t_{\beta}\right)^{2} \\
& +\frac{e}{2 M_{W} s_{\mathrm{w}}}\left[s_{\alpha-\beta}\left(\delta^{(2)} T_{h}+\delta^{(1)} T_{h} \delta^{(1)} Z_{\mathrm{w}}\right)\right. \\
& -c_{\alpha-\beta}\left(\delta^{(2)} T_{H}+\delta^{(1)} T_{H} \delta^{(1)} Z_{\mathrm{w}}\right) \\
& \left.+c_{\beta}^{2} \delta^{(1)} t_{\beta}\left(c_{\alpha-\beta} \delta^{(1)} T_{h}+s_{\alpha-\beta} \delta^{(1)} T_{H}\right)\right], \\
& \delta^{(2)} m_{h H}^{2}=c_{\alpha-\beta} s_{\alpha-\beta} \delta^{(2)} m_{A}^{2}-c_{\beta}^{2} \delta^{(2)} t_{\beta}\left(c_{2(\alpha-\beta)} m_{A}^{2}+c_{2(\alpha+\beta)} m_{Z}^{2}\right) \\
& -c_{\alpha+\beta} s_{\alpha+\beta} \delta^{(2)} m_{Z}^{2}-c_{\beta}^{2} \delta^{(1)} t_{\beta}\left(c_{2(\alpha-\beta)} \delta^{(1)} m_{A}^{2}+c_{2(\alpha+\beta)} \delta^{(1)} m_{Z}^{2}\right) \\
& +\frac{1}{2} c_{\beta}^{3}\left(\delta^{(1)} t_{\beta}\right)^{2}\left[\left(-3 c_{\beta} s_{2(\alpha-\beta)}+2 s_{2 \alpha-\beta}\right) m_{A}^{2}+2 s_{2 \alpha+3 \beta} m_{Z}^{2}\right] \\
& +\frac{e}{2 M_{W} s_{\mathrm{w}}}\left[-c_{\alpha-\beta}^{3}\left(\delta^{(2)} T_{h}+\delta^{(1)} T_{h} \delta^{(1)} Z_{\mathrm{w}}\right)\right. \\
& +s_{\alpha-\beta}^{3}\left(\delta^{(2)} T_{H}+\delta^{(1)} T_{H} \delta^{(1)} Z_{\mathrm{w}}\right) \\
& \left.-c_{\alpha-\beta} s_{\alpha-\beta} c_{\beta}^{2} \delta^{(1)} t_{\beta}\left(c_{\alpha-\beta} \delta^{(1)} T_{h}+s_{\alpha-\beta} \delta^{(1)} T_{H}\right)\right] \text {, }
\end{aligned}
$$




$$
\begin{aligned}
& \delta^{(2)} m_{h A}^{2}=\frac{e}{2 M_{W} s_{\mathrm{w}}} s_{\alpha-\beta}\left(\delta^{(2)} T_{A}+\delta^{(1)} T_{A} \delta^{(1)} Z_{\mathrm{w}}\right), \\
& \delta^{(2)} m_{h G}^{2}=\frac{e}{2 M_{W} s_{\mathrm{w}}} c_{\alpha-\beta}\left(\delta^{(2)} T_{A}+\delta^{(1)} T_{A} \delta^{(1)} Z_{\mathrm{w}}\right), \\
& \delta^{(2)} m_{H A}^{2}=-\delta^{(2)} m_{h G}^{2}, \\
& \delta^{(2)} m_{H G}^{2}=\delta^{(2)} m_{h A}^{2} \text {, } \\
& \delta^{(2)} m_{A G}^{2}=-c_{\beta}^{2} m_{A}^{2} \delta^{(2)} t_{\beta}-c_{\beta}^{2} \delta^{(1)} m_{A}^{2} \delta^{(1)} t_{\beta}+c_{\beta}^{3} s_{\beta} m_{A}^{2}\left(\delta^{(1)} t_{\beta}\right)^{2} \\
& -\frac{e}{2 M_{W} s_{\mathrm{w}}}\left[c_{\alpha-\beta}\left(\delta^{(2)} T_{h}+\delta^{(1)} T_{h} \delta^{(1)} Z_{\mathrm{w}}\right)\right. \\
& \left.+s_{\alpha-\beta}\left(\delta^{(2)} T_{H}+\delta^{(1)} T_{H} \delta^{(1)} Z_{\mathrm{w}}\right)\right], \\
& \delta^{(2)} m_{H^{ \pm}}^{2}=\delta^{(2)} m_{A}^{2}+\delta^{(2)} M_{W}^{2}, \\
& \delta^{(2)} m_{G^{ \pm}}^{2}=c_{\beta}^{4} m_{H^{ \pm}}^{2}\left(\delta^{(1)} t_{\beta}\right)^{2} \\
& +\frac{e}{2 M_{W} s_{\mathrm{w}}}\left[s_{\alpha-\beta}\left(\delta^{(2)} T_{h}+\delta^{(1)} T_{h} \delta^{(1)} Z_{\mathrm{w}}\right)\right. \\
& -c_{\alpha-\beta}\left(\delta^{(2)} T_{H}+\delta^{(1)} T_{H} \delta^{(1)} Z_{\mathrm{w}}\right) \\
& \left.+c_{\beta}^{2} \delta^{(1)} t_{\beta}\left(c_{\alpha-\beta} \delta^{(1)} T_{h}+s_{\alpha-\beta} \delta^{(1)} T_{H}\right)\right], \\
& \delta^{(2)} m_{H^{-} G^{+}}^{2}=-c_{\beta}^{2} m_{H^{ \pm}}^{2} \delta^{(2)} t_{\beta}+c_{\beta}^{3} s_{\beta} m_{H^{ \pm}}^{2}\left(\delta^{(1)} t_{\beta}\right)^{2} \\
& -\frac{e}{2 M_{W} s_{\mathrm{w}}}\left[c_{\alpha-\beta}\left(\delta^{(2)} T_{h}+\delta^{(1)} T_{h} \delta^{(1)} Z_{\mathrm{w}}\right)\right. \\
& +s_{\alpha-\beta}\left(\delta^{(2)} T_{H}+\delta^{(1)} T_{H} \delta^{(1)} Z_{\mathrm{w}}\right) \\
& \left.+i\left(\delta^{(2)} T_{A}+\delta^{(1)} T_{A} \delta^{(1)} Z_{\mathrm{w}}\right)\right], \\
& \delta^{(2)} m_{G^{-} H^{+}}^{2}=\left(\delta^{(2)} m_{H^{-} G^{+}}^{2}\right)^{*} .
\end{aligned}
$$

The neutral counterterms are symmetric, i.e. $\delta^{(2)} m_{a b}^{2}=\delta^{(2)} m_{b a}^{2} \quad(a, b=h, H, A, G)$.

\section{B Couplings and counterterm insertions}

\section{B.1 Tree-level vertices}

The tree-level vertices contain the top-Yukawa coupling $h_{t}=m_{t} / v_{2}$. In the case of the Higgs bosons, their different couplings are accommodated by explicit factors $c(\ldots)$. The symbols $\Phi^{0}$ and $\Phi^{ \pm}$are used as generic expressions for the Higgs bosons, i.e. $\Phi^{0} \in\{h, H, A, G\}$ and $\Phi^{ \pm} \in\left\{H^{ \pm}, G^{ \pm}\right\}$. For fermion couplings, the left-chiral part is the first and the right-chiral part the second entry of the column. The present approximations have already been applied to this expressions, leaving only those parts proportional 
to $h_{t}$ or $h_{t}^{2}$. The mixing matrix $\mathbf{U}$ of the charginos does not appear in the following; $\mathbf{V}=\mathbf{1}$ is already inserted.

$$
\begin{aligned}
= & -i C\left(\Phi_{i}^{0}, \Phi_{j}^{0}, \tilde{t}_{k}, \tilde{t}_{l}\right) \\
= & -i h_{t}^{2}\left[c_{4}\left(\Phi_{i}^{0}, \Phi_{j}^{0}\right)\left(\mathbf{U}_{\tilde{t} k 1}^{*} \mathbf{U}_{\tilde{t} l 1}+\mathbf{U}_{\tilde{t} k 2}^{*} \mathbf{U}_{\tilde{t} l 2}\right)\right], \\
c_{4}(h, h) & =c_{\alpha}^{2}, \\
c_{4}(H, H) & =s_{\alpha}^{2}, \\
c_{4}(A, A) & =c_{\beta}^{2}, \\
c_{4}(G, G) & =s_{\beta}^{2}, \\
c_{4}(h, H) & =c_{\alpha} s_{\alpha}, \\
c_{4}(A, G) & =c_{\beta} s_{\beta} .
\end{aligned}
$$

$$
\begin{aligned}
& =-i h_{t}^{2}\left[c_{4}^{\tilde{t}}\left(\Phi_{i}^{-}, \Phi_{j}^{+}\right) \mathbf{U}_{\tilde{t} k 2}^{*} \mathbf{U}_{\tilde{t} l 2}\right], \\
& c_{4}^{\tilde{t}}\left(H^{-}, H^{+}\right)=c_{\beta}^{2},
\end{aligned}
$$$$
c_{4}^{\tilde{t}}\left(G^{-}, G^{+}\right)=s_{\beta}^{2} \text {, }
$$$$
c_{4}^{\tilde{t}}\left(H^{-}, G^{+}\right)=c_{\beta} s_{\beta},
$$$$
c_{4}^{\tilde{t}}\left(G^{-}, H^{+}\right)=c_{\beta} s_{\beta} \text {. }
$$

$$
\begin{aligned}
c_{4}^{\tilde{b}}\left(H^{-}, H^{+}\right) & =c_{\beta}^{2}, \\
c_{4}^{\tilde{b}}\left(G^{-}, G^{+}\right) & =s_{\beta}^{2}, \\
c_{4}^{\tilde{b}}\left(H^{-}, G^{+}\right) & =c_{\beta} s_{\beta}, \\
c_{4}^{\tilde{b}}\left(G^{-}, H^{+}\right) & =c_{\beta} s_{\beta} .
\end{aligned}
$$

$$
\begin{aligned}
&=-i C\left(\Phi^{0}, \Phi^{-}, \tilde{t}_{k}, \tilde{b}_{1}\right)=-i C\left(\Phi^{0}, \Phi^{+}, \tilde{t}_{k}, \tilde{b}_{1}\right)^{*} \\
& c_{4}\left(h, H^{-}\right)=\frac{-c_{\alpha} c_{\beta}}{\sqrt{2}}, \quad c_{4}\left(H, H^{-}\right)=\frac{-s_{\alpha} c_{\beta}}{\sqrt{2}}, \\
& c_{4}\left(h, G^{-}\right)=\frac{-c_{\alpha} s_{\beta}}{\sqrt{2}}, \quad c_{4}\left(H, G^{-}\right)=\frac{-s_{\alpha} s_{\beta}}{\sqrt{2}}, \\
& c_{4}\left(A, H^{-}\right)=\frac{-i c_{\beta}^{2}}{\sqrt{2}}, \quad c_{4}\left(G, H^{-}\right)=\frac{-i c_{\beta} s_{\beta}}{\sqrt{2}}, \\
& c_{4}\left(A, G^{-}\right)=\frac{-i c_{\beta} s_{\beta}}{\sqrt{2}}, \quad c_{4}\left(G, G^{-}\right)=\frac{-i s_{\beta}^{2}}{\sqrt{2}} .
\end{aligned}
$$



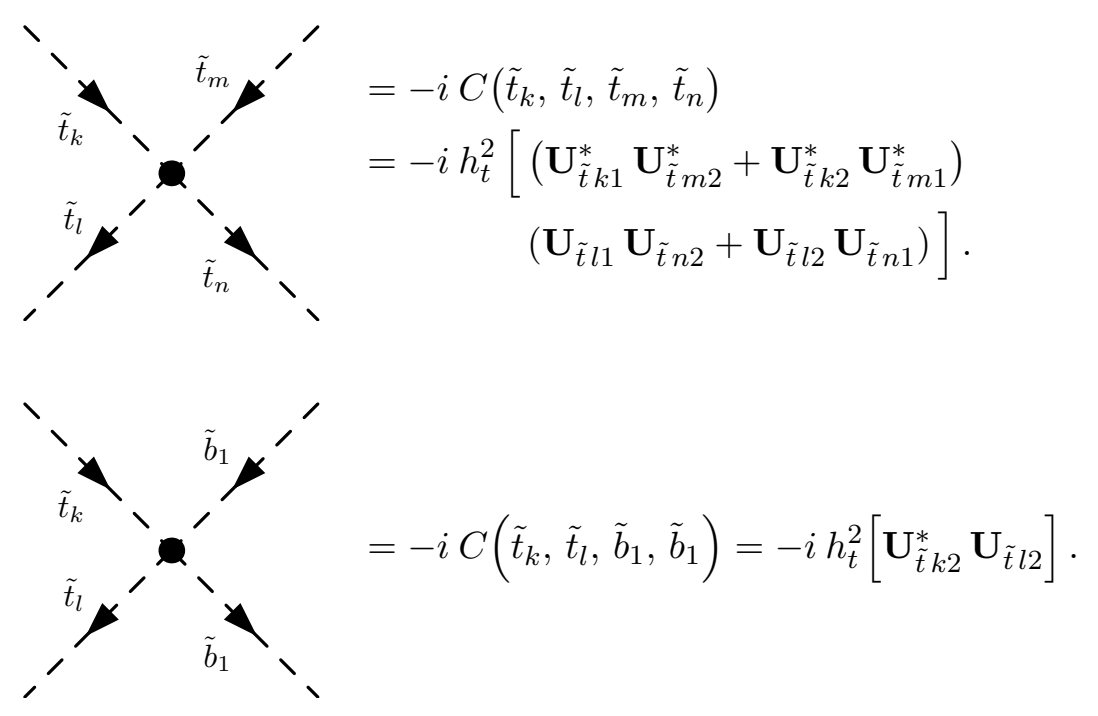

$$
\begin{aligned}
=-i C\left(\Phi^{0}, t, t\right)= & -i h_{t}\left[c_{3}\left(\Phi^{0}\right)\right]\left(\begin{array}{c}
1 \\
\operatorname{sign}\left(\Phi^{0}\right)
\end{array}\right) \\
\left\{c_{3}(h), \operatorname{sign}(h)\right\} & =\left\{\frac{c_{\alpha}}{\sqrt{2}}, 1\right\}, \\
\left\{c_{3}(H), \operatorname{sign}(H)\right\} & =\left\{\frac{s_{\alpha}}{\sqrt{2}}, 1\right\}, \\
\left\{c_{3}(A), \operatorname{sign}(A)\right\} & =\left\{\frac{i c_{\beta}}{\sqrt{2}},-1\right\}, \\
\left\{c_{3}(G), \operatorname{sign}(G)\right\} & =\left\{\frac{i s_{\beta}}{\sqrt{2}},-1\right\} .
\end{aligned}
$$$$
\begin{aligned}
& =-i C\left(\Phi^{-}, t, \bar{b}\right)=-i h_{t}\left[c_{3}\left(\Phi^{-}\right)\right]\left(\begin{array}{l}
0 \\
1
\end{array}\right), \\
& c_{3}\left(H^{-}\right)=-c_{\beta}, \\
& c_{3}\left(G^{-}\right)=-s_{\beta} .
\end{aligned}
$$

$$
\begin{aligned}
& =-i C\left(\Phi^{+}, \bar{t}, b\right)=-i h_{t}\left[\bar{c}_{3}\left(\Phi^{+}\right)\right]\left(\begin{array}{l}
1 \\
0
\end{array}\right), \\
& \bar{c}_{3}\left(H^{+}\right)=-c_{\beta}, \\
& \bar{c}_{3}\left(G^{+}\right)=-s_{\beta} .
\end{aligned}
$$




$$
\begin{aligned}
&=-i C\left(\Phi^{0}, \tilde{t}_{k}, \tilde{t}_{l}\right) \\
&=-i h_{t}\left[\begin{array}{c}
c_{m}\left(\Phi^{0}\right)\left(\mathbf{U}_{\tilde{t} k 1}^{*} \mathbf{U}_{\tilde{t} l 1}+\mathbf{U}_{\tilde{t} k 2}^{*} \mathbf{U}_{\tilde{t} l 2}\right) m_{t} \\
+c_{\mu}\left(\Phi^{0}\right)\left(\operatorname{sign}\left(\Phi^{0}\right) \mathbf{U}_{\tilde{t} k 2}^{*} \mathbf{U}_{\tilde{t} l l} \mu+\mathbf{U}_{\tilde{t} k 1}^{*} \mathbf{U}_{\tilde{t} l 2} \mu^{*}\right) \\
\left.+c_{A}\left(\Phi^{0}\right)\left(\operatorname{sign}\left(\Phi^{0}\right) \mathbf{U}_{\tilde{t} k 2}^{*} \mathbf{U}_{\tilde{t} l 1} A_{t}^{*}+\mathbf{U}_{\tilde{t} k 1}^{*} \mathbf{U}_{\tilde{t} l 2} A_{t}\right)\right]
\end{array}\right. \\
&\left\{c_{m}(h), c_{\mu}(h), c_{A}(h), \operatorname{sign}(h)\right\}=\left\{\sqrt{2} c_{\alpha}, \frac{s_{\alpha}}{\sqrt{2}}, \frac{c_{\alpha}}{\sqrt{2}}, 1\right\}, \\
&\left\{c_{m}(H), c_{\mu}(H), c_{A}(H), \operatorname{sign}(H)\right\}=\left\{\sqrt{2} s_{\alpha}, \frac{-c_{\alpha}}{\sqrt{2}}, \frac{s_{\alpha}}{\sqrt{2}}, 1\right\} \\
&\left\{c_{m}(A), c_{\mu}(A), c_{A}(A), \operatorname{sign}(A)\right\}=\left\{0, \frac{i s_{\beta}}{\sqrt{2}}, \frac{i c_{\beta}}{\sqrt{2}},-1\right\}, \\
&\left\{c_{m}(G), c_{\mu}(G), c_{A}(G), \operatorname{sign}(G)\right\}=\left\{0, \frac{-i c_{\beta}}{\sqrt{2}}, \frac{i s_{\beta}}{\sqrt{2}},-1\right\}
\end{aligned}
$$
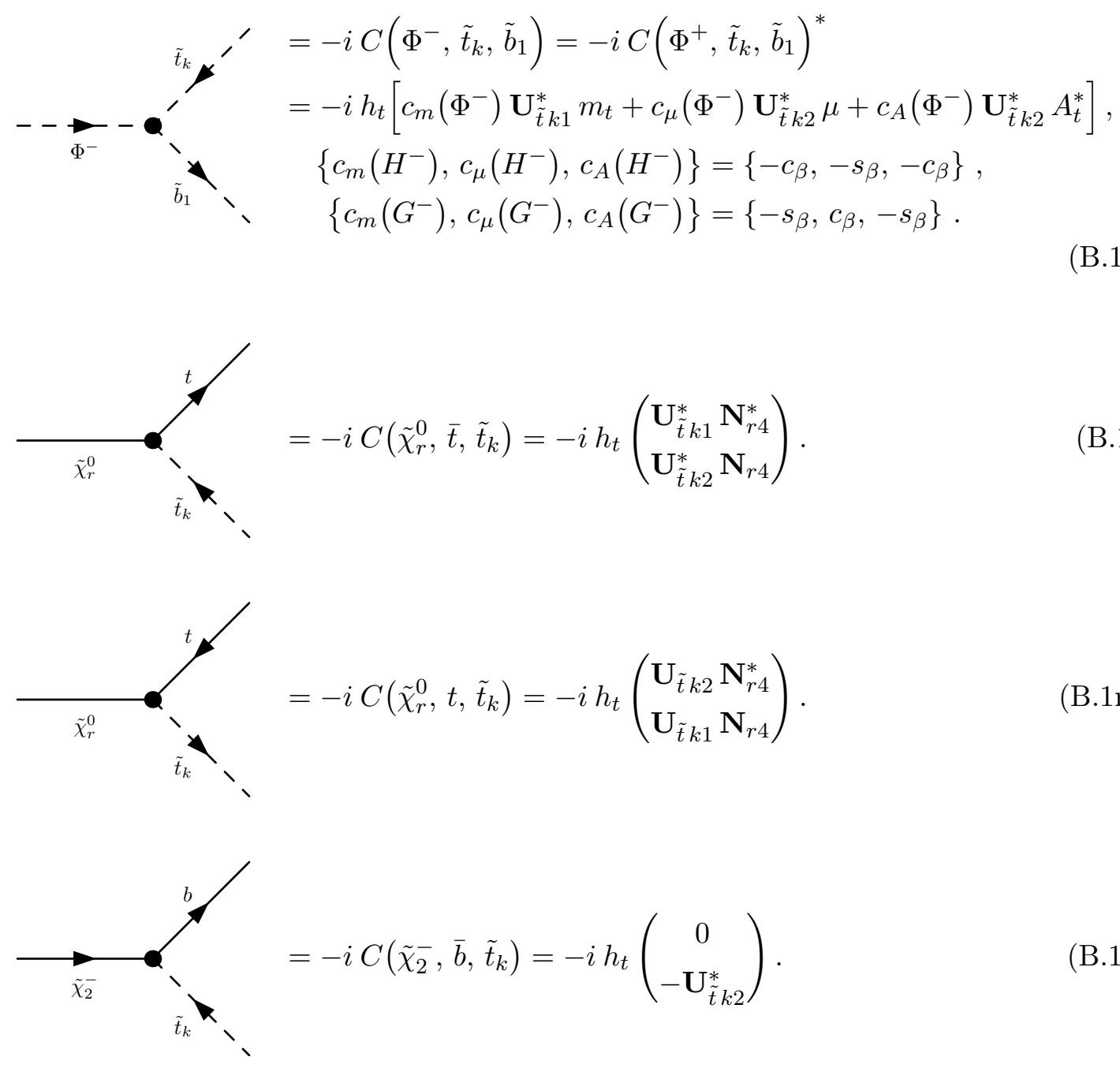


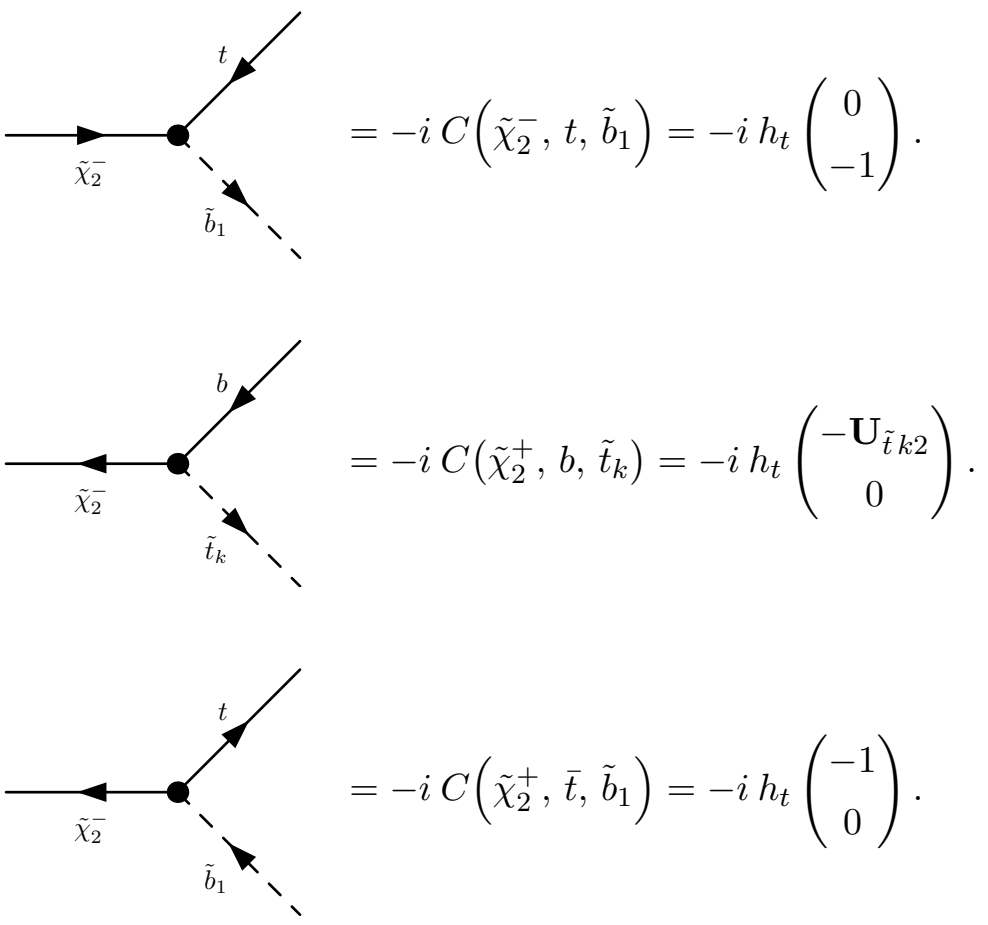

\section{B.2 Counterterm vertices}

The following one-loop counterterms for the two, three- and four-point vertices appear as insertions in the two-loop diagrams with subrenormalization for masses and couplings. For the two-point vertices we have

$$
\begin{aligned}
\vec{t} \times \vec{t} & =i \delta^{(1)} m_{t} . \\
--{\overrightarrow{\tilde{t}_{i}}}^{-}-\boldsymbol{*}-{\overrightarrow{\tilde{t}_{j}}}^{--} & =i \delta^{(1)} m_{\tilde{t}_{i} \tilde{t}_{j}}^{2} . \\
--{\overrightarrow{\tilde{b}_{1}}}^{-}-\boldsymbol{*}-{\overrightarrow{\tilde{b}_{1}}}^{--} & =i \delta^{(1)} m_{\tilde{b}_{1}}^{2} \equiv i \delta^{(1)} m_{\tilde{q}_{3}}^{2} .
\end{aligned}
$$

To shorten the notation for the three- and four-point vertices, the previously defined tree-level couplings $C(\ldots)$ are re-utilized; their corresponding one-loop counterterms are named $\delta^{(1)} C(\ldots)$. Since each of the vertices contains the top-Yukawa coupling $h_{t}$, its renormalization constant $\delta^{(1)} h_{t}$, given by

$$
\delta^{(1)} h_{t}=h_{t}\left(\frac{\delta^{(1)} m_{t}}{m_{t}}-\frac{\delta^{(1)} M_{W}}{M_{W}}-\frac{\delta^{(1)} s_{\mathrm{w}}}{s_{\mathrm{w}}}-\frac{\delta^{(1)} s_{\beta}}{s_{\beta}}\right),
$$

is part of each vertex counterterm [the renormalization constant $\delta^{(1)} e / e$ is omitted since there are no contributions of $\left.\mathcal{O}\left(\alpha_{t}\right)\right]$. Also the field-renormalization constants of the Higgs bosons are kept; all other field-renormalization constants cancel out in the sum of the full set of Feynman diagrams, since the corresponding particles exclusively appear in internal propagators. 


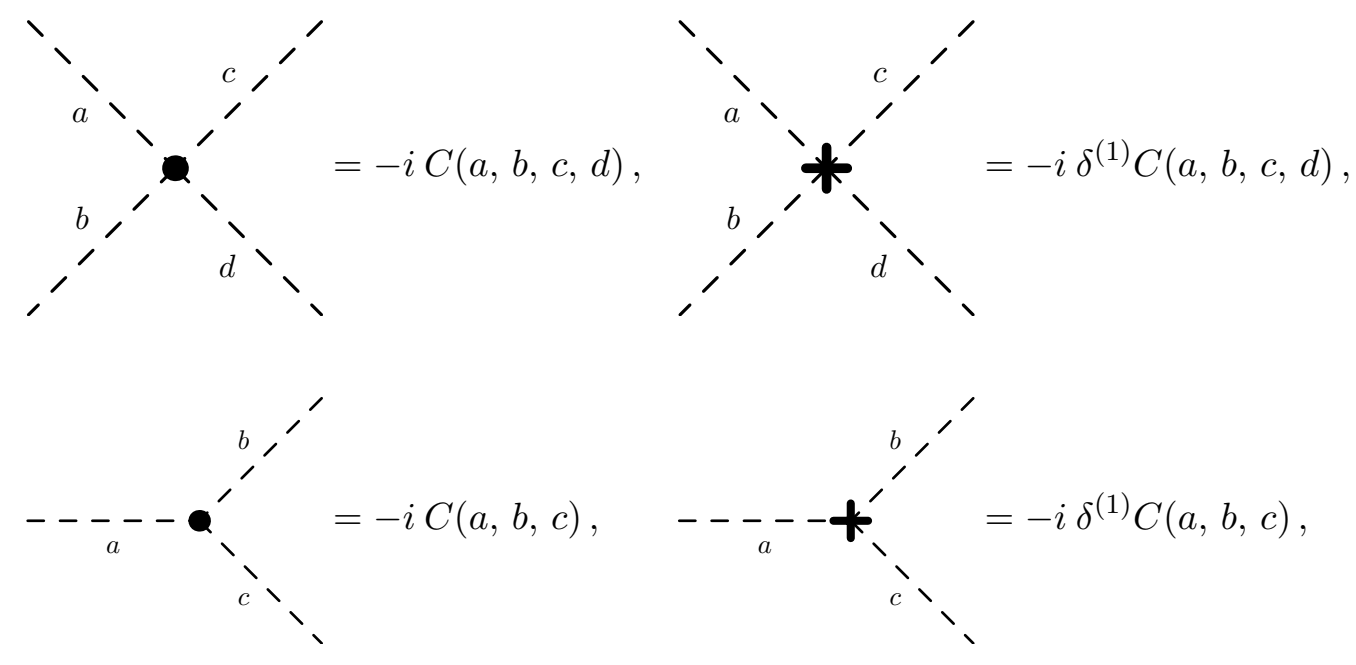

$$
\begin{aligned}
\delta^{(1)} C\left(\Phi_{i}^{0}, \Phi_{j}^{0}, \tilde{t}_{k}, \tilde{t}_{l}\right) & =C\left(\Phi_{i}^{0}, \Phi_{j}^{0}, \tilde{t}_{k}, \tilde{t}_{l}\right)\left(\frac{2 \delta^{(1)} h_{t}}{h_{t}}+\delta^{(1)} Z_{\mathcal{H}_{2}}\right) \\
\delta^{(1)} C\left(\Phi_{i}^{-}, \Phi_{j}^{+}, \tilde{t}_{k}, \tilde{t}_{l}\right) & =C\left(\Phi_{i}^{-}, \Phi_{j}^{+}, \tilde{t}_{k}, \tilde{t}_{l}\right)\left(\frac{2 \delta^{(1)} h_{t}}{h_{t}}+\delta^{(1)} Z_{\mathcal{H}_{2}}\right) \\
\delta^{(1)} C\left(\Phi_{i}^{-}, \Phi_{j}^{+}, \tilde{b}_{1}, \tilde{b}_{1}\right) & =C\left(\Phi_{i}^{-}, \Phi_{j}^{+}, \tilde{b}_{1}, \tilde{b}_{1}\right)\left(\frac{2 \delta^{(1)} h_{t}}{h_{t}}+\delta^{(1)} Z_{\mathcal{H}_{2}}\right) \\
\delta^{(1)} C\left(\Phi^{0}, \Phi^{-}, \tilde{t}_{k}, \tilde{b}_{1}\right) & =C\left(\Phi^{0}, \Phi^{-}, \tilde{t}_{k}, \tilde{b}_{1}\right)\left(\frac{2 \delta^{(1)} h_{t}}{h_{t}}+\delta^{(1)} Z_{\mathcal{H}_{2}}\right) \\
\delta^{(1)} C\left(\tilde{t}_{k}, \tilde{t}_{l}, \tilde{t}_{m}, \tilde{t}_{n}\right) & =C\left(\tilde{t}_{k}, \tilde{t}_{l}, \tilde{t}_{m}, \tilde{t}_{n}\right)\left(\frac{2 \delta^{(1)} h_{t}}{h_{t}}\right) \\
\delta^{(1)} C\left(\tilde{t}_{k}, \tilde{t}_{l}, \tilde{b}_{1}, \tilde{b}_{1}\right) & =C\left(\tilde{t}_{k}, \tilde{t}_{l}, \tilde{b}_{1}, \tilde{b}_{1}\right)\left(\frac{2 \delta^{(1)} h_{t}}{h_{t}}\right) \\
\delta^{(1)} C\left(\Phi^{0}, t, t\right) & =C\left(\Phi^{0}, t, t\right)\left(\frac{\delta^{(1)} h_{t}}{h_{t}}+\frac{1}{2} \delta^{(1)} Z_{\mathcal{H}_{2}}\right) \\
\delta^{(1)} C\left(\Phi^{-}, t, \bar{b}\right) & =C\left(\Phi^{-}, t, \bar{b}\right)\left(\frac{\delta^{(1)} h_{t}}{h_{t}}+\frac{1}{2} \delta^{(1)} Z_{\mathcal{H}_{2}}\right) \\
\delta^{(1)} C\left(\Phi^{+}, \bar{t}, b\right) & =C\left(\Phi^{+}, \bar{t}, b\right)\left(\frac{\delta^{(1)} h_{t}}{h_{t}}+\frac{1}{2} \delta^{(1)} Z_{\mathcal{H}_{2}}\right)
\end{aligned}
$$




$$
\begin{aligned}
& \delta^{(1)} C\left(\Phi^{0}, \tilde{t}_{k}, \tilde{t}_{l}\right)=C\left(\Phi^{0}, \tilde{t}_{k}, \tilde{t}_{l}\right)\left(\frac{\delta^{(1)} h_{t}}{h_{t}}\right) \\
& +h_{t}\left\{c_{m}\left(\Phi^{\emptyset}\right)\left(\mathbf{U}_{\tilde{t} k 1}^{*} \mathbf{U}_{\tilde{t} l 1}+\mathbf{U}_{\tilde{t} k 2}^{*} \mathbf{U}_{\tilde{t} l 2}\right) m_{t}\left(\frac{\delta^{(1)} m_{t}}{m_{t}}+\frac{1}{2} \delta^{(1)} Z_{\mathcal{H}_{2}}\right)\right. \\
& +c_{A}\left(\Phi^{0}\right)\left[\operatorname{sign}\left(\Phi^{0}\right) \mathbf{U}_{\tilde{t} k 2}^{*} \mathbf{U}_{\tilde{t} l 1} A_{t}^{*}\left(\frac{\delta^{(1)} A_{t}^{*}}{A_{t}^{*}}+\frac{1}{2} \delta^{(1)} Z_{\mathcal{H}_{2}}\right)\right. \\
& \left.+\mathbf{U}_{\tilde{t} k 1}^{*} \mathbf{U}_{\tilde{t} l 2} A_{t}\left(\frac{\delta^{(1)} A_{t}}{A_{t}}+\frac{1}{2} \delta^{(1)} Z_{\mathcal{H}_{2}}\right)\right] \\
& +c_{\mu}\left(\Phi^{0}\right)\left[\operatorname{sign}\left(\Phi^{0}\right) \mathbf{U}_{\tilde{t} k 2}^{*} \mathbf{U}_{\tilde{t} l 1} \mu\left(\frac{\delta^{(1)} \mu}{\mu}+\frac{1}{2} \delta^{(1)} Z_{\mathcal{H}_{1}}\right)\right\}, \\
& \left.+\mathbf{U}_{\tilde{t} k 1}^{*} \mathbf{U}_{\tilde{t} l 2} \mu^{*}\left(\frac{\delta^{(1)} \mu^{*}}{\mu^{*}}+\frac{1}{2} \delta^{(1)} Z_{\mathcal{H}_{1}}\right)\right]
\end{aligned}
$$$$
\delta^{(1)} C\left(\Phi^{-}, \tilde{t}_{k}, \tilde{b}_{1}\right)=C\left(\Phi^{-}, \tilde{t}_{k}, \tilde{b}_{1}\right)\left(\frac{\delta^{(1)} h_{t}}{h_{t}}\right)
$$$$
+h_{t}\left[c_{\mu}\left(\Phi^{-}\right) \mathbf{U}_{\tilde{t} k 2}^{*} \mu\left(\frac{\delta^{(1)} \mu}{\mu}+\frac{1}{2} \delta^{(1)} Z_{\mathcal{H}_{1}}\right)\right.
$$$$
+c_{A}\left(\Phi^{-}\right) \mathbf{U}_{\tilde{t} k 2}^{*} A_{t}^{*}\left(\frac{\delta^{(1)} A_{t}^{*}}{A_{t}^{*}}+\frac{1}{2} \delta^{(1)} Z_{\mathcal{H}_{2}}\right)
$$$$
\left.+c_{m}\left(\Phi^{-}\right) \mathbf{U}_{\tilde{t} k 1}^{*} m_{t}\left(\frac{\delta^{(1)} m_{t}}{m_{t}}+\frac{1}{2} \delta^{(1)} Z_{\mathcal{H}_{2}}\right)\right] \text {, }
$$$$
\delta^{(1)} C\left(\tilde{\chi}_{r}^{0}, \bar{t}, \tilde{t}_{k}\right)=C\left(\tilde{\chi}_{r}^{0}, \bar{t}, \tilde{t}_{k}\right)\left(\frac{\delta^{(1)} h_{t}}{h_{t}}\right)
$$$$
{ }^{(1)} C\left(\tilde{\chi}_{r}^{0}, t, \tilde{t}_{k}\right)=C\left(\tilde{\chi}_{r}^{0}, t, \tilde{t}_{k}\right)\left(\frac{\delta^{(1)} h_{t}}{h_{t}}\right),
$$$$
\delta^{(1)} C\left(\tilde{\chi}_{2}^{-}, \bar{b}, \tilde{t}_{k}\right)=C\left(\tilde{\chi}_{2}^{-}, \bar{b}, \tilde{t}_{k}\right)\left(\frac{\delta^{(1)} h_{t}}{h_{t}}\right),
$$$$
\delta^{(1)} C\left(\tilde{\chi}_{2}^{-}, t, \tilde{t}_{k}\right)=C\left(\tilde{\chi}_{2}^{-}, t, \tilde{t}_{k}\right)\left(\frac{\delta^{(1)} h_{t}}{h_{t}}\right),
$$$$
\delta^{(1)} C\left(\tilde{\chi}_{2}^{+}, b, \tilde{t}_{k}\right)=C\left(\tilde{\chi}_{2}^{+}, b, \tilde{t}_{k}\right)\left(\frac{\delta^{(1)} h_{t}}{h_{t}}\right),
$$$$
\delta^{(1)} C\left(\tilde{\chi}_{2}^{+}, \bar{t}, \tilde{b}_{1}\right)=C\left(\tilde{\chi}_{2}^{+}, \bar{t}, \tilde{b}_{1}\right)\left(\frac{\delta^{(1)} h_{t}}{h_{t}}\right) .
$$

The counterterms of eq. (B.5j) and eq. (B.5k) should be emphasized, because they are the only ones which cannot be simply expressed as a product of a tree-level coupling and the counterterm of the top-Yukawa coupling and field renormalization. 


\section{Loop integrals}

The analytical evaluation of the $\mathcal{O}\left(\alpha_{t}^{2}\right)$ contributions requires the following explicit expressions for one-loop and two-loop integrals.

\section{C.1 One-loop functions}

In the following all required one-loop integrals are listed up to $\mathcal{O}\left(\epsilon^{1}\right)$, where $\epsilon=(4-D) / 2$ parametrizes the divergent parts. $D$ is the dimension of the integrated momentum and $\mu_{\mathrm{D}}$ depicts the regularization parameter, so that

$$
\int \mathrm{d}^{4} q \rightarrow \mu_{\mathrm{D}}^{4-D} \int \mathrm{d}^{D} q
$$

The reduction to scalar integrals as described first by ref. [78] has been used. The scalar integrals have been re-evaluated by using the technique of Feynman parameters.

$$
\begin{aligned}
A_{0}(0)= & 0 \\
A_{0}\left(m^{2}\right)= & \frac{m^{2}}{\epsilon}-m^{2}\left\{\mathrm{~L}\left(m^{2}\right)\right\}+m^{2} \epsilon\left\{\frac{1}{2}+\frac{\pi^{2}}{12}+\frac{1}{2}\left[\mathrm{~L}\left(m^{2}\right)\right]^{2}\right\}, \\
B_{0}\left(p^{2}, 0,0\right)= & \frac{1}{\epsilon}+\left\{1+C+\log \left(-\frac{\mu_{\mathrm{D}}}{p^{2}-i \epsilon^{\prime}}\right)\right\} \\
& +\epsilon\left\{2-\frac{\pi^{2}}{12}+\frac{1}{2}\left[1+C+\log \left(-\frac{\mu_{\mathrm{D}}}{p^{2}-i \epsilon^{\prime}}\right)\right]^{2}\right\}, \\
B_{0}\left(0,0, m^{2}\right)= & B_{0}\left(0, m^{2}, 0\right)=\frac{A_{0}\left(m^{2}\right)}{m^{2}}, \\
B_{0}\left(0, m^{2}, m^{2}\right)= & (1-\epsilon) \frac{A_{0}\left(m^{2}\right)}{m^{2}}, \\
B_{0}\left(0, m_{1}^{2}, m_{2}^{2}\right)= & \frac{A_{0}\left(m_{1}^{2}\right)-A_{0}\left(m_{2}^{2}\right)}{m_{1}^{2}-m_{2}^{2}}, \\
B_{0}\left(m^{2}, 0, m^{2}\right)= & \frac{1}{\epsilon}+\left\{1-\mathrm{L}\left(m^{2}\right)\right\}+\epsilon\left\{2+\frac{\pi^{2}}{12}+\frac{1}{2}\left[1-\mathrm{L}\left(m^{2}\right)\right]^{2}\right\}, \\
B_{0}\left(m^{2}, m^{2}, 0\right)= & B_{0}\left(m^{2}, 0, m^{2}\right), \\
B_{0}\left(m_{1}^{2}, 0, m_{2}^{2}\right)= & \frac{1}{\epsilon}+\left\{\frac{m_{2}^{2}}{m_{1}^{2}}\left[1-\mathrm{L}\left(m_{2}^{2}\right)\right]+\frac{m_{1}^{2}-m_{2}^{2}}{m_{1}^{2}}\left[1+C+\log \left(\frac{\mu_{\mathrm{D}}}{m_{2}^{2}-m_{1}^{2}}\right)\right]\right\} \\
& +\epsilon\left\{\frac{m_{1}^{2}-m_{2}^{2}}{2 m_{1}^{2}}\left[\left(1+C+\log \left(\frac{\mu_{\mathrm{D}}}{m_{2}^{2}-m_{1}^{2}}\right)\right)^{2}-2 \mathrm{Li}_{2}\left(\frac{-m_{1}^{2}}{m_{2}^{2}-m_{1}^{2}}\right)\right]\right. \\
& +\epsilon\left\{2+\frac{\pi^{2}}{12}+\frac{m_{2}^{2}}{2 m_{1}^{2}}\left[1-\mathrm{L}\left(m_{2}^{2}\right)\right]^{2}\right\}, \\
& \frac{1}{\epsilon}+\left\{\frac{m_{1}\left[1-\mathrm{L}\left(m_{1}^{2}\right)\right] \pm m_{2}\left[1-\mathrm{L}\left(m_{2}^{2}\right)\right]}{m_{1} \pm m_{2}}\right\} \\
B_{0}\left(m_{1}^{2}, m_{2}^{2}, 0\right)= & B_{0}\left(m_{1}^{2}, 0, m_{2}^{2}\right), \\
B_{0}\left(\left(m_{1} \pm m_{2}\right)^{2},\right. & \left.\left.m_{1}^{2}, m_{2}^{2}\right)=\mathrm{L}\left(m_{1}^{2}\right)\right]^{2} \pm m_{2}\left[1-\mathrm{L}\left(m_{2}^{2}\right)\right]^{2} \\
& \left.+m_{2}\right) \\
&
\end{aligned}
$$




$$
\begin{aligned}
B_{0}\left(p^{2}, m_{1}^{2}, m_{2}^{2}\right)=\frac{1}{\epsilon}+\left\{\frac{m_{1}^{2}-m_{2}^{2}+p^{2}}{2 p^{2}}\left[1-\mathrm{L}\left(m_{1}^{2}\right)\right]+\frac{m_{2}^{2}-m_{1}^{2}+p^{2}}{2 p^{2}}\left[1-\mathrm{L}\left(m_{2}^{2}\right)\right]\right. \\
\left.+\frac{R}{2 p^{2}}\left[\log \left(m_{1}^{2}+m_{2}^{2}-p^{2}+R\right)+\log \left(\frac{1}{m_{1}^{2}+m_{2}^{2}-p^{2}-R}\right)\right]\right\} \\
+\epsilon\left\{2+\frac{\pi^{2}}{12}+\frac{m_{1}^{2}-m_{2}^{2}+p^{2}}{4 p^{2}}\left[1-\mathrm{L}\left(m_{1}^{2}\right)\right]^{2}+\frac{m_{2}^{2}-m_{1}^{2}+p^{2}}{4 p^{2}}\left[1-\mathrm{L}\left(m_{2}^{2}\right)\right]^{2}\right. \\
-\frac{R}{4 p^{2}}\left[\left(1-\mathrm{L}\left(m_{1}^{2}\right)\right)\left(\log \left(m_{1}^{2}-m_{2}^{2}+p^{2}+R\right)-\log \left(m_{2}^{2}-m_{1}^{2}-p^{2}+R\right)\right)\right. \\
+\left(1-\mathrm{L}\left(m_{2}^{2}\right)\right)\left(\log \left(m_{2}^{2}-m_{1}^{2}+p^{2}+R\right)-\log \left(m_{1}^{2}-m_{2}^{2}-p^{2}+R\right)\right) \\
+\left(-1-\mathrm{L}\left(-p^{2}\right)+2 \mathrm{~L}(R)\right) \\
\quad\left(\log \left(m_{1}^{2}+m_{2}^{2}-p^{2}+R\right)+\log \left(\frac{1}{m_{1}^{2}+m_{2}^{2}-p^{2}-R}\right)\right) \\
+2\left(\operatorname{Li}_{2}\left(\frac{m_{1}^{2}-m_{2}^{2}-p^{2}+R}{2 R}\right)-\operatorname{Li}_{2}\left(\frac{m_{2}^{2}-m_{1}^{2}+p^{2}+R}{2 R}\right)\right) \\
\left.\left.+2\left(\operatorname{Li}_{2}\left(\frac{m_{2}^{2}-m_{1}^{2}-p^{2}+R}{2 R}\right)-\operatorname{Li}_{2}\left(\frac{m_{1}^{2}-m_{2}^{2}+p^{2}+R}{2 R}\right)\right)\right]\right\},
\end{aligned}
$$

$$
B_{1}\left(0, m_{1}^{2}, m_{2}^{2}\right)=-\frac{1}{2} B_{0}\left(0, m_{1}^{2}, m_{2}^{2}\right)+\frac{m_{2}^{2}-m_{1}^{2}}{2} B_{0}^{\prime}\left(0, m_{1}^{2}, m_{2}^{2}\right),
$$

$$
B_{1}\left(p^{2}, m_{1}^{2}, m_{2}^{2}\right)=\frac{1}{2 p^{2}}\left[A_{0}\left(m_{1}^{2}\right)-A_{0}\left(m_{2}^{2}\right)-\left(p^{2}-m_{2}^{2}+m_{1}^{2}\right) B_{0}\left(p^{2}, m_{1}^{2}, m_{2}^{2}\right)\right],
$$

$B_{00}\left(p^{2}, m_{1}^{2}, m_{2}^{2}\right)=\frac{1}{2(3-2 \epsilon)}\left[A_{0}\left(m_{2}^{2}\right)+2 m_{1}^{2} B_{0}\left(p^{2}, m_{1}^{2}, m_{2}^{2}\right)\right.$

$$
\left.+\left(p^{2}-m_{2}^{2}+m_{1}^{2}\right) B_{1}\left(p^{2}, m_{1}^{2}, m_{2}^{2}\right)\right],
$$

$$
\begin{aligned}
B_{0}^{\prime}(0,0,0)= & 0 \\
B_{0}^{\prime}\left(0,0, m^{2}\right)= & \frac{1}{2 m^{2}}+\frac{\epsilon}{2 m^{2}}\left\{\frac{1}{2}-\mathrm{L}\left(m^{2}\right)\right\}, \\
B_{0}^{\prime}\left(0, m^{2}, 0\right)= & B_{0}^{\prime}\left(0,0, m^{2}\right), \\
B_{0}^{\prime}\left(0, m^{2}, m^{2}\right)= & \frac{1}{6 m^{2}}+\frac{\epsilon}{6 m^{2}}\left\{-1-\mathrm{L}\left(m^{2}\right)\right\}, \\
B_{0}^{\prime}\left(0, m_{1}^{2}, m_{2}^{2}\right)= & \frac{1}{2\left(m_{1}^{2}-m_{2}^{2}\right)^{3}}\left\{m_{1}^{4}-m_{2}^{4}+2 m_{1}^{2} m_{2}^{2} \log \left(\frac{m_{2}^{2}}{m_{1}^{2}}\right)\right\} \\
& +\frac{\epsilon}{2\left(m_{1}^{2}-m_{2}^{2}\right)^{3}}\left\{m_{1}^{4}\left[\frac{1}{2}-\mathrm{L}\left(m_{1}^{2}\right)\right]-m_{2}^{4}\left[\frac{1}{2}-\mathrm{L}\left(m_{2}^{2}\right)\right]\right. \\
& \left.\quad+m_{1}^{2} m_{2}^{2}\left[\left(\mathrm{~L}\left(m_{1}^{2}\right)\right)^{2}-\left(\mathrm{L}\left(m_{2}^{2}\right)\right)^{2}\right]\right\},
\end{aligned}
$$

$C_{0}\left(0,0,0, m^{2}, m^{2}, m^{2}\right)=-\frac{\epsilon}{2 m^{2}} B_{0}\left(0, m^{2}, m^{2}\right)$,

$$
\begin{aligned}
\mathrm{L}\left(m^{2}\right) & =\log \left(\frac{m^{2}}{\mu_{\mathrm{D}}}\right)-C, \\
C & =1-\gamma_{\mathrm{E}}+\log (4 \pi), \\
R & =\sqrt{m_{1}^{4}+m_{2}^{4}+p^{4}-2 m_{1}^{2} m_{2}^{2}-2 m_{2}^{2} p^{2}-2 p^{2} m_{1}^{2}} .
\end{aligned}
$$




\section{C.2 Two-loop functions}

The notation of the two-loop integrals follows the conventions which have been introduced by refs. $[58,79]$. After reducing the appearing two-loop integrals to a set of master integrals and applying the approximation of a vanishing external momentum, only the following function is left which cannot be completely expressed in terms of one-loop functions. The result is taken from ref. [80] and reordered in the given way. Up to $\mathcal{O}\left(\epsilon^{0}\right)$ it reads:

$$
\begin{aligned}
\mathrm{T}_{134}\left(m_{1}^{2}, m_{2}^{2}, m_{3}^{2}\right)= & \frac{1-\epsilon}{2(1-2 \epsilon)}\left\{\frac{\left[A_{0}\left(m_{1}^{2}\right)\right]^{2}}{m_{1}^{2}}+\frac{\left[A_{0}\left(m_{2}^{2}\right)\right]^{2}}{m_{2}^{2}}+\frac{\left[A_{0}\left(m_{3}^{2}\right)\right]^{2}}{m_{3}^{2}}\right\} \\
& +\Phi^{\mathrm{cyc}}\left(m_{1}^{2}, m_{2}^{2}, m_{3}^{2}\right) \\
\Phi^{\mathrm{cyc}}\left(m^{2}, 0,0\right)= & m^{2} \frac{\pi^{2}}{6}, \\
\Phi^{\mathrm{cyc}}\left(m_{1}^{2}, m_{2}^{2}, 0\right)= & m_{1}^{2} \operatorname{Li}_{2}\left(\frac{m_{1}^{2}-m_{2}^{2}}{m_{1}^{2}}\right)+m_{2}^{2} \operatorname{Li}_{2}\left(\frac{m_{2}^{2}-m_{1}^{2}}{m_{2}^{2}}\right), \\
\Phi^{\mathrm{cyc}}\left(m_{1}^{2}, m_{2}^{2}, m_{3}^{2}\right)= & -\frac{m_{1}^{2}}{2} \log \left(\frac{m_{1}^{2}}{m_{2}^{2}}\right) \log \left(\frac{m_{1}^{2}}{m_{3}^{2}}\right)-\frac{m_{2}^{2}}{2} \log \left(\frac{m_{2}^{2}}{m_{3}^{2}}\right) \log \left(\frac{m_{2}^{2}}{m_{1}^{2}}\right)-\frac{m_{3}^{2}}{2} \log \left(\frac{m_{3}^{2}}{m_{1}^{2}}\right) \log \left(\frac{m_{3}^{2}}{m_{2}^{2}}\right) \\
& +R\left[\frac{\pi^{2}}{6}-\frac{1}{2} \log \left(\frac{m_{1}^{2}}{m_{3}^{2}}\right) \log \left(\frac{m_{2}^{2}}{m_{3}^{2}}\right)+\log \left(\frac{m_{1}^{2}-m_{2}^{2}+m_{3}^{2}-R}{2 m_{3}^{2}}\right) \log \left(\frac{m_{2}^{2}-m_{1}^{2}+m_{3}^{2}-R}{2 m_{3}^{2}}\right)\right. \\
& \left.-\operatorname{Li}\left(\frac{m_{1}^{2}-m_{2}^{2}+m_{3}^{2}-R}{2 m_{3}^{2}}\right)-\operatorname{Li}_{2}\left(\frac{m_{2}^{2}-m_{1}^{2}+m_{3}^{2}-R}{2 m_{3}^{2}}\right)\right], \\
R= & \sqrt{m_{1}^{4}+m_{2}^{4}+m_{3}^{4}-2 m_{1}^{2} m_{2}^{2}-2 m_{2}^{2} m_{3}^{2}-2 m_{3}^{2} m_{1}^{2}} .
\end{aligned}
$$

The function $\Phi^{\text {cyc }}$ is cyclic in its arguments and contains only finite parts.

During the reduction to master integrals some terms can be expressed as products of one-loop integrals:

$$
\begin{aligned}
\mathrm{T}_{a b}\left(m_{1}^{2}, m_{2}^{2}\right)= & A_{0}\left(m_{1}^{2}\right) A_{0}\left(m_{2}^{2}\right), \\
\mathrm{T}_{a^{x} b y}(\underbrace{m_{1}^{2}, \ldots, m_{1}^{2}}_{x}, \underbrace{m_{2}^{2}, \ldots, m_{2}^{2}}_{y})= & \frac{x-3+\epsilon}{(1-x) m_{1}^{2}} \mathrm{~T}_{a^{x-1} b^{y}}(\underbrace{m_{1}^{2}, \ldots, m_{1}^{2}}_{x-1}, \underbrace{m_{2}^{2}, \ldots, m_{2}^{2}}_{y}) \\
& \text { for } x>1, y \geq 1, \\
\mathrm{~T}_{a^{x} b y}(\underbrace{m_{1}^{2}, \ldots, m_{1}^{2}}_{x}, \underbrace{m_{2}^{2}, \ldots, m_{2}^{2}}_{y})= & \frac{y-3+\epsilon}{(1-y) m_{2}^{2}} \mathrm{~T}_{a^{x} b^{y-1}}(\underbrace{m_{1}^{2}, \ldots, m_{1}^{2}}_{x}, \underbrace{m_{2}^{2}, \ldots, m_{2}^{2}}_{y-1}) \\
& \text { for } x \geq 1, y>1,
\end{aligned}
$$

with $a \neq b$ and $a, b \in\{1,3,4\}$.

All other appearing integrals can be reduced to eq. (C.8a) or eq. (C.9) by using the following formulas:

$$
\mathrm{T}_{11334}\left(m_{1}^{2}, m_{1}^{2}, m_{1}^{2}, m_{1}^{2}, 0\right)=\frac{2}{m_{1}^{2}} \mathrm{~T}_{1113}\left(m_{1}^{2}, m_{1}^{2}, m_{1}^{2}, m_{1}^{2}\right)-2 \mathrm{~T}_{11134}\left(m_{1}^{2}, m_{1}^{2}, m_{1}^{2}, m_{1}^{2}, 0\right)
$$




$$
\begin{aligned}
\mathrm{T}_{11334}\left(m_{1}^{2}, m_{1}^{2}, m_{2}^{2}, m_{2}^{2}, m_{3}^{2}\right)= & -\frac{\left(m_{1}^{2}-m_{2}^{2}\right)^{2}-m_{3}^{4}}{\left(m_{1}^{2}-m_{2}^{2}\right)^{2}+m_{3}^{4}}\left[\mathrm{~T}_{11134}\left(m_{1}^{2}, m_{1}^{2}, m_{1}^{2}, m_{2}^{2}, m_{3}^{2}\right)\right. \\
+ & \frac{m_{3}^{2}}{\left(m_{1}^{2}-m_{2}^{2}\right)^{2}+m_{3}^{4}} \\
& \times\left[\mathrm{T}_{1134}\left(m_{1}^{2}, m_{1}^{2}, m_{2}^{2}, m_{2}^{2}, m_{2}^{2}, m_{1}^{2}, m_{3}^{2}\right)\right] \\
- & \left.\frac{m_{3}^{2}}{\left(m_{1}^{2}-m_{2}^{2}\right)^{2}+m_{3}^{4}} \mathrm{~T}_{1133}\left(m_{1}^{2}, m_{1}^{2}, m_{2}^{2}, m_{1}^{2}, m_{3}^{2}\right)\right] \\
+ & \frac{m_{1}^{4}\left(m_{1}^{2}-m_{2}^{2}\right)}{2\left[\left(m_{1}^{2}-m_{3}^{2}\right)+m_{2}^{2}\right)}\left(m_{2}^{2}-m_{1}^{2}+m_{3}^{2}\right) \\
& \times\left[\frac{\mathrm{T}_{1113}\left(m_{1}^{2}, m_{1}^{2}, m_{1}^{2}, m_{2}^{2}\right)}{m_{2}^{4}}+\frac{\mathrm{T}_{1113}\left(m_{2}^{2}, m_{2}^{2}, m_{2}^{2}, m_{1}^{2}\right)}{m_{1}^{4}}\right] \\
& -\frac{m_{2}^{2}-m_{1}^{2}+m_{3}^{2}}{\left(m_{1}^{2}-m_{2}^{2}\right)^{2}+m_{3}^{4}} \mathrm{~T}_{1114}\left(m_{1}^{2}, m_{1}^{2}, m_{1}^{2}, m_{3}^{2}\right) \\
- & \frac{m_{1}^{2}-m_{2}^{2}+m_{3}^{2}}{\left(m_{1}^{2}-m_{2}^{2}\right)^{2}+m_{3}^{4}} \mathrm{~T}_{1114}\left(m_{2}^{2}, m_{2}^{2}, m_{2}^{2}, m_{3}^{2}\right),
\end{aligned}
$$

$$
\begin{aligned}
\mathrm{T}_{11134}\left(m_{1}^{2}, m_{1}^{2}, m_{1}^{2}, m_{1}^{2}, 0\right)= & -\frac{3}{1+2 \epsilon} \mathrm{T}_{11113}\left(m_{1}^{2}, m_{1}^{2}, m_{1}^{2}, m_{1}^{2}, m_{1}^{2}\right), \\
\mathrm{T}_{11134}\left(m_{1}^{2}, m_{1}^{2}, m_{1}^{2}, m_{2}^{2}, m_{3}^{2}\right)= & \frac{\left(m_{2}^{2}-m_{1}^{2}+m_{3}^{2}\right) \epsilon}{\left[\left(m_{1}+m_{2}\right)^{2}-m_{3}^{2}\right]\left[\left(m_{1}-m_{2}\right)^{2}-m_{3}^{2}\right]} \mathrm{T}_{1134}\left(m_{1}^{2}, m_{1}^{2}, m_{2}^{2}, m_{3}^{2}\right) \\
& +\frac{m_{2}^{2}}{\left[\left(m_{1}+m_{2}\right)^{2}-m_{3}^{2}\right]\left[\left(m_{1}-m_{2}\right)^{2}-m_{3}^{2}\right]} \\
& \times\left[\mathrm{T}_{1134}\left(m_{1}^{2}, m_{1}^{2}, m_{2}^{2}, m_{3}^{2}\right)+\mathrm{T}_{1134}\left(m_{2}^{2}, m_{2}^{2}, m_{1}^{2}, m_{3}^{2}\right)\right] \\
& -\frac{m_{1}^{2}-m_{2}^{2}+m_{3}^{2}}{\left[\left(m_{1}+m_{2}\right)^{2}-m_{3}^{2}\right]\left[\left(m_{1}-m_{2}\right)^{2}-m_{3}^{2}\right]} \mathrm{T}_{1113}\left(m_{1}^{2}, m_{1}^{2}, m_{1}^{2}, m_{2}^{2}\right) \\
& -\frac{m_{1}^{2}+m_{2}^{2}-m_{3}^{2}}{\left[\left(m_{1}+m_{2}\right)^{2}-m_{3}^{2}\right]\left[\left(m_{1}-m_{2}\right)^{2}-m_{3}^{2}\right]} \mathrm{T}_{1114}\left(m_{1}^{2}, m_{1}^{2}, m_{1}^{2}, m_{3}^{2}\right) \\
& -\frac{1}{\left[\left(m_{1}+m_{2}\right)^{2}-m_{3}^{2}\right]\left[\left(m_{1}-m_{2}\right)^{2}-m_{3}^{2}\right]} \mathrm{T}_{113}\left(m_{1}^{2}, m_{1}^{2}, m_{2}^{2}\right),
\end{aligned}
$$

$$
\begin{aligned}
\mathrm{T}_{1134}\left(m_{1}^{2}, m_{1}^{2}, m_{1}^{2}, 0\right)= & \frac{1}{2 m_{1}^{2}} \mathrm{~T}_{113}\left(m_{1}^{2}, m_{1}^{2}, m_{1}^{2}\right), \\
\mathrm{T}_{1134}\left(m_{1}^{2}, m_{1}^{2}, m_{2}^{2}, m_{3}^{2}\right)= & \frac{\left(m_{2}^{2}-m_{1}^{2}+m_{3}^{2}\right)(-1+2 \epsilon)}{\left[\left(m_{1}+m_{2}\right)^{2}-m_{3}^{2}\right]\left[\left(m_{1}-m_{2}\right)^{2}-m_{3}^{2}\right]} \mathrm{T}_{134}\left(m_{1}^{2}, m_{2}^{2}, m_{3}^{2}\right) \\
& -\frac{m_{1}^{2}-m_{2}^{2}+m_{3}^{2}}{\left[\left(m_{1}+m_{2}\right)^{2}-m_{3}^{2}\right]\left[\left(m_{1}-m_{2}\right)^{2}-m_{3}^{2}\right]} \mathrm{T}_{113}\left(m_{1}^{2}, m_{1}^{2}, m_{2}^{2}\right) \\
& -\frac{m_{1}^{2}+m_{2}^{2}-m_{3}^{2}}{\left[\left(m_{1}+m_{2}\right)^{2}-m_{3}^{2}\right]\left[\left(m_{1}-m_{2}\right)^{2}-m_{3}^{2}\right]} \mathrm{T}_{114}\left(m_{1}^{2}, m_{1}^{2}, m_{3}^{2}\right) \\
& +\frac{2 m_{2}^{2}}{\left[\left(m_{1}+m_{2}\right)^{2}-m_{3}^{2}\right]\left[\left(m_{1}-m_{2}\right)^{2}-m_{3}^{2}\right]} \mathrm{T}_{334}\left(m_{2}^{2}, m_{2}^{2}, m_{3}^{2}\right) .
\end{aligned}
$$

Integrals with multiple denominators of the same loop-momentum structure and different masses are simplified by partial fractioning beforehand: 


$$
\begin{aligned}
\mathrm{T}_{a a \ldots}\left(m_{1}^{2}, m_{2}^{2}, \ldots\right)= & \frac{1}{m_{1}^{2}-m_{2}^{2}}\left[\mathrm{~T}_{a \ldots}\left(m_{1}^{2}, \ldots\right)-\mathrm{T}_{a \ldots}\left(m_{2}^{2}, \ldots\right)\right] \\
& \text { for } m_{1}^{2} \neq m_{2}^{2} \text { and } a \in\{1,3,4\} .
\end{aligned}
$$

All displayed integrals are symmetric under exchange of different loop-momentum structures:

$$
\begin{gathered}
\mathrm{T}_{a^{x} b^{y} \ldots}(\underbrace{m_{1}^{2}, \ldots, m_{1}^{2}}_{x}, \underbrace{m_{2}^{2}, \ldots, m_{2}^{2}}_{y}, \ldots)=\mathrm{T}_{b^{x} a^{y} \ldots}(\underbrace{m_{1}^{2}, \ldots, m_{1}^{2}}_{x}, \underbrace{m_{2}^{2}, \ldots, m_{2}^{2}}_{y}, \ldots) \\
\text { for } a, b \in\{1,3,4\}
\end{gathered}
$$

\section{Analytical $\mathcal{O}\left(\alpha_{t}^{2}\right)$ results}

The analytical expressions for the $\mathcal{O}\left(\alpha_{t}^{2}\right)$ contributions to the Higgs tadpoles and selfenergies that are described in section 2 are listed in the following.

\section{D.1 Symbols and abbreviations}

The following symbols and abbreviations are used to express the analytical results in a compact way. To shorten the notation the absolute-value bars of $\left|X_{t}\right|^{2},\left|Y_{t}\right|^{2}$ and $|\mu|^{2}$ are suppressed in the following terms:

$$
\begin{array}{rlrl}
\Delta_{a, b} & =m_{a}^{2}-m_{b}^{2}, & Y_{t} & =A_{t}^{*}+\mu t_{\beta}, \\
X_{t} & =A_{t}^{*}-\frac{\mu}{t_{\beta}}, & Y_{t}^{2} \equiv\left|Y_{t}\right|^{2}=Y_{t} Y_{t}^{*}, \quad y_{t}^{2}=\frac{Y_{t}^{2}}{\Delta_{\tilde{t}_{i} \tilde{t}_{j}}}, \\
X_{t}^{2} \equiv\left|X_{t}\right|^{2}=X_{t} X_{t}^{*}, \quad x_{t}^{2}=\frac{X_{t}^{2}}{\Delta_{\tilde{t}_{i} \tilde{t}_{j}}}, & \eta & =\frac{\mu^{2}}{s_{\beta}^{2} c_{\beta}^{2} \Delta_{\tilde{t}_{i} \tilde{t}_{j}}}-x_{t}^{2}-y_{t}^{2}, \\
\mu^{2} & \equiv|\mu|^{2}=\mu \mu^{*}, & U_{\times} & =\frac{1-U_{-}^{2}}{4}=\frac{m_{t}^{2}}{\Delta_{\tilde{t}_{i}} \tilde{t}_{j}} x_{t}^{2}, \\
U_{-} & =\mathbf{U}_{\tilde{t} 1 i} \mathbf{U}_{\tilde{t} 1 i}^{*}-\mathbf{U}_{\tilde{t} 1 j} \mathbf{U}_{\tilde{t} 1 j}^{*}, & \delta^{(1)} h_{t} & =h_{t}\left(\frac{\delta^{(1)} m_{t}}{m_{t}}-\frac{\delta^{(1)} M_{W}}{M_{W}}-\frac{\delta^{(1)} s_{\mathrm{w}}}{s_{\mathrm{w}}}-\frac{\delta^{(1)} s_{\beta}}{s_{\beta}}\right), \\
\frac{h_{t}}{\delta^{(1)} x_{t}^{2}} & =\frac{\delta^{(1)} X_{t}}{x_{t}^{2}}+\frac{\delta^{(1)} X_{t}^{*}}{X_{t}}, \quad \delta^{(1)} \phi_{X} & =-\frac{i}{2}\left(\frac{\delta^{(1)} X_{t}}{X_{t}}-\frac{\delta^{(1)} X_{t}^{*}}{X_{t}^{*}}\right), \\
\delta^{(1)} X_{t} & =\delta^{(1)} A_{t}^{*}-\frac{\delta^{(1)} \mu}{t_{\beta}}+\frac{\mu \delta^{(1)} t_{\beta}}{t_{\beta}^{2}}, & \delta^{(1)} X_{t}^{*} & =\delta^{(1)} A_{t}-\frac{\delta^{(1)} \mu^{*}}{t_{\beta}}+\frac{\mu^{*} \delta^{(1)} t_{\beta}}{t_{\beta}^{2}} .
\end{array}
$$

\section{D.2 Genuine two-loop self-energies}

The explicit expressions of the genuine two-loop integrals contributing to the Higgs-boson self-energies are depicted in the following.

$$
\begin{aligned}
\Sigma_{h h}^{(2) \text { gen }}=\frac{N_{c} s_{\beta}^{2} h_{t}^{4}}{256 \pi^{4}}\left\{s_{A}+4 m_{t}^{2} s_{B}\right\} & \begin{aligned}
+\sum_{\substack{i=1 \\
j \neq i}}^{2} \frac{N_{c} s_{\beta}^{2} h_{t}^{4}}{256 \pi^{4}}\{ & -x_{t}^{2}\left(1-12 U_{\times}\right) s_{D}-2 m_{t}^{2}\left(1+x_{t}^{2}\right)^{2} s_{E}+4 m_{t}^{2}\left(1+x_{t}^{2}\right) s_{F} \\
& -\left(1-16 U_{\times}\right) x_{t}^{2} s_{G}+\left(1-4 U_{\times}\right) x_{t}^{2} s_{H}+\left(1+x_{t}^{2}\left(1-4 U_{\times}\right)\right) s_{I_{1}} \\
& \left.+\left(1+x_{t}^{2}+4 U_{\times}\right) s_{I_{2}}+2\left(m_{t}^{2}-U_{\times} X_{t}^{2}\right) s_{J}+\frac{1}{2} s_{K_{1}}\right\}
\end{aligned}
\end{aligned}
$$




$$
\begin{aligned}
& \Sigma_{H H}^{(2) \text { gen }}= \frac{N_{c} c_{\beta}^{2} h_{t}^{4}}{256 \pi^{4}}\left\{s_{A}+4 m_{t}^{2} s_{B}\right\} \\
&+\sum_{\substack{i=1 \\
j \neq i}}^{2} \frac{N_{c} c_{\beta}^{2} h_{t}^{4}}{256 \pi^{4}}\left\{\frac{3 m_{t}^{2} \eta^{2}-Y_{t}^{2}}{\Delta_{\tilde{t}_{i} \tilde{t}_{j}}} s_{D}-\frac{m_{t}^{2}}{2}(\eta-2)^{2} s_{E}-2 m_{t}^{2}(\eta-2) s_{F}+\frac{4 m_{t}^{2} \eta^{2}-Y_{t}^{2}}{\Delta_{\tilde{t}_{i} \tilde{t}_{j}}} s_{G}\right. \\
& \quad-\frac{\left(m_{t}^{2} \eta^{2}-Y_{t}^{2}\right)}{\Delta_{\tilde{t}_{i} \tilde{t}_{j}}} s_{H}-\left(\frac{m_{t}^{2} \eta^{2}-Y_{t}^{2}}{\Delta_{\tilde{t}_{i} \tilde{t}_{j}}}-1\right) s_{I_{1}}-\left(\frac{2 m_{t}^{2} \eta-Y_{t}^{2}}{\Delta_{\tilde{t}_{i} \tilde{t}_{j}}}-1\right) s_{I_{2}} \\
& \\
&\left.\quad-m_{t}^{2}\left(\frac{\eta^{2}}{2}-2\right) s_{J}+\frac{1}{2} s_{L_{1}}\right\},
\end{aligned}
$$

$$
\begin{aligned}
& \Sigma_{h H}^{(2) \text { gen }}=-\frac{N_{c} s_{\beta} c_{\beta} h_{t}^{4}}{256 \pi^{4}}\left\{s_{A}+4 m_{t}^{2} s_{B}\right\} \\
& -\sum_{\substack{i=1 \\
j \neq i}}^{2} \frac{N_{c} s_{\beta} c_{\beta} h_{t}^{4}}{256 \pi^{4}}\left\{-\frac{\eta}{2}\left(1-12 U_{\times}\right) s_{D}+m_{t}^{2}(\eta-2)\left(1+x_{t}^{2}\right) s_{E}\right. \\
& -2 m_{t}^{2}\left[\frac{\eta}{2}-1-\left(1+x_{t}^{2}\right)\right] s_{F}+\left(1-16 U_{\times}\right) \frac{\eta}{2} s_{G}+\left(1-4 U_{\times}\right) \frac{\eta}{2} s_{H} \\
& +\left[1-\frac{\eta}{2}\left(1-4 U_{\times}\right)\right] s_{I_{1}}+\left[1+2 U_{\times}-\frac{\eta}{2}\left(1+\frac{2 m_{t}^{2}}{\Delta_{\tilde{t}_{i} \tilde{t}_{j}}}\right)\right] s_{I_{2}} \\
& \left.+m_{t}^{2}\left(\eta x_{t}^{2}+2\right) s_{J}+\frac{1}{2} s_{K_{1}}+\frac{1}{2}\left(\frac{\eta}{2}+x_{t}^{2}\right) s_{K_{2}}-s_{K_{3}}\right\} \text {, } \\
& \Sigma_{A A}^{(2) \text { gen }}=\frac{N_{c} c_{\beta}^{2} h_{t}^{4}}{256 \pi^{4}} s_{A}
\end{aligned}
$$$$
\begin{aligned}
+\sum_{\substack{i=1 \\
j \neq i}}^{2} \frac{N_{c} c_{\beta}^{2} h_{t}^{4}}{256 \pi^{4}}\{ & -\frac{3 m_{t}^{2} \eta^{2}+\left(1-12 U_{\times}\right) Y_{t}^{2}}{\Delta_{\tilde{t}_{i} \tilde{t}_{j}}} s_{D}+\frac{m_{t}^{2}}{2}\left(\eta^{2}-4 x_{t}^{2} y_{t}^{2}\right) s_{E}-\frac{4 m_{t}^{2} \eta^{2}+\left(1-16 U_{\times}\right) Y_{t}^{2}}{\Delta_{\tilde{t}_{i} \tilde{t}_{j}}} s_{G} \\
& +\frac{m_{t}^{2} \eta^{2}+\left(1-4 U_{\times}\right) Y_{t}^{2}}{\Delta_{\tilde{t}_{i} \tilde{t}_{j}}} s_{H}+\left[1+\frac{m_{t}^{2} \eta^{2}}{\Delta_{\tilde{t}_{i} \tilde{t}_{j}}}+\left(1-4 U_{\times}\right) y_{t}^{2}\right] s_{I_{1}} \\
& \left.+\left(1+y_{t}^{2}\right) s_{I_{2}}+\left(\frac{m_{t}^{2} \eta^{2}}{2}-2 U_{\times} Y_{t}^{2}\right) s_{J}+\frac{1}{2} s_{L_{1}}+\eta s_{L_{2}}-s_{L_{3}}\right\}
\end{aligned}
$$

$$
\begin{aligned}
& \Sigma_{h A}^{(2) \text { gen }}=\sum_{\substack{i=1 \\
j \neq i}}^{2} \frac{N_{c} h_{t}^{4}}{256 \pi^{4}} \frac{\Im \mathfrak{m}\left[X_{t} \mu^{*}\right]}{s_{\beta} c_{\beta} \Delta_{\tilde{t}_{i} \tilde{t}_{j}}}\left\{\left(1-12 U_{\times}\right) s_{D}+2 m_{t}^{2}\left(1+x_{t}^{2}\right) s_{E}-2 m_{t}^{2} s_{F}+\left(1-16 U_{\times}\right) s_{G}\right. \\
& \left.-\left(1-4 U_{\times}\right)\left(s_{H}+s_{I_{1}}\right)-\left(1+\frac{2 m_{t}^{2}}{\Delta_{\tilde{t}_{i} \tilde{t}_{j}}}\right) s_{I_{2}}+2 m_{t}^{2} x_{t}^{2} s_{J}+\frac{1}{2} s_{K_{2}}\right\},
\end{aligned}
$$

$$
\Sigma_{H A}^{(2) \text { gen }}=\sum_{\substack{i=1 \\ j \neq i}}^{2} \frac{N_{c} c_{\beta} h_{t}^{4}}{256 \pi^{4} s_{\beta}} \frac{\Im \mathfrak{m}\left[X_{t} \mu^{*}\right]}{s_{\beta} c_{\beta} \Delta_{\tilde{t}_{i} \tilde{t}_{j}}}\left\{-\frac{6 m_{t}^{2} \eta}{\Delta_{\tilde{t}_{i} \tilde{t}_{j}}} s_{D}+m_{t}^{2}(\eta-2) s_{E}+2 m_{t}^{2} s_{F}-\frac{8 m_{t}^{2} \eta}{\Delta_{\tilde{t}_{i} \tilde{t}_{j}}} s_{G}\right.
$$

$$
\left.+\frac{2 m_{t}^{2} \eta}{\Delta_{\tilde{t}_{i} \tilde{t}_{j}}} s_{H}+\frac{2 m_{t}^{2} \eta}{\Delta_{\tilde{t}_{i} \tilde{t}_{j}}} s_{I_{1}}+\frac{2 m_{t}^{2}}{\Delta_{\tilde{t}_{i} \tilde{t}_{j}}} s_{I_{2}}+m_{t}^{2} \eta s_{J}+s_{L_{2}}\right\}
$$

$\Sigma_{H^{ \pm} H^{ \pm}}^{(2) \text { gen }}=\frac{N_{c} c_{\beta}^{2} h_{t}^{4}}{256 \pi^{4}}\left\{s_{A}+\frac{\mu^{2}}{s_{\beta}^{2} c_{\beta}^{2}} \frac{m_{t}^{2}}{\Delta_{\tilde{t}_{i} \tilde{b}_{1}} \Delta_{\tilde{t}_{j} \tilde{b}_{1}}} s_{C}\right\}$

$$
\begin{gathered}
+\sum_{\substack{i=1 \\
j \neq i}}^{2} \frac{N_{c} c_{\beta}^{2} h_{t}^{4}}{256 \pi^{4}}\left\{\frac{\Delta_{\tilde{t}_{i} \tilde{t}_{j}}}{2 \Delta_{\tilde{t}_{i} \tilde{b}_{1}}}\left[\left(y_{t}^{2}+1\right)\left(\frac{2 m_{t}^{2}}{\Delta_{\tilde{t}_{i} \tilde{t}_{j}}}+1-U_{-}\right)-\frac{2 \mu^{2} m_{t}^{2}}{c_{\beta}^{2} s_{\beta}^{2} \Delta_{\tilde{t}_{i} \tilde{t}_{j}}^{2}}\right]\left(s_{I_{1}}+s_{I_{2}}\right)\right. \\
\left.+\frac{m_{t}^{2} \mu^{2} \Delta_{\tilde{t}_{i} \tilde{t}_{j}}}{2 s_{\beta}^{2} c_{\beta}^{2} \Delta_{\tilde{t}_{i} \tilde{b}_{1}} \Delta_{\tilde{t}_{j} \tilde{b}_{1}}} s_{M}+s_{N}+\frac{\Delta_{\tilde{t}_{i} \tilde{t}_{j}}}{\Delta_{\tilde{t}_{i} \tilde{b}_{1}}} s_{O}\right\} .
\end{gathered}
$$




$$
\begin{aligned}
& s_{A}=\mathrm{T}_{134}\left(m_{t}^{2}, m_{\tilde{b}_{1}}^{2}, \mu^{2}\right)-3 \mathrm{~T}_{113}\left(m_{t}^{2}, m_{t}^{2}, \mu^{2}\right)-2 \mathrm{~T}_{113}\left(m_{t}^{2}, m_{t}^{2}, m_{t}^{2}\right)+\mathrm{T}_{113}\left(m_{t}^{2}, m_{t}^{2}, m_{\tilde{b}_{1}}^{2}\right) \\
& +\left(m_{t}^{2}-m_{\tilde{b}_{1}}^{2}+\mu^{2}\right) \mathrm{T}_{1134}\left(m_{t}^{2}, m_{t}^{2}, m_{\tilde{b}_{1}}^{2}, \mu^{2}\right)+\sum_{i=1}^{2} \mathrm{~T}_{113}\left(m_{t}^{2}, m_{t}^{2}, m_{\tilde{t}_{i}}^{2}\right) \\
& +c_{\beta}^{2} s_{A}^{H}+\left.s_{\beta}^{2} s_{A}^{H}\right|_{m_{H^{ \pm}} \rightarrow 0, Y_{t}^{2} \rightarrow X_{t}^{2}, y_{t}^{2} \rightarrow x_{t}^{2}, \eta \rightarrow-2 x_{t}^{2}}, \\
& s_{A}^{H}=3 \mathrm{~T}_{113}\left(m_{t}^{2}, m_{t}^{2}, m_{H^{ \pm}}^{2}\right)+\mathrm{T}_{134}\left(m_{t}^{2}, 0, m_{H^{ \pm}}^{2}\right)+2 \mathrm{~T}_{134}\left(m_{t}^{2}, m_{t}^{2}, m_{H^{ \pm}}^{2}\right) \\
& +\left(m_{t}^{2}-m_{H^{ \pm}}^{2}\right) \mathrm{T}_{1134}\left(m_{t}^{2}, m_{t}^{2}, 0, m_{H^{ \pm}}^{2}\right)+2\left(2 m_{t}^{2}-m_{H^{ \pm}}^{2}\right) \mathrm{T}_{1134}\left(m_{t}^{2}, m_{t}^{2}, m_{t}^{2}, m_{H^{ \pm}}^{2}\right), \\
& s_{B}=\mathrm{T}_{1113}\left(m_{t}^{2}, m_{t}^{2}, m_{t}^{2}, m_{\tilde{b}_{1}}^{2}\right)-3 \mathrm{~T}_{1113}\left(m_{t}^{2}, m_{t}^{2}, m_{t}^{2}, \mu^{2}\right)-2 \mathrm{~T}_{1113}\left(m_{t}^{2}, m_{t}^{2}, m_{t}^{2}, m_{t}^{2}\right) \\
& -\mathrm{T}_{1133}\left(m_{t}^{2}, m_{t}^{2}, m_{t}^{2}, m_{t}^{2}\right)+\mathrm{T}_{1134}\left(m_{t}^{2}, m_{t}^{2}, m_{\tilde{b}_{1}}^{2}, \mu^{2}\right) \\
& +\left(m_{t}^{2}-m_{\tilde{b}_{1}}^{2}+\mu^{2}\right) \mathrm{T}_{11134}\left(m_{t}^{2}, m_{t}^{2}, m_{t}^{2}, m_{\tilde{b}_{1}}^{2}, \mu^{2}\right) \\
& +\sum_{i=1}^{2}\left[\left(m_{t}^{2}-m_{\tilde{t}_{i}}^{2}+\mu^{2}\right) \mathrm{T}_{11134}\left(m_{t}^{2}, m_{t}^{2}, m_{t}^{2}, m_{\tilde{t}_{i}}^{2}, \mu^{2}\right)+\mathrm{T}_{1113}\left(m_{t}^{2}, m_{t}^{2}, m_{t}^{2}, m_{\tilde{t}_{i}}^{2}\right)\right] \\
& +c_{\beta}^{2} s_{B}^{H}+\left.s_{\beta}^{2} s_{B}^{H}\right|_{m_{H^{ \pm}} \rightarrow 0, Y_{t}^{2} \rightarrow X_{t}^{2}, y_{t}^{2} \rightarrow x_{t}^{2}, \eta \rightarrow-2 x_{t}^{2}}, \\
& s_{B}^{H}=3 \mathrm{~T}_{1113}\left(m_{t}^{2}, m_{t}^{2}, m_{t}^{2}, m_{H^{ \pm}}^{2}\right)+\left(m_{t}^{2}-m_{H^{ \pm}}^{2}\right) \mathrm{T}_{11134}\left(m_{t}^{2}, m_{t}^{2}, m_{t}^{2}, 0, m_{H^{ \pm}}^{2}\right) \\
& +\mathrm{T}_{1134}\left(m_{t}^{2}, m_{t}^{2}, 0, m_{H^{ \pm}}^{2}\right)+4 \mathrm{~T}_{1134}\left(m_{t}^{2}, m_{t}^{2}, m_{t}^{2}, m_{H^{ \pm}}^{2}\right) \\
& +\left(2 m_{t}^{2}-m_{H^{ \pm}}^{2}\right)\left(2 \mathrm{~T}_{11134}\left(m_{t}^{2}, m_{t}^{2}, m_{t}^{2}, m_{t}^{2}, m_{H^{ \pm}}^{2}\right)+\mathrm{T}_{11334}\left(m_{t}^{2}, m_{t}^{2}, m_{t}^{2}, m_{t}^{2}, m_{H^{ \pm}}^{2}\right)\right) \text {, } \\
& s_{C}=-\mathrm{T}_{113}\left(m_{\tilde{b}_{1}}^{2}, m_{\tilde{b}_{1}}^{2}, m_{t}^{2}\right)-\mathrm{T}_{113}\left(m_{\tilde{b}_{1}}^{2}, m_{\tilde{b}_{1}}^{2}, \mu^{2}\right)+\mathrm{T}_{134}\left(m_{t}^{2}, m_{\tilde{b}_{1}}^{2}, \mu^{2}\right) \\
& -\left(m_{t}^{2}-m_{\tilde{b}_{1}}^{2}+\mu^{2}\right) \mathrm{T}_{1134}\left(m_{\tilde{b}_{1}}^{2}, m_{\tilde{b}_{1}}^{2}, m_{t}^{2}, \mu^{2}\right) \\
& +\frac{m_{t}^{2}}{\Delta_{\tilde{t}_{i} \tilde{b}_{1}} \Delta_{\tilde{t}_{j} \tilde{b}_{1}}}\left[\mathrm{~T}_{13}\left(m_{\tilde{b}_{1}}^{2}, \mu^{2}\right)-4 \mathrm{~T}_{13}\left(m_{\tilde{b}_{1}}^{2}, m_{\tilde{b}_{1}}^{2}\right)-\left(m_{\tilde{b}_{1}}^{2}-\mu^{2}\right) \mathrm{T}_{134}\left(m_{\tilde{b}_{1}}^{2}, \mu^{2}, 0\right)\right] \\
& +c_{\beta}^{2}\left\{\mathrm{~T}_{113}\left(m_{\tilde{b}_{1}}^{2}, m_{\tilde{b}_{1}}^{2}, m_{H^{ \pm}}^{2}\right)-\frac{m_{t}^{2}}{\Delta_{\tilde{t}_{i} \tilde{b}_{1}} \Delta_{\tilde{t}_{j} \tilde{b}_{1}}}\left[\mathrm{~T}_{13}\left(m_{\tilde{b}_{1}}^{2}, m_{H^{ \pm}}^{2}\right)+\mu^{2} \mathrm{~T}_{134}\left(m_{\tilde{b}_{1}}^{2}, m_{\tilde{b}_{1}}^{2}, m_{H^{ \pm}}^{2}\right)\right]\right\}, \\
& s_{D}=\frac{U_{-}}{\Delta_{\tilde{t}_{i} \tilde{t}_{j}}}\left[\mathrm{~T}_{13}\left(m_{\tilde{t}_{i}}^{2}, m_{\tilde{b}_{1}}^{2}\right)-\mathrm{T}_{13}\left(m_{\tilde{t}_{i}}^{2}, \mu^{2}\right)+c_{\beta}^{2} \mathrm{~T}_{13}\left(m_{\tilde{t}_{i}}^{2}, m_{H^{ \pm}}^{2}\right)\right], \\
& s_{E}=\left(1-U_{-}\right) \mathrm{T}_{1113}\left(m_{\tilde{t}_{i}}^{2}, m_{\tilde{t}_{i}}^{2}, m_{\tilde{t}_{i}}^{2}, m_{\tilde{b}_{1}}^{2}\right)-2\left(8 U_{\times}-1\right) \mathrm{T}_{1113}\left(m_{\tilde{t}_{i}}^{2}, m_{\tilde{t}_{i}}^{2}, m_{\tilde{t}_{i}}^{2}, m_{\tilde{t}_{j}}^{2}\right) \\
& -2\left(m_{t}^{2}-m_{\tilde{t}_{i}}^{2}+\mu^{2}\right) \mathrm{T}_{11134}\left(m_{\tilde{t}_{i}}^{2}, m_{\tilde{t}_{i}}^{2}, m_{\tilde{t}_{i}}^{2}, m_{t}^{2}, \mu^{2}\right)+\left(U_{-}-3\right) \mathrm{T}_{1113}\left(m_{\tilde{t}_{i}}^{2}, m_{\tilde{t}_{i}}^{2}, m_{\tilde{t}_{i}}^{2}, \mu^{2}\right) \\
& +\left(1-U_{-}\right)\left(m_{\tilde{t}_{i}}^{2}-\mu^{2}\right) \mathrm{T}_{11134}\left(m_{\tilde{t}_{i}}^{2}, m_{\tilde{t}_{i}}^{2}, m_{\tilde{t}_{i}}^{2}, 0, \mu^{2}\right)+16 U_{\times} \mathrm{T}_{1113}\left(m_{\tilde{t}_{i}}^{2}, m_{\tilde{t}_{i}}^{2}, m_{\tilde{t}_{i}}^{2}, m_{\tilde{t}_{i}}^{2}\right) \\
& +8 U_{\times} \mathrm{T}_{1133}\left(m_{\tilde{t}_{i}}^{2}, m_{\tilde{t}_{i}}^{2}, m_{\tilde{t}_{i}}^{2}, m_{\tilde{t}_{i}}^{2}\right)-2 \mathrm{~T}_{1113}\left(m_{\tilde{t}_{i}}^{2}, m_{\tilde{t}_{i}}^{2}, m_{\tilde{t}_{i}}^{2}, m_{t}^{2}\right) \\
& +c_{\beta}^{2} s_{E}^{H}+\left.s_{\beta}^{2} s_{E}^{H}\right|_{m_{H^{ \pm}} \rightarrow 0, Y_{t}^{2} \rightarrow X_{t}^{2}, y_{t}^{2} \rightarrow x_{t}^{2}, \eta \rightarrow-2 x_{t}^{2}}, \\
& s_{E}^{H}=2 m_{t}^{2}\left(1+x_{t}^{2} y_{t}^{2}-\eta\right)\left[2 \mathrm{~T}_{11134}\left(m_{\tilde{t}_{i}}^{2}, m_{\tilde{t}_{i}}^{2}, m_{\tilde{t}_{i}}^{2}, m_{\tilde{t}_{i}}^{2}, m_{H^{ \pm}}^{2}\right)+\mathrm{T}_{11334}\left(m_{\tilde{t}_{i}}^{2}, m_{\tilde{t}_{i}}^{2}, m_{\tilde{t}_{i}}^{2}, m_{\tilde{t}_{i}}^{2}, m_{H^{ \pm}}^{2}\right)\right] \\
& +2 Y_{t}^{2}\left(1-2 U_{\times}\right) \mathrm{T}_{11134}\left(m_{\tilde{t}_{i}}^{2}, m_{\tilde{t}_{i}}^{2}, m_{\tilde{t}_{i}}^{2}, m_{\tilde{t}_{j}}^{2}, m_{H^{ \pm}}^{2}\right)+\left(3-U_{-}\right) \mathrm{T}_{1113}\left(m_{\tilde{t}_{i}}^{2}, m_{\tilde{t}_{i}}^{2}, m_{\tilde{t}_{i}}^{2}, m_{H^{ \pm}}^{2}\right) \\
& +\left(m_{t}^{2}\left(1+U_{-}-2 \eta\right)+\left(1-U_{-}\right) Y_{t}^{2}\right) \mathrm{T}_{11134}\left(m_{\tilde{t}_{i}}^{2}, m_{\tilde{t}_{i}}^{2}, m_{\tilde{t}_{i}}^{2}, m_{\tilde{b}_{1}}^{2}, m_{H^{ \pm}}^{2}\right), \\
& s_{F}=\left(m_{t}^{2}-m_{\tilde{t}_{i}}^{2}+\mu^{2}\right) \mathrm{T}_{11334}\left(m_{\tilde{t}_{i}}^{2}, m_{\tilde{t}_{i}}^{2}, m_{t}^{2}, m_{t}^{2}, \mu^{2}\right)+\mathrm{T}_{1133}\left(m_{\tilde{t}_{i}}^{2}, m_{\tilde{t}_{i}}^{2}, m_{t}^{2}, m_{t}^{2}\right), \\
& s_{G}=\frac{1-4 U_{\times}}{\Delta_{\tilde{t}_{i} \tilde{t}_{j}}}\left[4 \mathrm{~T}_{13}\left(m_{\tilde{t}_{i}}^{2}, m_{\tilde{t}_{i}}^{2}\right)-4 \mathrm{~T}_{13}\left(m_{\tilde{t}_{i}}^{2}, m_{\tilde{t}_{j}}^{2}\right)\right. \\
& \left.-c_{\beta}^{2} Y_{t}^{2} \mathrm{~T}_{134}\left(m_{\tilde{t}_{i}}^{2}, m_{\tilde{t}_{j}}^{2}, m_{H^{ \pm}}^{2}\right)-s_{\beta}^{2} X_{t}^{2} \mathrm{~T}_{134}\left(m_{\tilde{t}_{i}}^{2}, m_{\tilde{t}_{j}}^{2}, 0\right)\right], \\
& s_{H}=-\mathrm{T}_{134}\left(m_{\tilde{t}_{i}}^{2}, m_{t}^{2}, \mu^{2}\right) \text {, }
\end{aligned}
$$




$$
\begin{aligned}
& s_{I_{1}}=-\frac{1}{2}\left(1-3 U_{-}\right) \mathrm{T}_{113}\left(m_{\tilde{t}_{i}}^{2}, m_{\tilde{t}_{i}}^{2}, m_{\tilde{b}_{1}}^{2}\right)+\left(m_{t}^{2}-m_{\tilde{t}_{j}}^{2}+\mu^{2}\right) \mathrm{T}_{1134}\left(m_{\tilde{t}_{i}}^{2}, m_{\tilde{t}_{i}}^{2}, m_{t}^{2}, \mu^{2}\right) \\
& -\left(9-40 U_{\times}\right) \mathrm{T}_{113}\left(m_{\tilde{t}_{i}}^{2}, m_{\tilde{t}_{i}}^{2}, m_{\tilde{t}_{j}}^{2}\right)+\frac{3}{2}\left(1-U_{-}\right) \mathrm{T}_{113}\left(m_{\tilde{t}_{i}}^{2}, m_{\tilde{t}_{i}}^{2}, \mu^{2}\right) \\
& +8\left(1-5 U_{\times}\right) \mathrm{T}_{113}\left(m_{\tilde{t}_{i}}^{2}, m_{\tilde{t}_{i}}^{2}, m_{\tilde{t}_{i}}^{2}\right)+\mathrm{T}_{113}\left(m_{\tilde{t}_{i}}^{2}, m_{\tilde{t}_{i}}^{2}, m_{t}^{2}\right) \\
& +c_{\beta}^{2} s_{I_{1}}^{H}+\left.s_{\beta}^{2} s_{I_{1}}^{H}\right|_{m_{H^{ \pm}} \rightarrow 0, Y_{t}^{2} \rightarrow X_{t}^{2}, y_{t}^{2} \rightarrow x_{t}^{2}, \eta \rightarrow-2 x_{t}^{2}}, \\
& s_{I_{1}}^{H}=-Y_{t}^{2}\left(3-10 U_{\times}\right) \mathrm{T}_{1134}\left(m_{\tilde{t}_{i}}^{2}, m_{\tilde{t}_{i}}^{2}, m_{\tilde{t}_{j}}^{2}, m_{H^{ \pm}}^{2}\right)-\frac{3}{2}\left(1-U_{-}\right) \mathrm{T}_{113}\left(m_{\tilde{t}_{i}}^{2}, m_{\tilde{t}_{i}}^{2}, m_{H^{ \pm}}^{2}\right) \text {, } \\
& s_{I_{2}}=U_{-}\left[\mathrm{T}_{113}\left(m_{\tilde{t}_{i}}^{2}, m_{\tilde{t}_{i}}^{2}, \mu^{2}\right)-\mathrm{T}_{113}\left(m_{\tilde{t}_{i}}^{2}, m_{\tilde{t}_{i}}^{2}, m_{\tilde{b}_{1}}^{2}\right)\right]-\Delta_{\tilde{t}_{i} \tilde{t}_{j}} \mathrm{~T}_{1134}\left(m_{\tilde{t}_{i}}^{2}, m_{\tilde{t}_{i}}^{2}, m_{t}^{2}, \mu^{2}\right) \\
& +8\left(1-4 U_{\times}\right)\left[\mathrm{T}_{113}\left(m_{\tilde{t}_{i}}^{2}, m_{\tilde{t}_{i}}^{2}, m_{\tilde{t}_{j}}^{2}\right)-\mathrm{T}_{113}\left(m_{\tilde{t}_{i}}^{2}, m_{\tilde{t}_{i}}^{2}, m_{\tilde{t}_{i}}^{2}\right)\right] \\
& +c_{\beta}^{2} s_{I_{2}}^{H}+\left.s_{\beta}^{2} s_{I_{2}}^{H}\right|_{m_{H} \pm 0, Y_{t}^{2} \rightarrow X_{t}^{2}, y_{t}^{2} \rightarrow x_{t}^{2}, \eta \rightarrow-2 x_{t}^{2}}, \\
& s_{I_{2}}^{H}=2 Y_{t}^{2}\left(1-4 U_{\times}\right) T_{1134}\left(m_{\tilde{t}_{i}}^{2}, m_{\tilde{t}_{i}}^{2}, m_{\tilde{t}_{j}}^{2}, m_{H^{ \pm}}^{2}\right)-U_{-} \mathrm{T}_{113}\left(m_{\tilde{t}_{i}}^{2}, m_{\tilde{t}_{i}}^{2}, m_{H^{ \pm}}^{2}\right) \text {, } \\
& s_{J}=-\left(1-8 U_{\times}\right) \mathrm{T}_{1133}\left(m_{\tilde{t}_{i}}^{2}, m_{\tilde{t}_{i}}^{2}, m_{\tilde{t}_{j}}^{2}, m_{\tilde{t}_{j}}^{2}\right)-c_{\beta}^{2} s_{J}^{H}-\left.s_{\beta}^{2} s_{J}^{H}\right|_{m_{H^{ \pm}} \rightarrow 0, Y_{t}^{2} \rightarrow X_{t}^{2}, y_{t}^{2} \rightarrow x_{t}^{2}, \eta \rightarrow-2 x_{t}^{2}}, \\
& s_{J}^{H}=Y_{t}^{2}\left(1-2 U_{\times}\right) \mathrm{T}_{11334}\left(m_{\tilde{t}_{i}}^{2}, m_{\tilde{t}_{i}}^{2}, m_{\tilde{t}_{j}}^{2}, m_{\tilde{t}_{j}}^{2}, m_{H^{ \pm}}^{2}\right), \\
& s_{K_{1}}=\left\{U_{-}\left[\left(x_{t}^{2}\left(2 \frac{\mu^{2}-m_{\tilde{t}_{i}}^{2}}{\Delta_{\tilde{t}_{i} \tilde{t}_{j}}}+1\right)\left(1-12 U_{\times}\right)\right)+1-8 U_{\times}\right]-x_{t}^{2}\left(1-4 U_{\times}\right)-1\right\} \mathrm{T}_{134}\left(m_{\tilde{t}_{i}}^{2}, \mu^{2}, 0\right) \\
& +\left[-8 U_{\times} \Delta_{\tilde{t}_{i} \tilde{t}_{j}}+2 m_{t}^{2}+2\left(\mu^{2}-m_{\tilde{t}_{i}}^{2}\right)\right] \mathrm{T}_{1134}\left(m_{t}^{2}, m_{t}^{2}, m_{\tilde{t}_{i}}^{2}, \mu^{2}\right) \\
& -\left\{\left(\mu^{2}-m_{\tilde{t}_{i}}^{2}\right)\left[\left(1-4 U_{\times}\right)+1-U_{-}\left(x_{t}^{2}\left(1-12 U_{\times}\right)+1-8 U_{\times}\right)\right]\right. \\
& \left.+4\left(1-U_{-}\right) m_{t}^{2}\left(x_{t}^{2}+1\right)^{2}\right\} \mathrm{T}_{1134}\left(m_{\tilde{t}_{i}}^{2}, m_{\tilde{t}_{i}}^{2}, 0, \mu^{2}\right) \\
& +c_{\beta}^{2} s_{K_{1}}^{H}+\left.s_{\beta}^{2} s_{K_{1}}^{H}\right|_{m_{H^{ \pm}} \rightarrow 0, Y_{t}^{2} \rightarrow X_{t}^{2}, y_{t}^{2} \rightarrow x_{t}^{2}, \eta \rightarrow-2 x_{t}^{2}}, \\
& s_{K_{1}}^{H}=\left[-1+\left(2 \eta-U_{-}\right)\left(1-6 U_{\times}\right)\left(1-4 U_{\times}\right)-2 U_{-} x_{t}^{2} y_{t}^{2}\left(1-12 U_{\times}\right)\right] \mathrm{T}_{134}\left(m_{\tilde{t}_{i}}^{2}, m_{\tilde{b}_{1}}^{2}, m_{H^{ \pm}}^{2}\right) \\
& +2\left\{-1+\left[1-4 U_{\times}\right]\left[\eta\left(1-6 U_{\times}\right)-x_{t}^{2} y_{t}^{2}\left(1-16 U_{\times}\right)\right]\right\} \mathrm{T}_{134}\left(m_{\tilde{t}_{i}}^{2}, m_{\tilde{t}_{i}}^{2}, m_{H^{ \pm}}^{2}\right) \\
& +\left\{\left(\eta-\frac{U_{-}}{2}\right)\left[\frac{2 m_{t}^{2}}{\Delta_{\tilde{t}_{i} \tilde{t}_{j}}}\left(5-8 U_{\times}\right)+2 U_{\times}\left(5-12 U_{\times}\right)\right]+\left[1-4 U_{\times}\right]\left[1-U_{\times}-x_{t}^{2} y_{t}^{2}\right]-\frac{5 m_{t}^{2}}{\Delta_{\tilde{t}_{i} \tilde{t}_{j}}}\right. \\
& \left.+U_{-}\left[x_{t}^{2} y_{t}^{2}\left(1-12 U_{\times}\right)+y_{t}^{2}\left(1-8 U_{\times}\right)\right]-1-y_{t}^{2}\right\} \Delta_{\tilde{t}_{i} \tilde{t}_{j}} \mathrm{~T}_{1134}\left(m_{\tilde{t}_{i}}^{2}, m_{\tilde{t}_{i}}^{2}, m_{\tilde{b}_{1}}^{2}, m_{H^{ \pm}}^{2}\right) \\
& +4 m_{t}^{2}\left\{\eta\left[x_{t}^{2}\left(5-12 U_{\times}\right)+5-8 U_{\times}\right]+x_{t}^{2} y_{t}^{2}\left[-\left(5 x_{t}^{2}\left(1-4 U_{\times}\right)+5-16 U_{\times}\right)\right]\right. \\
& \left.-x_{t}^{2}\left[5-4 U_{\times}\right]-5\right\} \mathrm{T}_{1134}\left(m_{\tilde{t}_{i}}^{2}, m_{\tilde{t}_{i}}^{2}, m_{\tilde{t}_{i}}^{2}, m_{H^{ \pm}}^{2}\right) \text {, } \\
& s_{K_{2}}=8\left\{\left(\mu^{2}-m_{\tilde{t}_{i}}^{2}\right)\left(\frac{U_{-} m_{t}^{2}\left(x_{t}^{2}+\frac{1}{2}\right)}{\Delta_{\tilde{t}_{i} \tilde{t}_{j}}}+\frac{\left(1-U_{-}\right)\left(1-4 U_{\times}\right)}{8}\right)-\frac{m_{t}^{2}\left(x_{t}^{2}+1\right)\left(1-U_{-}\right)}{2}\right\} \mathrm{T}_{1134}\left(m_{\tilde{t}_{i}}^{2}, m_{\tilde{t}_{i}}^{2}, \mu^{2}, 0\right) \\
& +\left\{-U_{-}\left[\left(1-12 U_{\times}\right)\left(2 \frac{\mu^{2}-m_{\tilde{t}_{i}}^{2}}{\Delta_{\tilde{t}_{i} \tilde{t}_{j}}}+1\right)-\frac{4 m_{t}^{2}}{\Delta_{\tilde{t}_{i} \tilde{t}_{j}}}\right]+1-4 U_{\times}\right\} \mathrm{T}_{134}\left(m_{\tilde{t}_{i}}^{2}, \mu^{2}, 0\right) \\
& +4 m_{t}^{2} \mathrm{~T}_{1134}\left(m_{t}^{2}, m_{t}^{2}, m_{\tilde{t}_{i}}^{2}, \mu^{2}\right)+c_{\beta}^{2} s_{K_{2}}^{H}+\left.s_{\beta}^{2} s_{K_{2}}^{H}\right|_{m_{H^{ \pm}} \rightarrow 0, Y_{t}^{2} \rightarrow X_{t}^{2}, y_{t}^{2} \rightarrow x_{t}^{2}, \eta \rightarrow-2 x_{t}^{2}},
\end{aligned}
$$




$$
\begin{aligned}
& s_{K_{2}}^{H}=\left\{2\left[1-4 U_{\times}\right]\left[1+\frac{3 m_{t}^{2}}{\Delta_{\tilde{t}_{i} \tilde{t}_{j}}}\left(2 \eta-U_{-} m_{t}^{2}\right)\right]+2 U_{-} y_{t}^{2}\left[1-12 U_{\times}\right]\right\} \mathrm{T}_{134}\left(m_{\tilde{t}_{i}}^{2}, m_{\tilde{b}_{1}}^{2}, m_{H^{ \pm}}^{2}\right) \\
& +2\left(1-4 U_{\times}\right)\left[1+\frac{6 \eta m_{t}^{2}}{\Delta_{\tilde{t}_{i} \tilde{t}_{j}}}+y_{t}^{2}\left(1-16 U_{\times}\right)\right] \mathrm{T}_{134}\left(m_{\tilde{t}_{i}}^{2}, m_{\tilde{t}_{i}}^{2}, m_{H^{ \pm}}^{2}\right) \\
& +\left\{m_{t}^{2}\left[4 x_{t}^{2}+7-4 U_{\times}\right]+\left[\frac{4 m_{t}^{2}}{\Delta_{\tilde{t}_{i} \tilde{t}_{j}}}-3\left(1-4 U_{\times}\right)\right]\left[2 \eta m_{t}^{2}+U_{-}\left(Y_{t}^{2}-m_{t}^{2}\right)\right]\right. \\
& \left.+Y_{t}^{2}\left[2 U_{-}+1-4 U_{\times}\right]\right\} \mathrm{T}_{1134}\left(m_{\tilde{t}_{i}}^{2}, m_{\tilde{t}_{i}}^{2}, m_{\tilde{b}_{1}}^{2}, m_{H^{ \pm}}^{2}\right) \\
& +4 m_{t}^{2}\left[\frac{4 \eta m_{t}^{2}}{\Delta_{\tilde{t}_{i} \tilde{t}_{j}}}+2 x_{t}^{2}+4+\left(1-4 U_{\times}\right)\left(1-3 \eta+2 y_{t}^{2}+5 x_{t}^{2} y_{t}^{2}\right)\right] \mathrm{T}_{1134}\left(m_{\tilde{t}_{i}}^{2}, m_{\tilde{t}_{i}}^{2}, m_{\tilde{t}_{i}}^{2}, m_{H^{ \pm}}^{2}\right), \\
& s_{K_{3}}=\frac{c_{\beta}^{2} \mu^{2}}{s_{\beta}^{2} c_{\beta}^{2}}\left\{2\left[\frac{m_{t}^{2}}{\Delta_{\tilde{t}_{i} \tilde{t}_{j}}^{2}}+U_{\times}\right]\left[\mathrm{T}_{1134}\left(m_{\tilde{t}_{i}}^{2}, m_{\tilde{t}_{i}}^{2}, m_{\tilde{b}_{1}}^{2}, m_{H^{ \pm}}^{2}\right)+2 \mathrm{~T}_{1134}\left(m_{\tilde{t}_{i}}^{2}, m_{\tilde{t}_{i}}^{2}, m_{\tilde{t}_{i}}^{2}, m_{H^{ \pm}}^{2}\right)\right]\right. \\
& \left.+\frac{1-4 U_{\times}}{\Delta_{\tilde{t}_{i} \tilde{t}_{j}}}\left[\mathrm{~T}_{134}\left(m_{\tilde{t}_{i}}^{2}, m_{\tilde{b}_{1}}^{2}, m_{H^{ \pm}}^{2}\right)+\mathrm{T}_{134}\left(m_{\tilde{t}_{i}}^{2}, m_{\tilde{t}_{i}}^{2}, m_{H^{ \pm}}^{2}\right)\right]\right\} \\
& s_{L_{1}}=\left\{\frac{2 U_{-}\left[Y_{t}^{2}-3 \eta^{2} m_{t}^{2}\right]\left[\mu^{2}-m_{\tilde{t}_{i}}^{2}\right]}{\Delta_{\tilde{t}_{i} \tilde{t}_{j}}^{2}}+\frac{\eta m_{t}^{2}}{\Delta_{\tilde{t}_{i} \tilde{t}_{j}}}\left[\left(1-3 U_{-}\right) \eta+4 U_{-}\right]-\left[1-U_{-}\right]\left[y_{t}^{2}+1\right]\right\} \mathrm{T}_{134}\left(m_{\tilde{t}_{i}}^{2}, \mu^{2}, 0\right) \\
& -\left\{\left[\mu^{2}-m_{\tilde{t}_{i}}^{2}\right]\left[\frac{\eta m_{t}^{2}}{\Delta_{\tilde{t}_{i} \tilde{t}_{j}}}\left(\eta\left(1-3 U_{-}\right)+4 U_{-}\right)-\left(1-U_{-}\right)\left(y_{t}^{2}+1\right)\right]+[\eta-2]^{2}\left[1-U_{-}\right] m_{t}^{2}\right\} \\
& \times \mathrm{T}_{1134}\left(m_{\tilde{t}_{i}}^{2}, m_{\tilde{t}_{i}}^{2}, \mu^{2}, 0\right)+2\left[\mu^{2}-m_{\tilde{t}_{i}}^{2}+(2 \eta+1) m_{t}^{2}\right] \mathrm{T}_{1134}\left(m_{t}^{2}, m_{t}^{2}, m_{\tilde{t}_{i}}^{2}, \mu^{2}\right) \\
& +\left.c_{\beta}^{2} s_{L_{1}}^{H}\right|_{Y_{t}^{\prime 2} \rightarrow Y_{t}^{2}, y_{t}^{\prime 2} \rightarrow y_{t}^{2}, \eta^{\prime} \rightarrow \eta}+\left.s_{\beta}^{2} s_{L_{1}}^{H}\right|_{m_{H^{ \pm}} \rightarrow 0, Y_{t}^{\prime 2} \rightarrow X_{t}^{2}, y_{t}^{\prime 2} \rightarrow x_{t}^{2}, \eta^{\prime} \rightarrow-2 x_{t}^{2}}, \\
& s_{L_{1}}^{H}=\left\{\frac{4 \eta^{2} m_{t}^{2}}{\Delta_{\tilde{t}_{i} \tilde{t}_{j}}}\left(\frac{3 m_{t}^{2} \eta^{\prime}}{\Delta_{\tilde{t}_{i} \tilde{t}_{j}}}+1\right)-U_{-}\left[2 \frac{\left(m_{t}^{2}-Y_{t}^{\prime 2}\right)\left(3 \eta^{2} m_{t}^{2}-Y_{t}^{2}\right)}{\Delta_{\tilde{t}_{i} \tilde{t}_{j}}^{2}}+\frac{4 \eta m_{t}^{2}}{\Delta_{\tilde{t}_{i} \tilde{t}_{j}}^{2}}+1\right]\right. \\
& \left.-2 \eta^{\prime}\left(\frac{2 m_{t}^{2} y_{t}^{2}}{\Delta_{\tilde{t}_{i} \tilde{t}_{j}}}+1\right)-2 \eta\left(\frac{4 m_{t}^{2} y_{t}^{\prime 2}}{\Delta_{\tilde{t}_{i} \tilde{t}_{j}}}-1\right)-4 y_{t}^{\prime 2}-1\right\} \mathrm{T}_{134}\left(m_{\tilde{t}_{i}}^{2}, m_{\tilde{b}_{1}}^{2}, m_{H^{ \pm}}^{2}\right) \\
& +2\left\{\frac{2 \eta^{2} m_{t}^{2}}{\Delta_{\tilde{t}_{i} \tilde{t}_{j}}}\left(2 U_{\times}\left(4 x_{t}^{2}+3\right)-2 y_{t}^{\prime 2}-1\right)+\frac{2 m_{t}^{2} \eta^{\prime} y_{t}^{2}}{\Delta_{\tilde{t}_{i} \tilde{t}_{j}}}+\left(y_{t}^{2}+1\right) y_{t}^{\prime 2}+1\right. \\
& \left.+\eta\left(\frac{4 m_{t}^{2} y_{t}^{\prime 2}}{\Delta_{\tilde{t}_{i} \tilde{t}_{j}}}-1\right)-x_{t}^{2}\left(4 U_{\times} y_{t}^{2}+1\right)\right\} \mathrm{T}_{134}\left(m_{\tilde{t}_{i}}^{2}, m_{\tilde{t}_{i}}^{2}, m_{H^{ \pm}}^{2}\right) \\
& +\left\{m_{t}^{2}\left\{\eta^{2}\left[\left(1-3 U_{-}\right) y_{t}^{\prime 2}-2\right]+8\left[\frac{\eta^{\prime}\left(y_{t}^{2}+1\right)}{4}+\frac{\eta}{2}-y_{t}^{\prime 2}+\frac{1+U_{-}}{2}\left[\eta\left(y_{t}^{\prime 2}+\frac{1}{2}\right)-\frac{y_{t}^{2}}{4}-\frac{5}{4}\right]\right]\right\}\right. \\
& \left.+\frac{\eta m_{t}^{4}\left[\eta+(3 \eta-4)\left(U_{-}-2 \eta^{\prime}\right)\right]}{\Delta_{\tilde{t}_{i} \tilde{t}_{j}}}-Y_{t}^{\prime 2}\left(1-U_{-}\right)\left(y_{t}^{2}+1\right)\right\} \mathrm{T}_{1134}\left(m_{\tilde{t}_{i}}^{2}, m_{\tilde{t}_{i}}^{2}, m_{\tilde{b}_{1}}^{2}, m_{H^{ \pm}}^{2}\right) \\
& +4 m_{t}^{2}\left\{\eta^{2}\left[\frac{m_{t}^{2}}{\Delta_{\tilde{t}_{i} \tilde{t}_{j}}}-\left(1-5 U_{\times}\right) y_{t}^{\prime 2}-1\right]+\eta^{\prime}\left[\frac{m_{t}^{2} \eta(4-3 \eta)}{\Delta_{\tilde{t}_{i} \tilde{t}_{j}}}+y_{t}^{2}+1\right]+4 \eta\left[\left(1-2 U_{\times}\right) y_{t}^{\prime 2}+1\right]\right. \\
& \left.-y_{t}^{\prime 2}\left[x_{t}^{2}\left(y_{t}^{2}+1\right)+4\right]-y_{t}^{2}-5\right\} \mathrm{T}_{1134}\left(m_{\tilde{t}_{i}}^{2}, m_{\tilde{t}_{i}}^{2}, m_{\tilde{t}_{i}}^{2}, m_{H^{ \pm}}^{2}\right) \text {, }
\end{aligned}
$$




$$
\begin{aligned}
s_{L_{2}}= & \left\{\frac{\eta m_{t}^{2}}{\Delta_{\tilde{t}_{i} \tilde{t}_{j}}}\left[6 U_{-}\left(\frac{\mu^{2}-m_{\tilde{t}_{i}}^{2}}{\Delta_{\tilde{t}_{i} \tilde{t}_{j}}}+\frac{1}{2}\right)-1\right]-\frac{2 U_{-} m_{t}^{2}}{\Delta_{\tilde{t}_{i} \tilde{t}_{j}}}\right\} \mathrm{T}_{134}\left(m_{\tilde{t}_{i}}^{2}, \mu^{2}, 0\right)-2 m_{t}^{2} \mathrm{~T}_{1134}\left(m_{t}^{2}, m_{t}^{2}, m_{\tilde{t}_{i}}^{2}, \mu^{2}\right) \\
& +m_{t}^{2}\left\{\left[\eta\left(1-3 U_{-}\right)+2 U_{-}\right] \frac{\mu^{2}-m_{\tilde{t}_{i}}^{2}}{\Delta_{\tilde{t}_{i} \tilde{t}_{j}}}+(\eta-2)\left(1-U_{-}\right)\right\} \mathrm{T}_{1134}\left(m_{\tilde{t}_{i}}^{2}, m_{\tilde{t}_{i}}^{2}, \mu^{2}, 0\right) \\
& +\left.c_{\beta}^{2} s_{L_{2}}^{H}\right|_{Y_{t}^{\prime 2} \rightarrow Y_{t}^{2}, y_{t}^{\prime 2} \rightarrow y_{t}^{2}, \eta^{\prime} \rightarrow \eta}+\left.s_{\beta}^{2} s_{L_{2}}^{H}\right|_{m_{H^{ \pm}} \rightarrow 0, Y_{t}^{\prime 2} \rightarrow X_{t}^{2}, y_{t}^{\prime 2} \rightarrow x_{t}^{2}, \eta^{\prime} \rightarrow-2 x_{t}^{2}},
\end{aligned}
$$

$$
\begin{aligned}
s_{L_{2}}^{H}= & m_{t}^{2}\left\{\frac{(3 \eta-2)\left[m_{t}^{2}\left(2 \eta^{\prime}-U_{-}\right)+\left(1+U_{-}\right) Y_{t}^{\prime 2}\right]-\eta\left(m_{t}^{2}+4 Y_{t}^{\prime 2}\right)}{\Delta_{\tilde{t}_{i} \tilde{t}_{j}}}-2 \eta-U_{-}-3\right\} \mathrm{T}_{1134}\left(m_{\tilde{t}_{i}}^{2}, m_{\tilde{t}_{i}}^{2}, m_{\tilde{b}_{1}}^{2}, m_{H^{ \pm}}^{2}\right) \\
& +4 m_{t}^{2}\left\{\frac{(3 \eta-2) m_{t}^{2} \eta^{\prime}-\eta\left[m_{t}^{2}-\left(1-5 U_{\times}\right) Y_{t}^{\prime 2}\right]}{\Delta_{\tilde{t}_{i} \tilde{t}_{j}}}+\eta-2 y_{t}^{\prime 2}\left(1-2 U_{\times}\right)-2\right\} \mathrm{T}_{1134}\left(m_{\tilde{t}_{i}}^{2}, m_{\tilde{t}_{i}}^{2}, m_{\tilde{t}_{i}}^{2}, m_{H^{ \pm}}^{2}\right) \\
& +\frac{2 m_{t}^{2}}{\Delta_{\tilde{t}_{i} \tilde{t}_{j}}^{2}}\left\{2 y_{t}^{\prime 2}+U_{-}-\eta\left[\frac{6 m_{t}^{2} \eta^{\prime}}{\Delta_{\tilde{t}_{i} \tilde{t}_{j}}}-3 U_{-} \frac{m_{t}^{2}-Y_{t}^{\prime 2}}{\Delta_{\tilde{t}_{i} \tilde{t}_{j}}}+2\right]\right\} \mathrm{T}_{134}\left(m_{\tilde{t}_{i}}^{2}, m_{\tilde{b}_{1}}^{2}, m_{H^{ \pm}}^{2}\right) \\
& +\frac{4 m_{t}^{2}}{\Delta_{\tilde{t}_{i} \tilde{t}_{j}}}\left\{y_{t}^{\prime 2}-\eta\left[\frac{3 m_{t}^{2} \eta^{\prime}}{\Delta_{\tilde{t}_{i} \tilde{t}_{j}}}+2\left(1-4 U_{\times}\right) y_{t}^{\prime 2}+1\right]\right\} \mathrm{T}_{134}\left(m_{\tilde{t}_{i}}^{2}, m_{\tilde{t}_{i}}^{2}, m_{H^{ \pm}}^{2}\right),
\end{aligned}
$$$$
s_{L_{3}}=2 y_{t}^{2} U_{\times}\left(6 U_{-}\left(\frac{\mu^{2}-m_{\tilde{t}_{i}}^{2}}{\Delta_{\tilde{t}_{i} \tilde{t}_{j}}}+\frac{1}{2}\right)-1\right) \mathrm{T}_{134}\left(m_{\tilde{t}_{i}}^{2}, \mu^{2}, 0\right)
$$$$
+2\left(\left(1-3 U_{-}\right) y_{t}^{2} U_{\times}\left(\mu^{2}-m_{\tilde{t}_{i}}^{2}\right)-\left(1-U_{-}\right)\left(m_{t}^{2}-Y_{t}^{2} U_{\times}\right)\right) \mathrm{T}_{1134}\left(m_{\tilde{t}_{i}}^{2}, m_{\tilde{t}_{i}}^{2}, \mu^{2}, 0\right)
$$$$
+\left.c_{\beta}^{2} s_{L_{3}}^{H}\right|_{Y_{t}^{\prime 2} \rightarrow Y_{t}^{2}, y_{t}^{\prime 2} \rightarrow y_{t}^{2}, \eta^{\prime} \rightarrow \eta}+\left.s_{\beta}^{2} s_{L_{3}}^{H}\right|_{m_{H^{ \pm}} \rightarrow 0, Y_{t}^{\prime 2} \rightarrow X_{t}^{2}, y_{t}^{\prime 2} \rightarrow x_{t}^{2}, \eta^{\prime} \rightarrow-2 x_{t}^{2}},
$$$$
s_{L_{3}}^{H}=-4 y_{t}^{2} U_{\times}\left\{\frac{6 m_{t}^{2} \eta^{\prime}}{\Delta_{\tilde{t}_{i} \tilde{t}_{j}}}-3 U_{-} \frac{m_{t}^{2}-Y_{t}^{\prime 2}}{\Delta_{\tilde{t}_{i} \tilde{t}_{j}}}+2\right\} \mathrm{T}_{134}\left(m_{\tilde{t}_{i}}^{2}, m_{\tilde{b}_{1}}^{2}, m_{H^{ \pm}}^{2}\right)
$$$$
-8 y_{t}^{2} U_{\times}\left\{\frac{3 m_{t}^{2} \eta^{\prime}}{\Delta_{\tilde{t}_{i} \tilde{t}_{j}}}+2\left(1-4 U_{\times}\right) y_{t}^{\prime 2}+1\right\} \mathrm{T}_{134}\left(m_{\tilde{t}_{i}}^{2}, m_{\tilde{t}_{i}}^{2}, m_{H^{ \pm}}^{2}\right)
$$$$
+2 m_{t}^{2}\left\{x_{t}^{2} y_{t}^{2}\left[\frac{6 m_{t}^{2} \eta^{\prime}-\left(1+3 U_{-}\right) m_{t}^{2}-\left(1-3 U_{-}\right) y_{t}^{\prime 2}}{\Delta_{\tilde{t}_{i} \tilde{t}_{j}}}+2\right]-2 y_{t}^{\prime}-U_{-}-1\right\} \mathrm{T}_{1134}\left(m_{\tilde{t}_{i}}^{2}, m_{\tilde{t}_{i}}^{2}, m_{\tilde{b}_{1}}^{2}, m_{H^{ \pm}}^{2}\right)
$$$$
+8 m_{t}^{2}\left\{x_{t}^{2} y_{t}^{2}\left[\frac{3 m_{t}^{2} \eta^{\prime}-m_{t}^{2}+\left(1-5 U_{\times}\right) Y_{t}^{\prime 2}}{\Delta_{\tilde{t}_{i} \tilde{t}_{j}}}+1\right]-y_{t}^{\prime 2}-1\right\} \mathrm{T}_{1134}\left(m_{\tilde{t}_{i}}^{2}, m_{\tilde{t}_{i}}^{2}, m_{\tilde{t}_{i}}^{2}, m_{H^{ \pm}}^{2}\right),
$$

$$
\begin{aligned}
s_{M}= & \frac{1-U_{-}}{\Delta_{\tilde{t}_{i} \tilde{t}_{j}}} \mathrm{~T}_{113}\left(m_{\tilde{b}_{1}}^{2}, m_{\tilde{b}_{1}}^{2}, m_{\tilde{t}_{i}}^{2}\right)+c_{\beta}^{2} s_{M}^{H}+\left.s_{\beta}^{2} s_{M}^{H}\right|_{m_{H^{ \pm}} \rightarrow 0, Y_{t}^{2} \rightarrow X_{t}^{2}, y_{t}^{2} \rightarrow x_{t}^{2}, \eta \rightarrow-2 x_{t}^{2}}, \\
s_{M}^{H}= & \left(\frac{m_{t}^{2}}{\Delta_{\tilde{t}_{i} \tilde{t}_{j}}}\left(1+U_{-}-2 \eta\right)+\left(1-U_{-}\right) y_{t}^{2}\right) \mathrm{T}_{1134}\left(m_{\tilde{b}_{1}}^{2}, m_{\tilde{b}_{1}}^{2}, m_{\tilde{t}_{i}}^{2}, m_{H^{ \pm}}^{2}\right) . \\
s_{N}= & \frac{\Delta_{\tilde{t}_{i} \tilde{t}_{j}}}{2 \Delta_{\tilde{t}_{i} \tilde{b}_{1}}}\left\{\frac{2 \mu^{2} m_{t}^{2}}{c_{\beta}^{2} s_{\beta}^{2} \Delta_{\tilde{t}_{i} \tilde{t}_{j}}^{2}}+y_{t}^{2}\left(U_{-}-1-\frac{2 m_{t}^{2}}{\Delta_{\tilde{t}_{i} \tilde{t}_{j}}}\right)\right\} \mathrm{T}_{134}\left(m_{t}^{2}, m_{\tilde{t}_{i}}^{2}, \mu^{2}\right) \\
& +\left(m_{t}^{2}-m_{\tilde{t}_{i}}^{2}+\mu^{2}\right) \mathrm{T}_{1134}\left(m_{t}^{2}, m_{t}^{2}, m_{\tilde{t}_{i}}^{2}, \mu^{2}\right),
\end{aligned}
$$




$$
\begin{aligned}
& s_{O}=\frac{1}{\Delta_{\tilde{t}_{i} \tilde{b}_{1}}}\left\{y_{t}^{2}\left[-\frac{m_{t}^{2}}{\Delta_{\tilde{t}_{i} \tilde{t}_{j}}}\left(1-U_{-}\left(\frac{m_{t}^{2}}{\Delta_{\tilde{t}_{i} \tilde{t}_{j}}}+1\right)\right)+U_{\times}\left(\frac{4 m_{t}^{2}}{\Delta_{\tilde{t}_{i} \tilde{t}_{j}}}-U_{-}+2\right)+\frac{U_{-}-1}{2}\right]\right. \\
& \left.+\frac{\mu^{2} m_{t}^{2}}{c_{\beta}^{2} s_{\beta}^{2} \Delta_{\tilde{t}_{i} \tilde{t}_{j}}^{2}}\left[1-2 U_{\times}-U_{-}\left(\frac{m_{t}^{2}}{\Delta_{\tilde{t}_{i} \tilde{t}_{j}}}+1\right)\right]\right\} \mathrm{T}_{13}\left(m_{\tilde{t}_{i}}^{2}, \mu^{2}\right) \\
& +\frac{4}{\Delta_{\tilde{t}_{i} \tilde{b}_{1}}}\left\{( 1 - 4 U _ { \times } ) \left[y_{t}^{2}\left(\frac{m_{t}^{2}}{\Delta_{\tilde{t}_{i} \tilde{t}_{j}}}\left(\frac{X_{t}^{2}-m_{t}^{2}}{\Delta_{\tilde{t}_{i} \tilde{t}_{j}}}+U_{-}-1\right)+\frac{U_{-}-1}{2}\right)\right.\right. \\
& \left.\left.+\frac{\mu^{2} m_{t}^{2}}{c_{\beta}^{2} s_{\beta}^{2} \Delta_{\tilde{t}_{i} \tilde{t}_{j}}^{2}}\left(\frac{m_{t}^{2}}{\Delta_{\tilde{t}_{i} \tilde{t}_{j}}}+1-U_{-}\right)\right]-\frac{2 U_{-} U_{\times} \mu^{2} m_{t}^{2}}{c_{\beta}^{2} s_{\beta}^{2} \Delta_{\tilde{t}_{i} \tilde{t}_{j}}^{2}}\right\} \mathrm{T}_{13}\left(m_{\tilde{t}_{i}}^{2}, m_{\tilde{t}_{i}}^{2}\right) \\
& +\frac{4 m_{t}^{4}}{\Delta_{\tilde{t}_{i} \tilde{b}_{1}} \Delta_{\tilde{t}_{j} \tilde{b}_{1}}^{2}}\left\{y_{t}^{2}\left(1-4 U_{\times}\right)\left[\frac{m_{t}^{2}-X_{t}^{2}}{\Delta_{\tilde{t}_{i} \tilde{t}_{j}}}\left(\frac{m_{t}^{2}-X_{t}^{2}}{\Delta_{\tilde{t}_{i} \tilde{t}_{j}}}-2 U_{-}\right)+1-4 U_{\times}\right]\right. \\
& +\frac{\mu^{2}}{c_{\beta}^{2} s_{\beta}^{2} \Delta_{\tilde{t}_{i} \tilde{t}_{j}}}\left[2\left(1-x_{t}^{2} \frac{3 m_{t}^{2}-X_{t}^{2}}{\Delta_{\tilde{t}_{i} \tilde{t}_{j}}}\right)\left(\frac{m_{t}^{2}\left(2 x_{t}^{2}+U_{-}\right)}{\Delta_{\tilde{t}_{i} \tilde{t}_{j}}}-\frac{1}{2}\right)\right. \\
& \left.\left.+\left(1-4 U_{\times}\right) \frac{X_{t}^{4}-m_{t}^{4}}{\Delta_{\tilde{t}_{i} \tilde{t}_{j}}^{2}}+\frac{1}{8}\left(1-16 U_{\times}\right)\right]\right\} \mathrm{T}_{13}\left(m_{\tilde{t}_{i}}^{2}, m_{\tilde{t}_{j}}^{2}\right) \\
& +\frac{m_{t}^{4}}{\Delta_{\tilde{t}_{i} \tilde{b}_{1}} \Delta_{\tilde{t}_{j} \tilde{b}_{1}}^{2}}\left\{\frac{\mu^{2}}{c_{\beta}^{2} s_{\beta}^{2} \Delta_{\tilde{t}_{i} \tilde{t}_{j}}}\left[\frac{m_{t}^{2}}{\Delta_{\tilde{t}_{i} \tilde{t}_{j}}}\left(\left(2 x_{t}^{2}+U_{-}\right)\left(\frac{m_{t}^{2}-X_{t}^{2}}{\Delta_{\tilde{t}_{i} \tilde{t}_{j}}}-U_{-}\right)-1\right)+\frac{7}{2}\left(1-U_{-}\right)\right]\right. \\
& \left.-y_{t}^{2}\left[U_{-}\left(\frac{X_{t}^{2}-m_{t}^{2}}{\Delta_{\tilde{t}_{i} \tilde{t}_{j}}}\right)^{2}+2\left(1-4 U_{\times}\right)\left(\frac{X_{t}^{2}-m_{t}^{2}}{\Delta_{\tilde{t}_{i} \tilde{t}_{j}}}+\frac{U_{-}}{2}\right)\right]\right\} \mathrm{T}_{13}\left(m_{\tilde{t}_{i}}^{2}, m_{\tilde{b}_{1}}^{2}\right) \\
& +\frac{\Delta_{\tilde{t}_{i} \tilde{t}_{j}}}{2 \Delta_{\tilde{t}_{i} \tilde{b}_{1}}}\left\{\frac { m _ { \tilde { t } _ { i } } ^ { 2 } - \mu ^ { 2 } } { \Delta _ { \tilde { t } _ { i } \tilde { t } _ { j } } } \left[y_{t}^{2}\left(1-4 U_{\times}-U_{-}\left(1-\frac{2 m_{t}^{2}}{\Delta_{\tilde{t}_{i} \tilde{t}_{j}}}\left(\frac{X_{t}^{2}-m_{t}^{2}}{\Delta_{\tilde{t}_{i} \tilde{t}_{j}}}+U_{-}-1\right)\right)\right)\right.\right. \\
& \left.+\frac{2 \mu^{2} m_{t}^{2}}{c_{\beta}^{2} s_{\beta}^{2} \Delta_{\tilde{t}_{i} \tilde{t}_{j}}^{2}}\left(U_{-}\left(\frac{m_{t}^{2}}{\Delta_{\tilde{t}_{i} \tilde{t}_{j}}}+1\right)+2 U_{\times}-1\right)\right] \\
& +\left(y_{t}^{2}+1\right)\left[\left(U_{-}-1\right)\left(\frac{m_{t}^{2}}{\Delta_{\tilde{t}_{i} \tilde{t}_{j}}}+1\right)^{2}+4 U_{\times}\left(\frac{m_{t}^{2}}{\Delta_{\tilde{t}_{i} \tilde{t}_{j}}}+1\right)-\left(U_{-}+1\right) U_{\times}\right] \\
& \left.-\frac{\mu^{2} m_{t}^{2}}{c_{\beta}^{2} s_{\beta}^{2} \Delta_{\tilde{t}_{i} \tilde{t}_{j}}^{2}}\left[\left(U_{-}-1\right)\left(\frac{m_{t}^{2}}{\Delta_{\tilde{t}_{i} \tilde{t}_{j}}}+1\right)+2 U_{\times}\right]\right\} \mathrm{T}_{134}\left(m_{\tilde{t}_{i}}^{2}, \mu^{2}, 0\right) \\
& +\frac{\mu^{2}-m_{\tilde{t}_{i}}^{2}}{2}\left\{\frac{\mu^{2} m_{t}^{2}\left(U_{-}-1\right)}{c_{\beta}^{2} s_{\beta}^{2} \Delta_{\tilde{t}_{i} \tilde{t}_{j}}^{2}}-\left(y_{t}^{2}+1\right)\left[\left(U_{-}-1\right)\left(\frac{m_{t}^{2}}{\Delta_{\tilde{t}_{i} \tilde{t}_{j}}}+1\right)+2 U_{\times}\right]\right\} \mathrm{T}_{1134}\left(m_{\tilde{t}_{i}}^{2}, m_{\tilde{t}_{i}}^{2}, \mu^{2}, 0\right) \\
& +\left.c_{\beta}^{2} s_{O}^{H}\right|_{Y_{t}^{\prime 2} \rightarrow Y_{t}^{2}, y_{t}^{\prime 2} \rightarrow y_{t}^{2}, \mu^{\prime} \rightarrow \mu}+\left.s_{\beta}^{2} s_{O}^{H}\right|_{m_{H^{ \pm}} \rightarrow 0, Y_{t}^{\prime 2} \rightarrow X_{t}^{2}, y_{t}^{\prime 2} \rightarrow x_{t}^{2}, \mu^{\prime} \rightarrow 0},
\end{aligned}
$$




$$
\begin{aligned}
& s_{O}^{H}=\left\{m_{t}^{2}\left[1-2 U_{\times}-U_{-}\left(1+\frac{m_{t}^{2}}{\Delta_{\tilde{t}_{i} \tilde{t}_{j}}}\right)\right]\left(y_{t}^{2}-\frac{\mu^{2}}{c_{\beta}^{2} s_{\beta}^{2} \Delta_{\tilde{t}_{i} \tilde{t}_{j}}}\right)\right. \\
& \left.+Y_{t}^{2}\left[2 U_{\times}\left(-\frac{m_{t}^{2}}{\Delta_{\tilde{t}_{i} \tilde{t}_{j}}}+\frac{U_{-}}{2}-1\right)+\frac{1}{2}\left(1-U_{-}\right)\right]\right\} \mathrm{T}_{13}\left(m_{\tilde{t}_{i}}^{2}, m_{H^{ \pm}}^{2}\right) \\
& -m_{t}^{2}\left\{\frac { 2 m _ { t } ^ { 2 } } { \Delta _ { \tilde { t } _ { i } \tilde { t } _ { j } } } \left[\frac{\mu^{2}}{c_{\beta}^{2} s_{\beta}^{2} \Delta_{\tilde{t}_{i} \tilde{t}_{j}}}\left(\frac{\mu^{\prime 2}}{c_{\beta}^{2} s_{\beta}^{2} \Delta_{\tilde{t}_{i} \tilde{t}_{j}}}-\left(x_{t}^{2}+1\right)^{2}\right)+\frac{\mu^{\prime 2}}{c_{\beta}^{2} s_{\beta}^{2} \Delta_{\tilde{t}_{i} \tilde{t}_{j}}}\left(x_{t}^{4}+x_{t}^{2}-1-\left(x_{t}^{2}+2\right) y_{t}^{2}\right)\right.\right. \\
& \left.+\left(x_{t}^{2}+1\right)\left(y_{t}^{2}+1\right)\left(y_{t}^{\prime 2}+1\right)\right] \\
& \left.+\left(U_{-}-1\right)\left(y_{t}^{2}+1\right)\left[\frac{\mu^{\prime 2}}{c_{\beta}^{2} s_{\beta}^{2} \Delta_{\tilde{t}_{i} \tilde{t}_{j}}}-\left(x_{t}^{2}+1\right)\left(y_{t}^{\prime 2}+1\right)\right]\right\} \mathrm{T}_{1134}\left(m_{\tilde{t}_{i}}^{2}, m_{\tilde{t}_{i}}^{2}, m_{\tilde{t}_{i}}^{2}, m_{H^{ \pm}}^{2}\right) \\
& +\frac{\Delta_{\tilde{t}_{i} \tilde{t}_{j}}}{\Delta_{\tilde{t}_{i} \tilde{b}_{1}}}\left\{\frac { U _ { - } - 1 } { 2 } \left[\frac{\mu^{\prime 2}-\mu^{2}}{c_{\beta}^{2} s_{\beta}^{2} \Delta_{\tilde{t}_{i} \tilde{t}_{j}}}+y_{t}^{2}+y_{t}^{\prime 2}\left(\frac{2 m_{t}^{2}}{\Delta_{\tilde{t}_{i} \tilde{t}_{j}}}\left(1-2 y_{t}^{2}\left(\frac{m_{t}^{2}}{\Delta_{\tilde{t}_{i} \tilde{t}_{j}}}+x_{t}^{2}+2 U_{\times}\right)\right)+y_{t}^{2}+1\right)\right.\right. \\
& +\frac{2 m_{t}^{2}}{\Delta_{\tilde{t}_{i} \tilde{t}_{j}}}\left(\frac{\mu^{\prime 2}}{c_{\beta}^{2} s_{\beta}^{2} \Delta_{\tilde{t}_{i} \tilde{t}_{j}}}\left(\left(-\frac{m_{t}^{2}}{\Delta_{\tilde{t}_{i} \tilde{t}_{j}}}+1-2 U_{\times}\right) x_{t}^{2}+\frac{m_{t}^{2}\left(2 x_{t}^{2}+3\right) y_{t}^{2}}{\Delta_{\tilde{t}_{i} \tilde{t}_{j}}}+1\right)\right. \\
& -\frac{\mu^{2}}{c_{\beta}^{2} s_{\beta}^{2} \Delta_{\tilde{t}_{i} \tilde{t}_{j}}}\left(\frac{m_{t}^{2}}{\Delta_{\tilde{t}_{i} \tilde{t}_{j}}}\left(\frac{\mu^{\prime 2}}{c_{\beta}^{2} s_{\beta}^{2} \Delta_{\tilde{t}_{i} \tilde{t}_{j}}}-2 x_{t}^{2}\left(x_{t}^{2}+1\right)\right)+x_{t}^{2}+2\right) \\
& \left.\left.+y_{t}^{2}\left(1-x_{t}^{2}\left(\frac{2 m_{t}^{2}}{\Delta_{\tilde{t}_{i} \tilde{t}_{j}}}+1\right)\right)+1\right)+1\right] \\
& +\frac{m_{t}^{2}}{\Delta_{\tilde{t}_{i} \tilde{t}_{j}}^{2}}\left[\frac { 2 m _ { t } ^ { 4 } } { \Delta _ { \tilde { t } _ { i } \tilde { t } _ { j } } ^ { 2 } } \left(\frac{\mu^{\prime 2}\left(x_{t}^{2}\left(1+2 x_{t}^{2}\right)-2 y_{t}^{2}\left(1+x_{t}^{2}\right)\right)}{c_{\beta}^{2} s_{\beta}^{2} \Delta_{\tilde{t}_{i} \tilde{t}_{j}}}+\frac{\mu^{2}}{c_{\beta}^{2} s_{\beta}^{2} \Delta_{\tilde{t}_{i} \tilde{t}_{j}}}\left(\frac{\mu^{\prime 2}}{c_{\beta}^{2} s_{\beta}^{2} \Delta_{\tilde{t}_{i} \tilde{t}_{j}}}-2 x_{t}^{2}\left(x_{t}^{2}+1\right)\right)\right.\right. \\
& \left.+y_{t}^{2}\left(2 x_{t}^{2} y_{t}^{\prime 2}+y_{t}^{\prime 2}+x_{t}^{2}\right)\right) \\
& +\frac{m_{t}^{2}}{\Delta_{\tilde{t}_{i} \tilde{t}_{j}}}\left(\frac{\mu^{\prime 2}\left(2 x_{t}^{4}-1\right)}{c_{\beta}^{2} s_{\beta}^{2} \Delta_{\tilde{t}_{i} \tilde{t}_{j}}}+\frac{\mu^{2}}{c_{\beta}^{2} s_{\beta}^{2} \Delta_{\tilde{t}_{i} \tilde{t}_{j}}}\left(\frac{\mu^{\prime 2}}{c_{\beta}^{2} s_{\beta}^{2} \Delta_{\tilde{t}_{i} \tilde{t}_{j}}}-2 x_{t}^{2}\left(x_{t}^{2}+1\right)+1\right)\right. \\
& \left.-y_{t}^{\prime 2}-y_{t}^{2}\left(y_{t}^{\prime 2}+2 x_{t}^{2}\left(2 x_{t}^{2} y_{t}^{\prime 2}+y_{t}^{\prime 2}+x_{t}^{2}\right)+1\right)-1\right) \\
& \left.\left.+x_{t}^{2}\left(\frac{\mu^{\prime 2}-\mu^{2}}{c_{\beta}^{2} s_{\beta}^{2} \Delta_{\tilde{t}_{i} \tilde{t}_{j}}}+y_{t}^{\prime 2}+y_{t}^{\prime 2} y_{t}^{2}+y_{t}^{2}+1\right)\right]\right\} \mathrm{T}_{134}\left(m_{\tilde{t}_{i}}^{2}, m_{\tilde{t}_{i}}^{2}, m_{H^{ \pm}}^{2}\right) \text {. }
\end{aligned}
$$




$$
\begin{aligned}
& +\frac{m_{t}^{4} \Delta_{\tilde{t}_{i} \tilde{t}_{j}}}{\Delta_{\tilde{t}_{i} \tilde{b}_{1}} \Delta_{\tilde{t}_{j} \tilde{b}_{1}}^{2}}\left\{\frac{\mu^{2}\left(1-U_{-} y_{t}^{\prime 2}\right)}{c_{\beta}^{2} s_{\beta}^{2} \Delta_{\tilde{t}_{i} \tilde{t}_{j}}}-x_{t}^{4}\left[\left(U_{-} y_{t}^{2}+1\right) y_{t}^{\prime 2}+\frac{U_{-}+1}{2}+\left(1-2 U_{\times}\right)\left(\frac{\mu^{\prime 2}-\mu^{2}}{c_{\beta}^{2} s_{\beta}^{2} \Delta_{\tilde{t}_{i} \tilde{t}_{j}}}+y_{t}^{2}\right)\right]\right. \\
& +\frac{\mu^{\prime 2} \mu^{2}}{c_{\beta}^{4} s_{\beta}^{4} \Delta_{\tilde{t}_{i} \tilde{t}_{j}}^{2}}\left[\frac{U_{-}-1}{2}+U_{-} U_{\times}\right]-\left(1-6 U_{\times}\right)\left[\frac{\mu^{\prime 2}\left(1-2 U_{\times}\right)+\mu^{2}}{c_{\beta}^{2} s_{\beta}^{2} \Delta_{\tilde{t}_{i}} \tilde{t}_{j}}+y_{t}^{\prime 2}+\frac{U_{-}+1}{2}\right] \\
& +y_{t}^{2}\left[\frac{\mu^{\prime 2}\left(1-8 U_{-} U_{\times}\right)}{c_{\beta}^{2} s_{\beta}^{2} \Delta_{\tilde{t}_{i} \tilde{t}_{j}}}-U_{-}\left(1-10 U_{\times}\right) y_{t}^{\prime 2}-2 U_{\times}\right] \\
& -x_{t}^{2}\left[\frac{\mu^{\prime 2}\left(4 U_{\times} y_{t}^{2}+U_{-} U_{\times}+1\right)}{c_{\beta}^{2} s_{\beta}^{2} \Delta_{\tilde{t}_{i} \tilde{t}_{j}}}-\frac{2 \mu^{2}}{c_{\beta}^{2} s_{\beta}^{2} \Delta_{\tilde{t}_{i} \tilde{t}_{j}}}-4 U_{\times}+2 y_{t}^{\prime 2}\left(y_{t}^{2}\left(1-5 U_{\times}\right)+U_{-}\right)\right. \\
& \left.+\frac{U_{-}+1}{2}\left(\frac{\mu^{2}}{c_{\beta}^{2} s_{\beta}^{2} \Delta_{\tilde{t}_{i} \tilde{t}_{j}}}+2\right)+U_{-}\left(2-5 U_{\times}\right)\left(\frac{\mu^{2}-\mu^{2}}{c_{\beta}^{2} s_{\beta}^{2} \Delta_{\tilde{t}_{i} \tilde{t}_{j}}}+y_{t}^{2}\right)\right] \\
& +\frac{m_{t}^{2}}{\Delta_{\tilde{t}_{i} \tilde{t}_{j}}}\left[-\frac{\mu^{\prime 2} 4\left(1-6 U_{\times}\right) y_{t}^{2}}{c_{\beta}^{2} s_{\beta}^{2} \Delta_{\tilde{t}_{i} \tilde{t}_{j}}}-\frac{\mu^{2}}{2 c_{\beta}^{2} s_{\beta}^{2} \Delta_{\tilde{t}_{i} \tilde{t}_{j}}}\left(\frac{\mu^{\prime 2} 12 U_{\times}}{c_{\beta}^{2} s_{\beta}^{2} \Delta_{\tilde{t}_{i} \tilde{t}_{j}}}+U_{-}\left(3-20 U_{\times}\right)+1\right)\right. \\
& +U_{-}\left(-\frac{4 U_{\times} \mu^{\prime 2}}{c_{\beta}^{2} s_{\beta}^{2} \Delta_{\tilde{t}_{i} \tilde{t}_{j}}}+\frac{2 \mu^{\prime 2}}{c_{\beta}^{2} s_{\beta}^{2} \Delta_{\tilde{t}_{i} \tilde{t}_{j}}}+2 y_{t}^{\prime 2}-10 U_{\times} y_{t}^{2}+3 y_{t}^{2}+1\right) \\
& \left.+4\left(1-5 U_{\times}\right) y_{t}^{\prime 2} y_{t}^{2}-4 U_{\times}+1\right] \\
& +\frac{m_{t}^{4}}{\Delta_{\tilde{t}_{i} \tilde{t}_{j}}^{2}}\left[\frac{\mu^{\prime 2}\left(8 U_{-} y_{t}^{2}+2 U_{\times}-1\right)}{c_{\beta}^{2} s_{\beta}^{2} \Delta_{\tilde{t}_{i} \tilde{t}_{j}}}+\frac{\mu^{2}}{c_{\beta}^{2} s_{\beta}^{2} \Delta_{\tilde{t}_{i} \tilde{t}_{j}}}\left(-\frac{3 U_{-} \mu^{\prime 2}}{c_{\beta}^{2} s_{\beta}^{2} \Delta_{\tilde{t}_{i} \tilde{t}_{j}}}+3-10 U_{\times}\right)\right. \\
& \left.-\left(3-10 U_{\times}\right) y_{t}^{2}-\frac{U_{-}}{2}-y_{t}^{\prime 2}\left(5 U_{-} y_{t}^{2}+1\right)-\frac{1}{2}\right] \\
& +\frac{m_{t}^{6}}{\Delta_{\tilde{t}_{i} \tilde{t}_{j}}^{3}}\left[\frac{2 \mu^{\prime 2}}{c_{\beta}^{2} s_{\beta}^{2} \Delta_{\tilde{t}_{i} \tilde{t}_{j}}}\left(\frac{\mu^{2}}{c_{\beta}^{2} s_{\beta}^{2} \Delta_{\tilde{t}_{i} \tilde{t}_{j}}}-2 y_{t}^{2}\right)-\frac{U_{-} \mu^{2}}{c_{\beta}^{2} s_{\beta}^{2} \Delta_{\tilde{t}_{i} \tilde{t}_{j}}}+y_{t}^{2}\left(2 y_{t}^{\prime 2}+U_{-}\right)\right] \\
& \left.+\left(1-10 U_{\times}\right)\left(1-2 U_{\times}\right)\left(\frac{\mu^{2}}{c_{\beta}^{2} s_{\beta}^{2} \Delta_{\tilde{t}_{i} \tilde{t}_{j}}}-y_{t}^{2}\right)\right\} \mathrm{T}_{134}\left(m_{\tilde{t}_{i}}^{2}, m_{\tilde{b}_{1}}^{2}, m_{H^{ \pm}}^{2}\right) \\
& +\frac{m_{t}^{4} \Delta_{\tilde{t}_{i} \tilde{t}_{j}}}{\Delta_{\tilde{t}_{i} \tilde{b}_{1}} \Delta_{\tilde{t}_{j} \tilde{b}_{1}}^{2}}\left\{\frac{\mu^{\prime 2}}{c_{\beta}^{2} s_{\beta}^{2} \Delta_{\tilde{t}_{i} \tilde{t}_{j}}}\left(x_{t}^{2}-y_{t}^{2}\right)\left[\frac{m_{t}^{4}\left(12 x_{t}^{4}+1-4 U_{\times}\right)}{\Delta_{\tilde{t}_{i} \tilde{t}_{j}}^{2}}+2 U_{-} U_{\times}\left(\frac{3 m_{t}^{2}}{\Delta_{\tilde{t}_{i} \tilde{t}_{j}}}-x_{t}^{2}\right)+1-5 U_{\times}\right]\right. \\
& +\frac{\mu^{2}}{2 c_{\beta}^{2} s_{\beta}^{2} \Delta_{\tilde{t}_{i} \tilde{t}_{j}}}\left[\frac{\mu^{\prime 2}}{c_{\beta}^{2} s_{\beta}^{2} \Delta_{\tilde{t}_{i} \tilde{t}_{j}}}+\frac{m_{t}^{2}\left(4 U_{-} y_{t}^{\prime 2}+8 U_{\times}^{2}-1\right)}{\Delta_{\tilde{t}_{i} \tilde{t}_{j}}}-3 x_{t}^{2}\right. \\
& \left.-2 U_{\times}\left(-2 x_{t}^{2}\left(U_{-} x_{t}^{2}-6 U_{\times}+2\right)+U_{-}\left(1+6 U_{\times}\right)\right)\right] \\
& \left.+\left(1-4 U_{\times}\right) y_{t}^{\prime 2} y_{t}^{2}\left[\frac{m_{t}^{4}}{\Delta_{\tilde{t}_{i} \tilde{j}_{j}}^{2}}+2 U_{-}\left(x_{t}^{2}-\frac{m_{t}^{2}}{\Delta_{\tilde{t}_{i} \tilde{t}_{j}}}\right)+x_{t}^{4}+1-6 U_{\times}\right]\right\} \\
& \times \mathrm{T}_{134}\left(m_{\tilde{t}_{i}}^{2}, m_{\tilde{t}_{j}}^{2}, m_{H^{ \pm}}^{2}\right) \\
& +\Delta_{\tilde{t}_{i} \tilde{t}_{j}}\left\{\frac { m _ { t } ^ { 4 } } { \Delta _ { \tilde { t } _ { i } \tilde { t } _ { j } } ^ { 2 } } \left[\frac{\mu^{2}}{c_{\beta}^{2} s_{\beta}^{2} \Delta_{\tilde{t}_{i} \tilde{t}_{j}}}\left(2 x_{t}^{2}+1-\frac{\mu^{\prime 2}}{c_{\beta}^{2} s_{\beta}^{2} \Delta_{\tilde{t}_{i} \tilde{t}_{j}}}\right)+\frac{\mu^{\prime 2}}{c_{\beta}^{2} s_{\beta}^{2} \Delta_{\tilde{t}_{i} \tilde{t}_{j}}}\left(2 y_{t}^{2}-x_{t}^{2}+1\right)\right.\right. \\
& \left.-\left(y_{t}^{2}+1\right)\left(y_{t}^{\prime 2}+2 x_{t}^{2}+1\right)-\frac{U_{-}-1}{2}\left(y_{t}^{2}+1-\frac{\mu^{2}}{c_{\beta}^{2} s_{\beta}^{2} \Delta_{\tilde{t}_{i} \tilde{t}_{j}}}\right)\right] \\
& +\frac{U_{-}-1}{2}\left[U_{\times}\left(\frac{\mu^{\prime 2}-\mu^{2}}{c_{\beta}^{2} s_{\beta}^{2} \Delta_{\tilde{t}_{i} \tilde{t}_{j}}}+y_{t}^{2}+1\right)-\frac{m_{t}^{2}}{\Delta_{\tilde{t}_{i} \tilde{t}_{j}}}\left(\frac{\mu^{\prime 2}\left(1+2 y_{t}^{2}\right)}{c_{\beta}^{2} s_{\beta}^{2} \Delta_{\tilde{t}_{i} \tilde{t}_{j}}}-2\left(y_{t}^{2}+1\right) y_{t}^{\prime 2}\right)\right] \\
& \left.+\left(y_{t}^{2}+1\right) y_{t}^{\prime 2}\left(\frac{U_{-}-1}{2}+U_{\times}\right)\right\} \mathrm{T}_{1134}\left(m_{\tilde{t}_{i}}^{2}, m_{\tilde{t}_{i}}^{2}, m_{\tilde{b}_{1}}^{2}, m_{H^{ \pm}}^{2}\right)
\end{aligned}
$$




\section{D.3 Genuine two-loop tadpoles}

The explicit expressions for the genuine two-loop tadpoles of the Higgs bosons are given by

$$
\begin{aligned}
& T_{h}^{(2)}=\frac{N_{c} s_{\beta} h_{t}^{3} m_{t}}{128 \sqrt{2} \pi^{4}}\left\{t_{A}+\sum_{\substack{i=1 \\
j \neq i}}^{2}\left[-x_{t}^{2} t_{B}+\left(1+x_{t}^{2}\right) t_{C}-x_{t}^{2} t_{D_{1}}-t_{D_{2}}+t_{D_{3}}\right]\right\}, \\
& T_{H}^{(2)}=-\frac{N_{c} c_{\beta} h_{t}^{3} m_{t}}{128 \sqrt{2} \pi^{4}}\left\{t_{A}+\sum_{\substack{i=1 \\
j \neq i}}^{2}\left[\frac{\eta}{2} t_{B}+\left(1-\frac{\eta}{2}\right) t_{C}+\frac{\eta}{2} t_{D_{1}}-t_{D_{2}}\right]\right\}, \\
& T_{A}^{(2)}=\sum_{\substack{i=1 \\
j \neq i}}^{2} \frac{N_{c} h_{t}^{3} m_{t}}{128 \sqrt{2} \pi^{4} s_{\beta}} \frac{\Im \mathfrak{m}\left[X_{t} \mu^{*}\right]}{s_{\beta} c_{\beta} \Delta_{\tilde{t}_{i} \tilde{t}_{j}}}\left\{t_{B}-t_{C}+t_{D_{1}}\right\} . \\
& t_{A}=s_{A}+\sum_{i=1}^{2}\left(m_{t}^{2}-m_{\tilde{t}_{i}}^{2}+\mu^{2}\right) \mathrm{T}_{1134}\left(m_{t}^{2}, m_{t}^{2}, m_{\tilde{t}_{i}}^{2}, \mu^{2}\right), \\
& t_{B}=s_{D}+s_{G}+s_{H}, \\
& t_{C}=s_{I_{1}}+s_{I_{2}}+\frac{1}{2}\left(1-U_{-}\right)\left(\mu^{2}-m_{\tilde{t}_{i}}^{2}\right) \mathrm{T}_{1134}\left(m_{\tilde{t}_{i}}^{2}, m_{\tilde{t}_{i}}^{2}, \mu^{2}, 0\right) \\
& +c_{\beta}^{2} t_{C}^{H}+\left.s_{\beta}^{2} t_{C}^{H}\right|_{m_{H^{ \pm}} \rightarrow 0, Y_{t}^{2} \rightarrow X_{t}^{2}, y_{t}^{2} \rightarrow x_{t}^{2}, \eta \rightarrow-2 x_{t}^{2}}, \\
& t_{C}^{H}=\left\{m_{t}^{2}\left(\eta-\frac{1+U_{-}}{2}\right)-\frac{1-U_{-}}{2} Y_{t}^{2}\right\} \mathrm{T}_{1134}\left(m_{\tilde{t}_{i}}^{2}, m_{\tilde{t}_{i}}^{2}, m_{\tilde{b}_{1}}^{2}, m_{H^{ \pm}}^{2}\right) \\
& +2 m_{t}^{2}\left(\eta-x_{t}^{2} y_{t}^{2}-1\right) \mathrm{T}_{1134}\left(m_{\tilde{t}_{i}}^{2}, m_{\tilde{t}_{i}}^{2}, m_{\tilde{t}_{i}}^{2}, m_{H^{ \pm}}^{2}\right), \\
& t_{D_{1}}=\left\{\frac{1}{2}-U_{-}\left(\frac{\mu^{2}-m_{\tilde{t}_{i}}^{2}}{\Delta_{\tilde{t}_{i} \tilde{t}_{j}}}+\frac{1}{2}\right)\right\} \mathrm{T}_{134}\left(m_{\tilde{t}_{i}}^{2}, 0, \mu^{2}\right) \\
& +c_{\beta}^{2} t_{D_{1}}^{H}+\left.s_{\beta}^{2} t_{D_{1}}^{H}\right|_{m_{H^{ \pm}} \rightarrow 0, Y_{t}^{2} \rightarrow X_{t}^{2}, y_{t}^{2} \rightarrow x_{t}^{2}, \eta \rightarrow-2 x_{t}^{2}}, \\
& t_{D_{1}}^{H}=\left\{\frac{2 \eta m_{t}^{2}+U_{-}\left(Y_{t}^{2}-m_{t}^{2}\right)}{\Delta_{\tilde{t}_{i} \tilde{t}_{j}}}+1\right\} \mathrm{T}_{134}\left(m_{\tilde{t}_{i}}^{2}, m_{\tilde{b}_{1}}^{2}, m_{H^{ \pm}}^{2}\right) \\
& +\left\{\frac{2 \eta m_{t}^{2}+Y_{t}^{2}\left(1-4 U_{\times}\right)}{\Delta_{\tilde{t}_{i} \tilde{t}_{j}}}+1\right\} \mathrm{T}_{134}\left(m_{\tilde{t}_{i}}^{2}, m_{\tilde{t}_{i}}^{2}, m_{H^{ \pm}}^{2}\right), \\
& t_{D_{2}}=\frac{1-U_{-}}{2} \mathrm{~T}_{134}\left(m_{\tilde{t}_{i}}^{2}, \mu^{2}, 0\right)+c_{\beta}^{2} t_{D_{2}}^{H}+\left.s_{\beta}^{2} t_{D_{2}}^{H}\right|_{m_{H^{ \pm}} \rightarrow 0, Y_{t}^{2} \rightarrow X_{t}^{2}, y_{t}^{2} \rightarrow x_{t}^{2}, \eta \rightarrow-2 x_{t}^{2}}, \\
& t_{D_{2}}^{H}=\frac{2 y_{t}^{2}+1+U_{-}}{2} \mathrm{~T}_{134}\left(m_{\tilde{t}_{i}}^{2}, m_{\tilde{b}_{1}}^{2}, m_{H^{ \pm}}^{2}\right)+\left(y_{t}^{2}+1\right) \mathrm{T}_{134}\left(m_{\tilde{t}_{i}}^{2}, m_{\tilde{t}_{i}}^{2}, m_{H^{ \pm}}^{2}\right), \\
& t_{D_{3}}=c_{\beta}^{2} \frac{\mu^{2}}{s_{\beta}^{2} c_{\beta}^{2} \Delta_{\tilde{t}_{i} \tilde{t}_{j}}}\left[\mathrm{~T}_{134}\left(m_{\tilde{t}_{i}}^{2}, m_{\tilde{b}_{1}}^{2}, m_{H^{ \pm}}^{2}\right)+\mathrm{T}_{134}\left(m_{\tilde{t}_{i}}^{2}, m_{\tilde{t}_{i}}^{2}, m_{H^{ \pm}}^{2}\right)\right] .
\end{aligned}
$$

\section{D.4 One-loop self-energies with counterterm insertions}

The one-loop self-energies with counterterm insertion are part of the full two-loop selfenergies. They are given in the following:

$$
\begin{aligned}
& \Sigma_{h h}^{(2) \mathrm{ct}}=\frac{N_{c} s_{\beta}^{2} h_{t}^{2}}{16 \pi^{2}}\left\{s_{A_{1}}^{\mathrm{ct}}+s_{A_{2}}^{\mathrm{ct}}\right\} \\
& +\sum_{\substack{i=1 \\
j \neq i}}^{2} \frac{N_{c} s_{\beta}^{2} h_{t}^{2}}{16 \pi^{2}}\left\{\left(1+x_{t}^{2}\right) s_{A_{3}}^{\mathrm{ct}}+\left(1+x_{t}^{2}\right)^{2} s_{A_{4}}^{\mathrm{ct}}+x_{t}^{2}\left(1-4 U_{\times}\right) s_{A_{5}}^{\mathrm{ct}}-2 x_{t}^{4} s_{B_{1}}^{\mathrm{ct}}\right. \\
& \left.+x_{t}^{2} s_{B_{2}}^{\mathrm{ct}}+x_{t}^{2}\left(1-4 U_{\times}\right) s_{D_{1}}^{\mathrm{ct}}+x_{t}^{2}\left(1+x_{t}^{2}\right) s_{D_{2}}^{\mathrm{ct}}\right\},
\end{aligned}
$$




$$
\begin{aligned}
& \Sigma_{h H}^{(2) \mathrm{ct}}=-\frac{N_{c} s_{\beta} c_{\beta} h_{t}^{2}}{16 \pi^{2}}\left\{s_{A_{1}}^{\mathrm{ct}}+s_{A_{2}}^{\mathrm{ct}}\right\} \\
& -\sum_{\substack{i=1 \\
j \neq i}}^{2} \frac{N_{c} s_{\beta} c_{\beta} h_{t}^{2}}{16 \pi^{2}}\left\{\left(1-\frac{\eta}{4}+\frac{x_{t}^{2}}{2}\right) s_{A_{3}}^{\mathrm{ct}}-\left(\frac{\eta}{2}-1\right)\left(1+x_{t}^{2}\right) s_{A_{4}}^{\mathrm{ct}}-\frac{\eta}{2}\left(1-4 U_{\times}\right) s_{A_{5}}^{\mathrm{ct}}\right. \\
& +\eta x_{t}^{2} s_{B_{1}}^{\mathrm{ct}}+\left(\frac{x_{t}^{2}}{2}-\frac{\eta}{4}\right) s_{B_{2}}^{\mathrm{ct}}+\frac{\Im \mathfrak{m}\left[X_{t} \mu^{*}\right]}{s_{\beta} c_{\beta} \Delta_{\tilde{t}_{i} \tilde{t}_{j}}}\left(x_{t}^{2} s_{B_{3}}^{\mathrm{ct}}-s_{B_{4}}^{\mathrm{ct}}\right) \\
& -\left(\frac{\eta}{2}+x_{t}^{2}\right)\left[\left(1-4 U_{\times}\right) s_{C_{1}}^{\mathrm{ct}}+\left(1+x_{t}^{2}\right) s_{C_{2}}^{\mathrm{ct}}\right]+x_{t}^{2} s_{D_{2}}^{\mathrm{ct}} \\
& \left.+\left(\frac{x_{t}^{2}}{2}-\frac{\eta}{4}\right)\left[\left(1-4 U_{\times}\right) s_{D_{1}}^{\mathrm{ct}}+x_{t}^{2} s_{D_{2}}^{\mathrm{ct}}\right]+\left(\frac{\eta^{2}}{4}-x_{t}^{2} y_{t}^{2}\right) s_{D_{3}}^{\mathrm{ct}}\right\}, \\
& \Sigma_{H H}^{(2) \mathrm{ct}}=\frac{N_{c} c_{\beta}^{2} h_{t}^{2}}{16 \pi^{2}}\left\{s_{A_{1}}^{\mathrm{ct}}+s_{A_{2}}^{\mathrm{ct}}\right\} \\
& +\sum_{\substack{i=1 \\
j \neq i}}^{2} \frac{N_{c} c_{\beta}^{2} h_{t}^{2}}{16 \pi^{2}}\left\{-\left(\frac{\eta}{2}-1\right) s_{A_{3}}^{\mathrm{ct}}+\left(\frac{\eta}{2}-1\right)^{2} s_{A_{4}}^{\mathrm{ct}}-\frac{m_{t}^{2} \eta^{2}-Y_{t}^{2}}{\Delta_{\tilde{t}_{i} \tilde{t}_{j}}} s_{A_{5}}^{\mathrm{ct}}\right. \\
& -\frac{\eta^{2}}{2} s_{B_{1}}^{\mathrm{ct}}-\frac{\eta}{2} s_{B_{2}}^{\mathrm{ct}}-\frac{\Im \mathfrak{m}\left[X_{t} \mu^{*}\right]}{s_{\beta} c_{\beta} \Delta_{\tilde{t}_{i} \tilde{t}_{j}}}\left(\eta s_{B_{3}}^{\mathrm{ct}}+2 s_{B_{4}}^{\mathrm{ct}}\right) \\
& +\left[\eta\left(1-4 U_{\times}\right)-2 \frac{m_{t}^{2} \eta^{2}-Y_{t}^{2}}{\Delta_{\tilde{t}_{i} \tilde{t}_{j}}}\right] s_{C_{1}}^{\mathrm{ct}}+\left(\frac{\eta}{2}-1\right)\left(\eta+2 x_{t}^{2}\right) s_{C_{2}}^{\mathrm{ct}} \\
& \left.+\frac{\eta}{2}\left(1-4 U_{\times}\right) s_{D_{1}}^{\mathrm{ct}}-\left(\frac{\eta}{2}-1\right) x_{t}^{2} s_{D_{2}}^{\mathrm{ct}}+\left(\frac{\eta^{2}}{2}-2 x_{t}^{2} y_{t}^{2}\right) s_{D_{3}}^{\mathrm{ct}}\right\}, \\
& +\sum_{\substack{i=1 \\
j \neq i}}^{2} \frac{N_{c} c_{\beta}^{2} h_{t}^{2}}{16 \pi^{2}}\left\{-\left(\frac{\eta^{2}}{4}-x_{t}^{2} y_{t}^{2}\right) s_{A_{4}}^{\mathrm{ct}}+\frac{m_{t}^{2} \eta^{2}+\left(1-4 U_{\times}\right) Y_{t}^{2}}{\Delta_{\tilde{t}_{i} \tilde{t}_{j}}} s_{A_{5}}^{\mathrm{ct}}+\left(\frac{\eta^{2}}{2}-2 x_{t}^{2} y_{t}^{2}\right) s_{B_{1}}^{\mathrm{ct}}\right. \\
& +\frac{\Im \mathfrak{m}\left[X_{t} \mu^{*}\right]}{s_{\beta} c_{\beta} \Delta_{\tilde{t}_{i} \tilde{t}_{j}}} \eta s_{B_{3}}^{\mathrm{ct}}+2\left(\frac{\eta}{2}+\frac{m_{t}^{2} \eta^{2}+\left(1-4 U_{\times}\right) Y_{t}^{2}}{\Delta_{\tilde{t}_{i} \tilde{t}_{j}}}\right) s_{C_{1}}^{\mathrm{ct}}-\left(\frac{\eta^{2}}{2}-2 x_{t}^{2} y_{t}^{2}\right) s_{C_{2}}^{\mathrm{ct}} \\
& \left.-\frac{\eta}{2} s_{D_{1}}^{\mathrm{ct}}+\left(\frac{\eta^{2}}{2}-2 x_{t}^{2} y_{t}^{2}\right)\left[\left(1-4 U_{\times}\right) s_{D_{3}}^{\mathrm{ct}}+x_{t}^{2} s_{D_{4}}^{\mathrm{ct}}\right]\right\},
\end{aligned}
$$

$\Sigma_{h A}^{(2) \mathrm{ct}}=\sum_{\substack{i=1 \\ j \neq i}}^{2} \frac{N_{c} s_{\beta} c_{\beta} h_{t}^{2}}{16 \pi^{2}} \frac{\Im \mathfrak{m}\left[X_{t} \mu^{*}\right]}{s_{\beta} c_{\beta} \Delta_{\tilde{t}_{i} \tilde{t}_{j}}}\left\{-\frac{1}{2} s_{A_{3}}^{\mathrm{ct}}-\left(1+x_{t}^{2}\right) s_{A_{4}}^{\mathrm{ct}}-\left(1-4 U_{\times}\right) s_{A_{5}}^{\mathrm{ct}}\right.$

$$
\begin{gathered}
\qquad x_{t}^{2} s_{B_{1}}^{\mathrm{ct}}-\frac{1}{2} s_{B_{2}}^{\mathrm{ct}}-\frac{s_{\beta} c_{\beta} \Delta_{\tilde{t}_{i} \tilde{j}_{j}}}{\Im \mathfrak{m}\left[X_{t} \mu^{*}\right]} \frac{\eta}{2}\left(x_{t}^{2} s_{B_{3}}^{\mathrm{ct}}-s_{B_{4}}^{\mathrm{ct}}\right) \\
-\left(1-4 U_{\times}\right)\left(s_{C_{1}}^{\mathrm{ct}}+\frac{1}{2} s_{D_{1}}^{\mathrm{ct}}\right)-\left(1+x_{t}^{2}\right)\left(s_{C_{2}}^{\mathrm{ct}}-x_{t}^{2} s_{D_{4}}^{\mathrm{ct}}\right) \\
\left.-\frac{x_{t}^{2}}{2} s_{D_{2}}^{\mathrm{ct}}+\left[\frac{\eta}{2}+x_{t}^{2}\left(1-4 U_{\times}\right)\right] s_{D_{3}}^{\mathrm{ct}}\right\}, \\
\Sigma_{H A}^{(2) \mathrm{ct}}=\sum_{\substack{i \neq 1 \\
j \neq i}}^{2} \frac{N_{c} c_{\beta}^{2} h_{t}^{2}}{16 \pi^{2}} \frac{\Im \mathfrak{m}\left[X_{t} \mu^{*}\right]}{s_{\beta} c_{\beta} \Delta_{\tilde{t}_{i} \tilde{t}_{j}}}\left\{\frac{1}{2} s_{A_{3}}^{\mathrm{ct}}-\left(\frac{\eta}{2}-1\right) s_{A_{4}}^{\mathrm{ct}}+\frac{2 m_{t}^{2} \eta}{\Delta_{\tilde{t}_{i} \tilde{t}_{j}} \eta} s_{A_{5}}^{\mathrm{ct}}+\frac{\eta}{2} s_{B_{1}}^{\mathrm{ct}}\right.
\end{gathered}
$$$$
+\frac{1}{2} s_{B_{2}}^{\mathrm{ct}}-\frac{s_{\beta} c_{\beta} \Delta_{\tilde{t}_{i} \tilde{t}_{j}}}{\Im \mathfrak{m}\left[X_{t} \mu^{*}\right]}\left[\left(\frac{\eta^{2}}{2}-x_{t}^{2} y_{t}^{2}\right) s_{B_{3}}^{\mathrm{ct}}+\frac{\eta}{2} s_{B_{4}}^{\mathrm{ct}}\right]
$$$$
+\frac{4 m_{t}^{2}}{\Delta_{\tilde{t}_{i} \tilde{t}_{j}}}\left(\eta+x_{t}^{2}\right) s_{C_{1}}^{\mathrm{ct}}-\left(\eta+x_{t}^{2}-1\right) s_{C_{2}}^{\mathrm{ct}}
$$$$
\left.-\frac{2 m_{t}^{2}}{\Delta_{\tilde{t}_{i} \tilde{t}_{j}}} x_{t}^{2}\left(s_{D_{1}}^{\mathrm{ct}}+\eta s_{D_{3}}^{\mathrm{ct}}\right)+\frac{x_{t}^{2}}{2} s_{D_{2}}^{\mathrm{ct}}+\left(\frac{\eta}{2}-1\right) x_{t}^{2} s_{D_{4}}^{\mathrm{ct}}\right\},
$$ 


$$
\begin{aligned}
& \Sigma_{H^{ \pm} H^{ \pm}}^{(2) \mathrm{ct}}=\frac{N_{c} c_{\beta}^{2} h_{t}^{2}}{16 \pi^{2}}\left\{s_{E}^{\mathrm{ct}}-s_{F_{1}}^{\mathrm{ct}}-s_{F_{2}}^{\mathrm{ct}}\right\} \\
& +\sum_{\substack{i=1 \\
j \neq i}}^{2} \frac{N_{c} c_{\beta}^{2} h_{t}^{2}}{16 \pi^{2}}\left\{s_{G}^{\mathrm{ct}}+s_{H}^{\mathrm{ct}}+\frac{m_{t}^{2}\left(\eta-\left|\mathbf{U}_{\tilde{t} 1 i i}\right|^{2}\right)-Y_{t}^{2}\left|\mathbf{U}_{\tilde{t} 1 j}\right|^{2}}{\Delta_{\tilde{t}_{i} \tilde{b}_{1}}} s_{I}^{\mathrm{ct}}+s_{J}^{\mathrm{ct}}+s_{K}^{\mathrm{ct}}+s_{L_{1}}^{\mathrm{ct}}+s_{L_{2}}^{\mathrm{ct}}\right. \\
& \left.+s_{M_{1}}^{\mathrm{ct}}+s_{M_{2}}^{\mathrm{ct}}\right\} \text {. } \\
& s_{A_{1}}^{\mathrm{ct}}=2\left[\frac{\delta^{(1)} h_{t}}{h_{t}}+\frac{\delta^{(1)} Z_{\mathcal{H}_{2}}}{2}\right]\left[-2 A_{0}\left(m_{t}^{2}\right)+\sum_{i=1}^{2} A_{0}\left(m_{\tilde{t}_{i}}^{2}\right)\right] \\
& -4 m_{t}^{2} \frac{\delta^{(1)} m_{t}}{m_{t}} B_{0}\left(0, m_{t}^{2}, m_{t}^{2}\right)+\sum_{i=1}^{2} \delta^{(1)} m_{\tilde{t}_{i} \tilde{t}_{i}}^{2} B_{0}\left(0, m_{\tilde{t}_{i}}^{2}, m_{\tilde{t}_{i}}^{2}\right), \\
& s_{A_{2}}^{\mathrm{ct}}=-8 m_{t}^{2}\left[\frac{\delta^{(1)} m_{t}}{m_{t}}+\frac{\delta^{(1)} h_{t}}{h_{t}}+\frac{\delta^{(1)} Z_{\mathcal{H}_{2}}}{2}\right] B_{0}\left(0, m_{t}^{2}, m_{t}^{2}\right)-16 m_{t}^{4} \frac{\delta^{(1)} m_{t}}{m_{t}} C_{0}\left(0,0,0, m_{t}^{2}, m_{t}^{2}, m_{t}^{2}\right), \\
& s_{A_{3}}^{\mathrm{ct}}=4 m_{t}^{2} \frac{\delta^{(1)} m_{t}}{m_{t}} B_{0}\left(0, m_{\tilde{t}_{i}}^{2}, m_{\tilde{t}_{i}}^{2}\right), \\
& s_{A_{4}}^{\mathrm{ct}}=4 m_{t}^{2}\left\{\left[\frac{\delta^{(1)} h_{t}}{h_{t}}+\frac{\delta^{(1)} Z_{\mathcal{H}_{2}}}{2}\right] B_{0}\left(0, m_{\tilde{t}_{i}}^{2}, m_{\tilde{t}_{i}}^{2}\right)+\delta^{(1)} m_{\tilde{t}_{i} \tilde{t}_{i}}^{2} C_{0}\left(0,0,0, m_{\tilde{t}_{i}}^{2}, m_{\tilde{t}_{i}}^{2}, m_{\tilde{t}_{i}}^{2}\right)\right\}, \\
& s_{A_{5}}^{\text {ct }}=2\left[\frac{\delta^{(1)} h_{t}}{h_{t}}+\frac{\delta^{(1)} Z_{\mathcal{H}_{2}}}{2}\right] A_{0}\left(m_{\tilde{t}_{i}}^{2}\right)-\delta^{(1)} m_{\tilde{t}_{i} \tilde{t}_{i}}^{2}\left[B_{0}\left(0, m_{\tilde{t}_{i}}^{2}, m_{\hat{t}_{j}}^{2}\right)-B_{0}\left(0, m_{\tilde{t}_{i}}^{2}, m_{\tilde{t}_{i}}^{2}\right)\right] \text {, } \\
& s_{B_{1}}^{\mathrm{ct}}=\frac{4\left(\left|\mathbf{U}_{\tilde{t} 11}\right|^{2}-\left|\mathbf{U}_{\tilde{t} 12}\right|^{2}\right)\left|\mathbf{U}_{\tilde{t} 12}\right|^{2}}{X_{t}^{2}} \Re e\left[\frac{\delta^{(1)} m_{\tilde{t}_{1} \tilde{t}_{2}}^{2} \mathbf{U}_{\tilde{t} 22} \mathbf{U}_{\tilde{t} 11}^{*}}{m_{t} X_{t}}\right]\left[A_{0}\left(m_{\tilde{t}_{i}}^{2}\right)-\frac{\Delta_{\tilde{t}_{i} \tilde{t}_{j}}}{2} B_{0}\left(0, m_{\tilde{t}_{i}}^{2}, m_{\tilde{t}_{i}}^{2}\right)\right], \\
& s_{B_{2}}^{\mathrm{ct}}=\frac{4\left(\left|\mathbf{U}_{\tilde{t} 11}\right|^{2}-\left|\mathbf{U}_{\tilde{t} 12}\right|^{2}\right)\left|\mathbf{U}_{\tilde{t} 12}\right|^{2}}{X_{t}^{2}} \Re\left[\frac{\delta^{(1)} m_{\tilde{t}_{1} \tilde{t}_{2}}^{2} \mathbf{U}_{\tilde{t} 22} \mathbf{U}_{\tilde{t} 11}^{*}}{m_{t} X_{t}}\right] \Delta_{\tilde{t}_{i} \tilde{t}_{j}} B_{0}\left(0, m_{\tilde{t}_{i}}^{2}, m_{\tilde{t}_{i}}^{2}\right), \\
& s_{B_{3}}^{\mathrm{ct}}=\frac{4\left|\mathbf{U}_{\tilde{t} 12}\right|^{2}}{X_{t}^{2}} \Im \mathfrak{m}\left[\frac{\delta^{(1)} m_{\tilde{t}_{1} \tilde{t}_{2}}^{2} \mathbf{U}_{\tilde{t} 22} \mathbf{U}_{\tilde{t} 11}^{*}}{m_{t} X_{t}}\right]\left[A_{0}\left(m_{\tilde{t}_{i}}^{2}\right)-\frac{\Delta_{\tilde{t}_{i} \tilde{t}_{j}}}{2} B_{0}\left(0, m_{\tilde{t}_{i}}^{2}, m_{\tilde{t}_{i}}^{2}\right)\right], \\
& s_{B_{4}}^{\mathrm{ct}}=\frac{2\left|\mathbf{U}_{\tilde{t} 12}\right|^{2}}{X_{t}^{2}} \Im \mathfrak{m}\left[\frac{\delta^{(1)} m_{\tilde{t}_{1} \tilde{t}_{2}}^{2} \mathbf{U}_{\tilde{t} 22} \mathbf{U}_{\tilde{t} 11}^{*}}{m_{t} X_{t}}\right] \Delta_{\tilde{t}_{i} \tilde{t}_{j}} B_{0}\left(0, m_{\tilde{t}_{i}}^{2}, m_{\tilde{t}_{i}}^{2}\right), \\
& s_{C_{1}}^{\mathrm{ct}}=\left[\frac{\delta^{(1)} \mu}{\mu}-\frac{\delta^{(1)} t_{\beta}}{t_{\beta}}\right] A_{0}\left(m_{\tilde{t}_{i}}^{2}\right), \\
& s_{C_{2}}^{\mathrm{ct}}=2 m_{t}^{2}\left[\frac{\delta^{(1)} \mu}{\mu}-\frac{\delta^{(1)} t_{\beta}}{t_{\beta}}\right] B_{0}\left(0, m_{\tilde{t}_{i}}^{2}, m_{\tilde{t}_{i}}^{2}\right), \\
& s_{D_{1}}^{\mathrm{ct}}=\frac{\delta^{(1)} x_{t}^{2}}{x_{t}^{2}} A_{0}\left(m_{t_{i}}^{2}\right) \text {, } \\
& s_{D_{2}}^{\mathrm{ct}}=2 m_{t}^{2} \frac{\delta^{(1)} x_{t}^{2}}{x_{t}^{2}} B_{0}\left(0, m_{\tilde{t}_{i}}^{2}, m_{\tilde{t}_{i}}^{2}\right), \\
& s_{D_{3}}^{\mathrm{ct}}=\frac{s_{\beta} c_{\beta} \Delta_{\tilde{t}_{i} \tilde{j}_{j}} \delta^{(1)} \phi_{X}}{\Im \mathfrak{m}\left[X_{t} \mu^{*}\right]} A_{0}\left(m_{\tilde{t}_{i}}^{2}\right), \\
& s_{D_{4}}^{\mathrm{ct}}=2 m_{t}^{2} \frac{s_{\beta} c_{\beta} \Delta_{\tilde{t}_{i} \tilde{j}_{\tilde{j}}} \delta^{(1)} \phi_{X}}{\Im \mathfrak{m}\left[X_{t} \mu^{*}\right]} B_{0}\left(0, m_{\tilde{t}_{i}}^{2}, m_{\tilde{t}_{i}}^{2}\right) \\
& s_{E}^{\mathrm{ct}}=2\left[\frac{\delta^{(1)} h_{t}}{h_{t}}+\frac{\delta^{(1)} Z_{\mathcal{H}_{2}}}{2}\right]\left[A_{0}\left(m_{\tilde{b}_{1}}^{2}\right)-2 A_{0}\left(m_{t}^{2}\right)+\sum_{\substack{i=1 \\
j \neq i}}^{2}\left|\mathbf{U}_{\tilde{t} 1 j}\right|^{2} A_{0}\left(m_{\tilde{t}_{i}}^{2}\right)\right] \\
& -4 m_{t}^{2} \frac{\delta^{(1)} m_{t}}{m_{t}} B_{0}\left(0, m_{t}^{2}, m_{t}^{2}\right)+\delta^{(1)} m_{\tilde{b}_{1} \tilde{b}_{1}}^{2}\left(1-\frac{\eta m_{t}^{2} \Delta_{\tilde{t}_{1} \tilde{t}_{2}}}{\Delta_{\tilde{t}_{1} \tilde{b}_{1}} \Delta_{\tilde{t}_{2} \tilde{b}_{1}}}\right) B_{0}\left(0, m_{\tilde{b}_{1}}^{2}, m_{\tilde{b}_{1}}^{2}\right),
\end{aligned}
$$




$$
\begin{aligned}
& s_{F_{1}}^{\mathrm{ct}}=\frac{\Delta_{\tilde{t}_{1} \tilde{t}_{2}}^{2}\left[2 x_{t}^{2}\left(m_{t}^{2}-Y_{t}^{2}\right)+\Delta_{\tilde{t}_{1} \tilde{t}_{2}} \eta\left(\left|\mathbf{U}_{\tilde{t} 11}\right|^{2}-\left|\mathbf{U}_{\tilde{t} 12}\right|^{2}\right)\right]}{\Delta_{\tilde{t}_{1} \tilde{b}_{1}} \Delta_{\tilde{t}_{2} \tilde{b}_{1}}} \frac{\left|\mathbf{U}_{\tilde{t} 12}\right|^{2}}{X_{t}^{2}} \Re\left[\frac{\delta^{(1)} m_{\tilde{t}_{1} \tilde{t}_{2}}^{2} \mathbf{U}_{\tilde{t} 22} \mathbf{U}_{\tilde{t} 11}^{*}}{m_{t} X_{t}}\right] A_{0}\left(m_{\tilde{b}_{1}}^{2}\right), \\
& s_{F_{2}}^{\mathrm{ct}}=\frac{\Delta_{\tilde{t}_{1} \tilde{t}_{2}}^{2}}{\Delta_{\tilde{t}_{1} \tilde{b}_{1}} \Delta_{\tilde{t}_{2} \tilde{b}_{1}}} \frac{2 \Im \mathfrak{m}\left[X_{t} \mu^{*}\right]}{c_{\beta} s_{\beta}} \frac{\left|\mathbf{U}_{\tilde{t} 12}\right|^{2}}{X_{t}^{2}} \Im \mathfrak{m}\left[\frac{\delta^{(1)} m_{\tilde{t}_{1} \tilde{t}_{2}}^{2} \mathbf{U}_{\tilde{t} 22} \mathbf{U}_{\tilde{t} 11}^{*}}{m_{t} X_{t}}\right] A_{0}\left(m_{\tilde{b}_{1}}^{2}\right) \\
& s_{G}^{\text {ct }}=-\frac{\left|\mathbf{U}_{\tilde{t} 1 i}\right|^{2}\left(m_{t}^{2} \Delta_{\tilde{t}_{j} \tilde{b}_{1}}+Y_{t}^{2} \Delta_{\tilde{t}_{i} \tilde{b}_{1}}\right)}{\Delta_{\tilde{t}_{i} \tilde{b}_{1}} \Delta_{\tilde{t}_{j} \tilde{b}_{1}}} \delta^{(1)} m_{\tilde{b}_{1} \tilde{b}_{1}}^{2} B_{0}\left(0, m_{\tilde{b}_{1}}^{2}, m_{\tilde{b}_{1}}^{2}\right), \\
& s_{H}^{\mathrm{ct}}=\left[\left|\mathbf{U}_{\tilde{t} 1 j}\right|^{2}\left(\frac{Y_{t}^{2}}{\Delta_{\tilde{t}_{i} \tilde{b}_{1}}}+1\right)+\frac{m_{t}^{2}\left(\left|\mathbf{U}_{\tilde{t} 1 i}\right|^{2}-\eta\right)}{\Delta_{\tilde{t}_{i} \tilde{b}_{1}}}\right] \delta^{(1)} m_{\tilde{t}_{i} \tilde{t}_{i}}^{2} B_{0}\left(0, m_{\tilde{t}_{i}}^{2}, m_{\tilde{t}_{i}}^{2}\right), \\
& s_{I}^{\mathrm{ct}}=2\left[\frac{\delta^{(1)} h_{t}}{h_{t}}+\frac{\delta^{(1)} Z_{\mathcal{H}_{2}}}{2}+\frac{\delta^{(1)} m_{\tilde{b}_{1} \tilde{b}_{1}}^{2}-\delta^{(1)} m_{\tilde{t}_{i} \tilde{t}_{i}}^{2}}{2 \Delta_{\tilde{t}_{i} \tilde{b}_{1}}}\right]\left[A_{0}\left(m_{\tilde{b}_{1}}^{2}\right)-A_{0}\left(m_{\tilde{t}_{i}}^{2}\right)\right] \text {, } \\
& s_{J}^{\mathrm{ct}}=\frac{\eta-2\left|\mathbf{U}_{\tilde{t} 1 i}\right|^{2}}{\Delta_{\tilde{t}_{i} \tilde{b}_{1}}} m_{t}^{2} \frac{\delta^{(1)} m_{t}}{m_{t}}\left[A_{0}\left(m_{\tilde{b}_{1}}^{2}\right)-A_{0}\left(m_{\tilde{t}_{i}}^{2}\right)\right], \\
& s_{K}^{\mathrm{ct}}=\frac{m_{t}^{2}\left(\eta+2 x_{t}^{2}\right)-\Delta_{\tilde{t}_{i} \tilde{t}_{j}}\left|\mathbf{U}_{\tilde{t} 1 j}\right|^{2}\left(\eta+2 y_{t}^{2}\right)}{\Delta_{\tilde{t}_{i} \tilde{b}_{1}}}\left[\frac{\delta^{(1)} \mu}{\mu}-\frac{\delta^{(1)} t_{\beta}}{t_{\beta}}\right]\left[A_{0}\left(m_{\tilde{b}_{1}}^{2}\right)-A_{0}\left(m_{\tilde{t}_{i}}^{2}\right)\right], \\
& s_{L_{1}}^{\mathrm{ct}}=-\frac{2 m_{t}^{2} x_{t}^{2}-\Delta_{\tilde{t}_{i} \tilde{j}_{j}} \eta\left|\mathbf{U}_{\tilde{t} 1 j}\right|^{2}}{2 \Delta_{\tilde{t}_{i} \tilde{b}_{1}}} \frac{\delta^{(1)} x_{t}^{2}}{x_{t}^{2}}\left[A_{0}\left(m_{\tilde{b}_{1}}^{2}\right)-A_{0}\left(m_{\tilde{t}_{i}}^{2}\right)\right], \\
& s_{L_{2}}^{\mathrm{ct}}=\frac{2 \Im \mathfrak{m}\left[X_{t} \mu^{*}\right]}{c_{\beta} s_{\beta}} \frac{\left|\mathbf{U}_{\tilde{t} 1 j}\right|^{2}}{\Delta_{\tilde{t}_{i} \tilde{b}_{1}}} \delta^{(1)} \phi_{X}\left[A_{0}\left(m_{\tilde{b}_{1}}^{2}\right)-A_{0}\left(m_{\tilde{t}_{i}}^{2}\right)\right], \\
& s_{M_{1}}^{\mathrm{ct}}=\left\{2\left|\mathbf{U}_{\tilde{t} 1 j}\right|^{2}-\frac{\Delta_{\tilde{t}_{i} \tilde{t}_{j}}\left[2 x_{t}^{2}\left(m_{t}^{2}-Y_{t}^{2}\right)+\Delta_{\tilde{t}_{i} \tilde{t}_{j}} \eta U_{-}\right]}{\Delta_{\tilde{t}_{i} \tilde{b}_{1}}} \frac{\left|\mathbf{U}_{\tilde{t} 1 j}\right|^{2}}{X_{t}^{2}}\right\} \Re\left[\frac{\delta^{(1)} m_{\tilde{t}_{i} \tilde{t}_{j}}^{2} \mathbf{U}_{\tilde{t} 2 j} \mathbf{U}_{\tilde{t} 1 i}^{*}}{m_{t} X_{t}}\right] A_{0}\left(m_{\tilde{t}_{i}}^{2}\right), \\
& s_{M_{2}}^{\mathrm{ct}}=-\frac{\Delta_{\tilde{t}_{i} \tilde{t}_{j}}}{\Delta_{\tilde{t}_{i} \tilde{b}_{1}}} \frac{2 \Im \mathfrak{m}\left[X_{t} \mu^{*}\right]}{c_{\beta} s_{\beta}} \frac{\left|\mathbf{U}_{\tilde{t} 1 j}\right|^{2}}{X_{t}^{2}} \Im \mathfrak{m}\left[\frac{\delta^{(1)} m_{\tilde{t}_{i} \tilde{t}_{j}}^{2} \mathbf{U}_{\tilde{t} 2 j} \mathbf{U}_{\tilde{t} 11}^{*}}{m_{t} X_{t}}\right] A_{0}\left(m_{\tilde{t}_{i}}^{2}\right) .
\end{aligned}
$$

\section{D.5 One-loop tadpoles with counterterm insertions}

The one-loop tadpoles with counterterm insertion are part of the two-loop tadpoles of the Higgs bosons. They are given by

$$
\begin{aligned}
T_{h}^{(2) \mathrm{ct}} & =\frac{N_{c} s_{\beta} h_{t} m_{t}}{8 \sqrt{2} \pi^{4}}\left\{t_{A_{1}}^{\mathrm{ct}}+\sum_{\substack{i=1 \\
j \neq i}}^{2}\left[\left(1+x_{t}^{2}\right) t_{A_{2}}^{\mathrm{ct}}+x_{t}^{2} t_{B_{1}}^{\mathrm{ct}}+\frac{x_{t}^{2}}{2} t_{D_{1}}^{\mathrm{ct}}\right]\right\} \\
T_{H}^{(2) \mathrm{ct}} & =\frac{N_{c} c_{\beta} h_{t} m_{t}}{8 \sqrt{2} \pi^{4}}\left\{-t_{A_{1}}^{\mathrm{ct}}+\sum_{\substack{i=1 \\
j \neq i}}^{2}\left[\left(\frac{\eta}{2}-1\right) t_{A_{2}}^{\mathrm{ct}}+\frac{\eta}{2} t_{B_{1}}^{\mathrm{ct}}+\frac{\Im \mathfrak{m}\left[X_{t} \mu^{*}\right]}{s_{\beta} c_{\beta} \Delta_{\tilde{t}_{i} \tilde{t}_{j}}} t_{B_{2}}^{\mathrm{ct}}+\left(\frac{\eta}{2}+x_{t}^{2}\right) t_{C}^{\mathrm{ct}}-\frac{x_{t}^{2}}{2} t_{D_{1}}^{\mathrm{ct}}\right]\right\} \\
T_{A}^{(2) \mathrm{ct}} & =-\sum_{\substack{i=1 \\
j \neq i}}^{2} \frac{N_{c} c_{\beta} h_{t} m_{t}}{8 \sqrt{2} \pi^{4}} \frac{\Im \mathfrak{m}\left[X_{t} \mu^{*}\right]}{s_{\beta} c_{\beta} \Delta_{\tilde{t}_{i} \tilde{t}_{j}}}\left\{t_{A_{2}}^{\mathrm{ct}}+t_{B_{1}}^{\mathrm{ct}}-\frac{s_{\beta} c_{\beta} \Delta_{\tilde{t}_{i} \tilde{t}_{j}}}{\Im \mathfrak{m}\left[X_{t} \mu^{*}\right]} \frac{\eta_{1}}{2} t_{B_{2}}^{\mathrm{ct}}+t_{C}^{\mathrm{ct}}-x_{t}^{2} t_{D_{2}}^{\mathrm{ct}}\right\} \\
t_{A_{1}}^{\mathrm{ct}} & =-2\left[\frac{\delta^{(1)} h_{t}}{h_{t}}+\frac{\delta^{(1)} Z_{\mathcal{H}_{2}}}{2}\right] A_{0}\left(m_{t}^{2}\right)-\frac{\delta^{(1)} m_{t}}{m_{t}}\left(2 A_{0}\left(m_{t}^{2}\right)+4 m_{t}^{2} B_{0}\left(0, m_{t}^{2}, m_{t}^{2}\right)-\sum_{i=1}^{2} A_{0}\left(m_{\tilde{t}_{i}}^{2}\right)\right) \\
t_{A_{2}}^{\mathrm{ct}} & =\left[\frac{\delta^{(1)} h_{t}}{h_{t}}+\frac{\delta^{(1)} Z_{\mathcal{H}_{2}}}{2}\right] A_{0}\left(m_{\tilde{t}_{i}}^{2}\right)+\delta^{(1)} m_{\tilde{t}_{i} \tilde{t}_{i}}^{2} B_{0}\left(0, m_{\tilde{t}_{i}}^{2}, m_{\tilde{t}_{i}}^{2}\right)
\end{aligned}
$$




$$
\begin{aligned}
t_{B_{1}}^{\mathrm{ct}} & =\frac{\left(\left|\mathbf{U}_{\tilde{t} 111}\right|^{2}-\left|\mathbf{U}_{\tilde{t} 12}\right|^{2}\right)\left|\mathbf{U}_{\tilde{t} 12}\right|^{2} \Delta_{\tilde{t}_{i} \tilde{t}_{j}}}{m_{t}^{2} x_{t}^{2}}\left[\frac{\delta^{(1)} m_{\tilde{t}_{1} \tilde{t}_{2}}^{2} \mathbf{U}_{\tilde{t} 22} \mathbf{U}_{\tilde{t} 11}^{*}}{m_{t} X_{t}}\right] A_{0}\left(m_{\tilde{t}_{i}}^{2}\right), \\
t_{B_{2}}^{\mathrm{ct}} & =\frac{\left|\mathbf{U}_{\tilde{t} 12}\right|^{2} \Delta_{\tilde{t}_{i} \tilde{t}_{j}}}{m_{t}^{2} x_{t}^{2}} \Im \mathrm{m}\left[\frac{\delta^{(1)} m_{\tilde{t}_{1} \tilde{t}_{2}}^{2} \mathbf{U}_{\tilde{t} 22} \mathbf{U}_{\tilde{t} 11}^{*}}{m_{t} X_{t}}\right] A_{0}\left(m_{\tilde{t}_{i}}^{2}\right), \\
t_{C}^{\mathrm{ct}} & =s_{C_{1}}^{\mathrm{ct}}, \\
t_{D_{1}}^{\mathrm{ct}} & =s_{D_{1}}^{\mathrm{ct}}, \\
t_{D_{2}}^{\mathrm{ct}} & =s_{D_{3}}^{\mathrm{ct}} .
\end{aligned}
$$

\section{D.6 Renormalization constants for subrenormalization}

The required renormalization constants are explicitly expressed in the following:

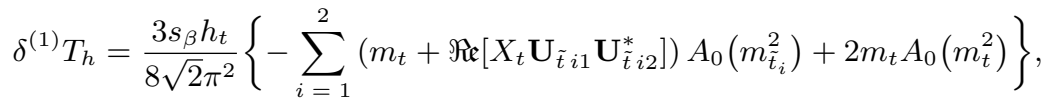

$$
\begin{aligned}
& \delta^{(1)} T_{H}=\frac{3 c_{\beta} h_{t}}{8 \sqrt{2} \pi^{2}}\left\{\sum_{i=1}^{2}\left(m_{t}+\Re \mathfrak{r}\left[Y_{t} \mathbf{U}_{\tilde{t} i 1} \mathbf{U}_{\tilde{t} i 2}^{*}\right]\right) A_{0}\left(m_{\tilde{t}_{i}}^{2}\right)-2 m_{t} A_{0}\left(m_{t}^{2}\right)\right\}, \\
& \delta^{(1)} T_{A}=-\frac{3 c_{\beta} h_{t}}{8 \sqrt{2} \pi^{2}} \sum_{i=1}^{2} \Im \mathfrak{m}\left[Y_{t} \mathbf{U}_{\tilde{t} i 1} \mathbf{U}_{\tilde{t} i 2}^{*}\right] A_{0}\left(m_{\tilde{t}_{i}}^{2}\right), \\
& \delta^{(1)} m_{H^{ \pm}}^{2}=\frac{3 c_{\beta}^{2} h_{t}^{2}}{16 \pi^{2}}\left\{\sum_{i=1}^{2}\left[\left|\mathbf{U}_{\tilde{t} i 2}\right|^{2} A_{0}\left(m_{\tilde{t}_{i}}^{2}\right)+\left|m_{t} \mathbf{U}_{\tilde{t} i 1}^{*}+Y_{t} \mathbf{U}_{\tilde{t} i 2}^{*}\right|^{2} \Re \mathfrak{r}\left[B_{0}\left(0, m_{\tilde{t}_{i}}^{2}, m_{\tilde{b}_{1}}^{2}\right)\right]\right]\right. \\
& \left.-2 m_{t}^{2} \Re \mathfrak{e}\left[B_{0}\left(0,0, m_{t}^{2}\right)\right]+A_{0}\left(m_{\tilde{b}_{1}}^{2}\right)\right\}, \\
& \delta^{(1)} Z_{\mathcal{H}_{1}}=0, \\
& \delta^{(1)} Z_{\mathcal{H}_{2}}=-\frac{3 h_{t}^{2}}{16 \pi^{2} \epsilon}, \\
& \frac{\delta^{(1)} M_{W}^{2}}{M_{W}^{2}}=\frac{3 s_{\beta}^{2}}{16 \pi^{2}} \frac{h_{t}^{2}}{m_{t}^{2}}\left\{2\left(2 \Re \mathfrak{R}\left[B_{00}\left(0,0, m_{t}^{2}\right)\right]-m_{t}^{2} \Re \mathfrak{e}\left[B_{0}\left(0,0, m_{t}^{2}\right)\right]\right)+A_{0}\left(m_{\tilde{b}_{1}}^{2}\right)\right. \\
& \left.+\sum_{i=1}^{2}\left|\mathbf{U}_{\tilde{t} i 1}\right|^{2}\left(A_{0}\left(m_{\tilde{t}_{i}}^{2}\right)-4 \Re e\left[B_{00}\left(0, m_{\tilde{b}_{1}}^{2}, m_{\tilde{t}_{i}}^{2}\right)\right]\right)\right\}, \\
& \frac{\delta^{(1)} M_{Z}^{2}}{M_{Z}^{2}}=\frac{3 s_{\beta}^{2}}{16 \pi^{2}} \frac{h_{t}^{2}}{m_{t}^{2}}\left\{-m_{t}^{2} \Re \mathfrak{e}\left[B_{0}\left(0, m_{t}^{2}, m_{t}^{2}\right)\right]+\sum_{i=1}^{2}\left|\mathbf{U}_{\tilde{t} i 1}\right|^{2}\left|\mathbf{U}_{\tilde{t} i 2}\right|^{2} A_{0}\left(m_{\tilde{t}_{i}}^{2}\right)\right. \\
& \left.-4 \Re \mathfrak{R}\left[B_{00}\left(0, m_{\tilde{t}_{1}}^{2}, m_{\tilde{t}_{2}}^{2}\right)\right]\left|\mathbf{U}_{\tilde{t} 11}\right|^{2}\left|\mathbf{U}_{\tilde{t} 12}\right|^{2}\right\}, \\
& \frac{\delta^{(1)} m_{t}}{m_{t}}=\frac{h_{t}^{2}}{32 \pi^{2}}\left\{-\Re e\left[B_{1}\left(m_{t}^{2}, \mu^{2}, m_{\tilde{t}_{1}}^{2}\right)+B_{1}\left(m_{t}^{2}, \mu^{2}, m_{\tilde{t}_{2}}^{2}\right)+B_{1}\left(m_{t}^{2}, \mu^{2}, m_{\tilde{b}_{1}}^{2}\right)\right]\right. \\
& \left.+c_{\beta}^{2} \frac{\delta^{(1)} m_{t}^{H}}{m_{t}}+\left.s_{\beta}^{2} \frac{\delta^{(1)} m_{t}^{H}}{m_{t}}\right|_{m_{H^{ \pm}} \rightarrow 0}\right\} \\
& \frac{\delta^{(1)} m_{t}^{H}}{m_{t}}=\Re e\left[B_{0}\left(m_{t}^{2}, m_{H^{ \pm}}^{2}, m_{t}^{2}\right)+B_{1}\left(m_{t}^{2}, m_{H^{ \pm}}^{2}, m_{t}^{2}\right)-B_{1}\left(m_{t}^{2}, 0, m_{H^{ \pm}}^{2}\right)+B_{1}\left(m_{t}^{2}, m_{t}^{2}, m_{H^{ \pm}}^{2}\right)\right], \\
& \delta^{(1)} m_{\tilde{t}_{i} \tilde{t}_{i}}^{2}=\frac{h_{t}^{2}}{16 \pi^{2}}\left\{-2 m_{\tilde{t}_{i}}^{2} \Re e\left[B_{1}\left(m_{\tilde{t}_{i}}^{2}, m_{t}^{2}, \mu^{2}\right)\right]+8 U_{\times} A_{0}\left(m_{\tilde{t}_{i}}^{2}\right)+\left(1-8 U_{\times}\right) A_{0}\left(m_{\tilde{t}_{j}}^{2}\right)\right. \\
& -2\left(\left|\mathbf{U}_{\tilde{t} 1 j}\right|^{2}+1\right) A_{0}\left(\mu^{2}\right)+\left|\mathbf{U}_{\tilde{t} 1 j}\right|^{2} A_{0}\left(m_{\tilde{b}_{1}}^{2}\right) \\
& -2 m_{t}^{2} \Re \mathfrak{e}\left[B_{0}\left(m_{\tilde{t}_{i}}^{2}, m_{t}^{2}, \mu^{2}\right)\right]-2 m_{\tilde{t}_{i}}^{2}\left|\mathbf{U}_{\tilde{t} 1 j}\right|^{2} \Re \mathfrak{e}\left[B_{1}\left(m_{\tilde{t}_{i}}^{2}, 0, \mu^{2}\right)\right] \\
& \left.+c_{\beta}^{2} \delta^{(1)} m_{\tilde{t}_{i} \tilde{t}_{i}}^{2 H}+\left.s_{\beta}^{2} \delta^{(1)} m_{\tilde{t}_{i} \tilde{t}_{i}}^{2 H}\right|_{m_{H^{ \pm}} \rightarrow 0, Y_{t}^{2} \rightarrow X_{t}^{2}, y_{t}^{2} \rightarrow x_{t}^{2}, \eta \rightarrow-2 x_{t}^{2}}\right\}, \quad j \neq i,
\end{aligned}
$$




$$
\begin{aligned}
& \delta^{(1)} m_{\tilde{t}_{i} \tilde{t}_{i}}^{2 H}=Y_{t}^{2}\left(1-2 U_{\times}\right) \Re\left[\left[B_{0}\left(m_{\tilde{t}_{i}}^{2}, m_{H^{ \pm}}^{2}, m_{\tilde{t}_{j}}^{2}\right)\right]-2 m_{t}^{2}\left(\eta-x_{t}^{2} y_{t}^{2}-1\right) \Re \mathfrak{R e}\left[B_{0}\left(m_{\tilde{t}_{i}}^{2}, m_{H^{ \pm}}^{2}, m_{\tilde{t}_{i}}^{2}\right)\right]\right. \\
& +\left(m_{t}^{2}\left|\mathbf{U}_{\tilde{t} 1 i}\right|^{2}+Y_{t}^{2}\left|\mathbf{U}_{\tilde{t} 1 j}\right|^{2}-\eta m_{t}^{2}\right) \Re\left[B_{0}\left(m_{\tilde{t}_{i}}^{2}, m_{H^{ \pm}}^{2}, m_{\tilde{b}_{1}}^{2}\right)\right]+\left(\left|\mathbf{U}_{\tilde{t} 1 j}\right|^{2}+1\right) A_{0}\left(m_{H^{ \pm}}^{2}\right), \\
& \delta^{(1)} m_{\tilde{t}_{1} \tilde{t}_{2}}^{2}=\frac{h_{t}^{2} \mathbf{U}_{\tilde{t} 11} \mathbf{U}_{\tilde{t} 22}^{*}\left|\mathbf{U}_{\tilde{t} 12}\right|^{2} \Delta_{\tilde{t}_{1} \tilde{t}_{2}}}{16 \pi^{2} m_{t} X_{t}^{*}}\left\{\sum_{i=1}^{2}\left[4 U_{-} A_{0}\left(m_{\tilde{t}_{i}}^{2}\right)-m_{\tilde{t}_{i}}^{2} \Re \mathfrak{k}\left[B_{1}\left(m_{\tilde{t}_{i}}^{2}, 0, \mu^{2}\right)\right]\right]\right. \\
& -2 A_{0}\left(\mu^{2}\right)+A_{0}\left(m_{\tilde{b}_{1}}^{2}\right) \\
& -\frac{i c_{\beta}^{2} \Im \mathfrak{m}\left[X_{t} \mu^{*}\right] \Delta_{\tilde{t}_{1} \tilde{t}_{2}}}{2 c_{\beta} s_{\beta} X_{t}^{2}} \sum_{i, j=1}^{2}\left\{\Re \mathfrak{e}\left[B_{0}\left(m_{\tilde{t}_{i}}^{2}, m_{H^{ \pm}}^{2}, m_{\tilde{t}_{j}}^{2}\right)\right]\right. \\
& \left.+\Re \mathfrak{R}\left[B_{0}\left(m_{\tilde{t}_{i}}^{2}, m_{H^{ \pm}}^{2}, m_{\tilde{b}_{1}}^{2}\right)\right]\right\} \\
& \left.+c_{\beta}^{2} \delta^{(1)} m_{\tilde{t}_{1} \tilde{t}_{2}}^{2 H}+\left.s_{\beta}^{2} \delta^{(1)} m_{\tilde{t}_{1} \tilde{t}_{2}}^{2 H}\right|_{m_{H^{ \pm}} \rightarrow 0, y_{t}^{2} \rightarrow x_{t}^{2}, \eta \rightarrow-2 x_{t}^{2}}\right\} \text {, } \\
& \delta^{(1)} m_{\tilde{t}_{1} \tilde{t}_{2}}^{2 H}=A_{0}\left(m_{H^{ \pm}}^{2}\right)-\frac{\Delta_{\tilde{t}_{1} \tilde{t}_{2}}^{2}}{2 X_{t}^{2}} \sum_{\substack{i=1 \\
j \neq i}}^{2}\left[\frac{\eta U_{-}}{2}-x_{t}^{2} y_{t}^{2}+U_{\times}\right] \Re \mathfrak{R}\left[B_{0}\left(m_{\tilde{t}_{i}}^{2}, m_{H^{ \pm}}^{2}, m_{\tilde{b}_{1}}^{2}\right)\right] \\
& -\frac{\Delta_{\tilde{t}_{1} \tilde{t}_{2}}^{2}}{2 X_{t}^{2}} \sum_{\substack{i=1 \\
j \neq i}}^{2} U_{-}\left[\frac{\eta}{2}-x_{t}^{2} y_{t}^{2}\right] \Re\left[B_{0}\left(m_{\tilde{t}_{i}}^{2}, m_{H^{ \pm}}^{2}, m_{\tilde{t}_{i}}^{2}\right)+B_{0}\left(m_{\tilde{t}_{j}}^{2}, m_{H^{ \pm}}^{2}, m_{\tilde{t}_{i}}^{2}\right)\right], \\
& \delta^{(1)} m_{\tilde{t}_{2} \tilde{t}_{1}}^{2}=\left(\delta^{(1)} m_{\tilde{t}_{1} \tilde{t}_{2}}^{2}\right)^{*} \text {, } \\
& \frac{\delta^{(1)} \mu}{\mu}=-\frac{3 h_{t}^{2}}{32 \pi^{2}}\left\{\Re \mathfrak{e}\left[B_{1}\left(\mu^{2}, m_{t}^{2}, m_{\tilde{b}_{1}}^{2}\right)\right]+\sum_{i=1}^{2}\left|\mathbf{U}_{\tilde{t} i 2}\right|^{2} \Re \mathfrak{e}\left[B_{1}\left(\mu^{2}, 0, m_{\tilde{t}_{i}}^{2}\right)\right]\right\} .
\end{aligned}
$$

Open Access. This article is distributed under the terms of the Creative Commons Attribution License (CC-BY 4.0), which permits any use, distribution and reproduction in any medium, provided the original author(s) and source are credited.

\section{References}

[1] ATLAS collaboration, Observation of a new particle in the search for the standard model Higgs boson with the ATLAS detector at the LHC, Phys. Lett. B 716 (2012) 1 [arXiv: 1207.7214] [INSPIRE].

[2] CMS collaboration, Observation of a new boson at a mass of $125 \mathrm{GeV}$ with the CMS experiment at the LHC, Phys. Lett. B 716 (2012) 30 [arXiv:1207.7235] [INSPIRE].

[3] ATLAS collaboration, M. Kado, Physics of the Brout-Englert-Higgs boson in ATLAS, talk given at the $37^{\text {th }}$ International Conference on High Energy Physics, Valencia Spain July 2014.

[4] CMS collaboration, A. David, Physics of the Brout-Englert-Higgs boson in CMS, talk given at the $37^{\text {th }}$ International Conference on High Energy Physics, Valencia Spain July 2014.

[5] A. Pilaftsis, CP odd tadpole renormalization of Higgs scalar-pseudoscalar mixing, Phys. Rev. D 58 (1998) 096010 [hep-ph/9803297] [INSPIRE].

[6] A. Pilaftsis, Higgs scalar-pseudoscalar mixing in the minimal supersymmetric standard model, Phys. Lett. B 435 (1998) 88 [hep-ph/9805373] [INSPIRE]. 
[7] J.A. Casas, J.R. Espinosa, M. Quirós and A. Riotto, The lightest Higgs boson mass in the minimal supersymmetric standard model, Nucl. Phys. B 436 (1995) 3 [Erratum ibid. B 439 (1995) 466] [hep-ph/9407389] [INSPIRE].

[8] M.S. Carena, J.R. Espinosa, M. Quirós and C.E.M. Wagner, Analytical expressions for radiatively corrected Higgs masses and couplings in the MSSM, Phys. Lett. B 355 (1995) 209 [hep-ph/9504316] [INSPIRE].

[9] S. Heinemeyer, W. Hollik and G. Weiglein, QCD corrections to the masses of the neutral CP even Higgs bosons in the MSSM, Phys. Rev. D 58 (1998) 091701 [hep-ph/9803277] [INSPIRE].

[10] S. Heinemeyer, W. Hollik and G. Weiglein, Precise prediction for the mass of the lightest Higgs boson in the MSSM, Phys. Lett. B 440 (1998) 296 [hep-ph/9807423] [INSPIRE].

[11] S. Heinemeyer, W. Hollik and G. Weiglein, The masses of the neutral CP even Higgs bosons in the MSSM: accurate analysis at the two loop level, Eur. Phys. J. C 9 (1999) 343 [hep-ph/9812472] [INSPIRE].

[12] S. Heinemeyer, W. Hollik and G. Weiglein, The mass of the lightest MSSM Higgs boson: a compact analytical expression at the two loop level, Phys. Lett. B 455 (1999) 179 [hep-ph/9903404] [INSPIRE].

[13] M.S. Carena et al., Reconciling the two loop diagrammatic and effective field theory computations of the mass of the lightest CP even Higgs boson in the MSSM, Nucl. Phys. B $\mathbf{5 8 0}$ (2000) 29 [hep-ph/0001002] [INSPIRE].

[14] S. Heinemeyer, W. Hollik, H. Rzehak and G. Weiglein, High-precision predictions for the MSSM Higgs sector at $O\left(\alpha_{b} \alpha_{s}\right)$, Eur. Phys. J. C 39 (2005) 465 [hep-ph/0411114] [INSPIRE].

[15] S. Borowka, T. Hahn, S. Heinemeyer, G. Heinrich and W. Hollik, Momentum-dependent two-loop QCD corrections to the neutral Higgs-boson masses in the MSSM, Eur. Phys. J. C 74 (2014) 2994 [arXiv: 1404.7074] [inSPIRE].

[16] R.V. Harlander, P. Kant, L. Mihaila and M. Steinhauser, Higgs boson mass in supersymmetry to three loops, Phys. Rev. Lett. 100 (2008) 191602 [arXiv:0803.0672] [INSPIRE].

[17] R.V. Harlander, P. Kant, L. Mihaila and M. Steinhauser, Higgs boson mass in supersymmetry to three loops, Phys. Rev. Lett. 100 (2008) 191602 [arXiv:0803.0672] [INSPIRE].

[18] P. Kant, R.V. Harlander, L. Mihaila and M. Steinhauser, Light MSSM Higgs boson mass to three-loop accuracy, JHEP 08 (2010) 104 [arXiv: 1005.5709] [INSPIRE].

[19] R.-J. Zhang, Two loop effective potential calculation of the lightest CP even Higgs boson mass in the MSSM, Phys. Lett. B 447 (1999) 89 [hep-ph/9808299] [INSPIRE].

[20] J.R. Espinosa and R.-J. Zhang, Complete two loop dominant corrections to the mass of the lightest CP even Higgs boson in the minimal supersymmetric standard model, Nucl. Phys. B 586 (2000) 3 [hep-ph/0003246] [INSPIRE].

[21] J.R. Espinosa and R.-J. Zhang, MSSM lightest CP even Higgs boson mass to $O\left(\alpha_{s} \alpha_{t}\right)$ : the effective potential approach, JHEP 03 (2000) 026 [hep-ph/9912236] [INSPIRE].

[22] J.R. Espinosa and I. Navarro, Radiative corrections to the Higgs boson mass for a hierarchical stop spectrum, Nucl. Phys. B 615 (2001) 82 [hep-ph/0104047] [INSPIRE].

[23] G. Degrassi, P. Slavich and F. Zwirner, On the neutral Higgs boson masses in the MSSM for arbitrary stop mixing, Nucl. Phys. B 611 (2001) 403 [hep-ph/0105096] [INSPIRE]. 
[24] R. Hempfling and A.H. Hoang, Two loop radiative corrections to the upper limit of the lightest Higgs boson mass in the minimal supersymmetric model, Phys. Lett. B 331 (1994) 99 [hep-ph/9401219] [INSPIRE].

[25] A. Brignole, G. Degrassi, P. Slavich and F. Zwirner, On the two loop sbottom corrections to the neutral Higgs boson masses in the MSSM, Nucl. Phys. B 643 (2002) 79 [hep-ph/0206101] [INSPIRE].

[26] A. Dedes, G. Degrassi and P. Slavich, On the two loop Yukawa corrections to the MSSM Higgs boson masses at large $\tan \beta$, Nucl. Phys. B 672 (2003) 144 [hep-ph/0305127] [INSPIRE].

[27] J.R. Espinosa and R.-J. Zhang, Complete two loop dominant corrections to the mass of the lightest CP even Higgs boson in the minimal supersymmetric standard model, Nucl. Phys. B 586 (2000) 3 [hep-ph/0003246] [INSPIRE].

[28] A. Brignole, G. Degrassi, P. Slavich and F. Zwirner, On the $O\left(\alpha_{t}^{2}\right)$ two loop corrections to the neutral Higgs boson masses in the MSSM, Nucl. Phys. B 631 (2002) 195 [hep-ph/0112177] [INSPIRE].

[29] G. Degrassi, S. Heinemeyer, W. Hollik, P. Slavich and G. Weiglein, Towards high precision predictions for the MSSM Higgs sector, Eur. Phys. J. C 28 (2003) 133 [hep-ph/0212020] [INSPIRE].

[30] S. Heinemeyer, W. Hollik and G. Weiglein, Electroweak precision observables in the minimal supersymmetric standard model, Phys. Rept. 425 (2006) 265 [hep-ph/0412214] [INSPIRE].

[31] B.C. Allanach, A. Djouadi, J.L. Kneur, W. Porod and P. Slavich, Precise determination of the neutral Higgs boson masses in the MSSM, JHEP 09 (2004) 044 [hep-ph/0406166] [INSPIRE].

[32] S.P. Martin, Two loop effective potential for a general renormalizable theory and softly broken supersymmetry, Phys. Rev. D 65 (2002) 116003 [hep-ph/0111209] [INSPIRE].

[33] S.P. Martin, Two loop effective potential for the minimal supersymmetric standard model, Phys. Rev. D 66 (2002) 096001 [hep-ph/0206136] [INSPIRE].

[34] S.P. Martin, Complete two loop effective potential approximation to the lightest Higgs scalar boson mass in supersymmetry, Phys. Rev. D 67 (2003) 095012 [hep-ph/0211366] [INSPIRE].

[35] S.P. Martin, Evaluation of two loop selfenergy basis integrals using differential equations, Phys. Rev. D 68 (2003) 075002 [hep-ph/0307101] [INSPIRE].

[36] S.P. Martin, Two loop scalar self energies in a general renormalizable theory at leading order in gauge couplings, Phys. Rev. D 70 (2004) 016005 [hep-ph/0312092] [INSPIRE].

[37] S.P. Martin, Strong and Yukawa two-loop contributions to Higgs scalar boson self-energies and pole masses in supersymmetry, Phys. Rev. D 71 (2005) 016012 [hep-ph/0405022] [INSPIRE].

[38] S.P. Martin, Two-loop scalar self-energies and pole masses in a general renormalizable theory with massless gauge bosons, Phys. Rev. D 71 (2005) 116004 [hep-ph/0502168] [INSPIRE].

[39] S.P. Martin and D.G. Robertson, TSIL: a program for the calculation of two-loop self-energy integrals, Comput. Phys. Commun. 174 (2006) 133 [hep-ph/0501132] [INSPIRE].

[40] D.A. Demir, Effects of the supersymmetric phases on the neutral Higgs sector, Phys. Rev. D 60 (1999) 055006 [hep-ph/9901389] [INSPIRE]. 
[41] S.Y. Choi, M. Drees and J.S. Lee, Loop corrections to the neutral Higgs boson sector of the MSSM with explicit CP-violation, Phys. Lett. B 481 (2000) 57 [hep-ph/0002287] [INSPIRE].

[42] T. Ibrahim and P. Nath, Corrections to the Higgs boson masses and mixings from chargino, $W$ and charged Higgs exchange loops and large CP phases, Phys. Rev. D 63 (2001) 035009 [hep-ph/0008237] [INSPIRE].

[43] T. Ibrahim and P. Nath, Neutralino exchange corrections to the Higgs boson mixings with explicit CP-violation, Phys. Rev. D 66 (2002) 015005 [hep-ph/0204092] [INSPIRE].

[44] A. Pilaftsis and C.E.M. Wagner, Higgs bosons in the minimal supersymmetric standard model with explicit CP-violation, Nucl. Phys. B 553 (1999) 3 [hep-ph/9902371] [INSPIRE].

[45] M.S. Carena, J.R. Ellis, A. Pilaftsis and C.E.M. Wagner, Renormalization group improved effective potential for the MSSM Higgs sector with explicit CP-violation, Nucl. Phys. B 586 (2000) 92 [hep-ph/0003180] [INSPIRE].

[46] S. Heinemeyer, W. Hollik, H. Rzehak and G. Weiglein, The Higgs sector of the complex MSSM at two-loop order: QCD contributions, Phys. Lett. B 652 (2007) 300 [arXiv: 0705.0746] [INSPIRE].

[47] M. Frank et al., The Higgs boson masses and mixings of the complex MSSM in the Feynman-diagrammatic approach, JHEP 02 (2007) 047 [hep-ph/0611326] [INSPIRE].

[48] S. Heinemeyer, W. Hollik and G. Weiglein, FeynHiggs: a program for the calculation of the masses of the neutral CP even Higgs bosons in the MSSM, Comput. Phys. Commun. 124 (2000) 76 [hep-ph/9812320] [INSPIRE].

[49] T. Hahn, S. Heinemeyer, W. Hollik, H. Rzehak and G. Weiglein, FeynHiggs 2.7, Nucl. Phys. Proc. Suppl. 205-206 (2010) 152 [arXiv: 1007.0956] [inSPIRE].

[50] W. Hollik and S. Paßehr, Two-loop top-Yukawa-coupling corrections to the Higgs boson masses in the complex MSSM, Phys. Lett. B 733 (2014) 144 [arXiv:1401.8275] [InSPIRE].

[51] S. Dimopoulos and S.D. Thomas, Dynamical relaxation of the supersymmetric CP-violating phases, Nucl. Phys. B 465 (1996) 23 [hep-ph/9510220] [InSPIRE].

[52] R.D. Peccei and H.R. Quinn, CP conservation in the presence of instantons, Phys. Rev. Lett. 38 (1977) 1440 [INSPIRE].

[53] R.D. Peccei and H.R. Quinn, Constraints imposed by CP conservation in the presence of instantons, Phys. Rev. D 16 (1977) 1791 [InSPIRE].

[54] N. Baro, F. Boudjema and A. Semenov, Automatised full one-loop renormalisation of the MSSM. I. The Higgs sector, the issue of $\tan \beta$ and gauge invariance, Phys. Rev. D 78 (2008) 115003 [arXiv: 0807.4668] [INSPIRE].

[55] K.E. Williams, H. Rzehak and G. Weiglein, Higher order corrections to Higgs boson decays in the MSSM with complex parameters, Eur. Phys. J. C 71 (2011) 1669 [arXiv:1103.1335] [INSPIRE].

[56] W. Hollik et al., Renormalization of the minimal supersymmetric standard model, Nucl. Phys. B 639 (2002) 3 [hep-ph/0204350] [INSPIRE].

[57] T. Hahn, Generating Feynman diagrams and amplitudes with FeynArts 3, Comput. Phys. Commun. 140 (2001) 418 [hep-ph/0012260] [INSPIRE]. 
[58] G. Weiglein, R. Mertig, R. Scharf and M. Bohm, Computer algebraic calculation of two loop selfenergies in the electroweak standard model, in New computing techniques in physics research II, La Londe-les-Maures France (1992), pg. 617 [INSPIRE].

[59] T. Hahn and M. Pérez-Victoria, Automatized one loop calculations in four-dimensions and D-dimensions, Comput. Phys. Commun. 118 (1999) 153 [hep-ph/9807565] [InSPIRE].

[60] A. Freitas and D. Stöckinger, Gauge dependence and renormalization of $\tan \beta$ in the MSSM, Phys. Rev. D 66 (2002) 095014 [hep-ph/0205281] [inSPIRE].

[61] M. Sperling, D. Stöckinger and A. Voigt, Renormalization of vacuum expectation values in spontaneously broken gauge theories, JHEP 07 (2013) 132 [arXiv: 1305.1548] [INSPIRE].

[62] M. Sperling, D. Stöckinger and A. Voigt, Renormalization of vacuum expectation values in spontaneously broken gauge theories: two-loop results, JHEP 01 (2014) 068 [arXiv: 1310.7629] [INSPIRE].

[63] T. Hahn, S. Heinemeyer, W. Hollik, H. Rzehak and G. Weiglein, FeynHiggs: a program for the calculation of MSSM Higgs-boson observables - version 2.6.5, Comput. Phys. Commun. 180 (2009) 1426 [INSPIRE].

[64] T. Hahn, S. Heinemeyer, W. Hollik, H. Rzehak and G. Weiglein, Higgs masses and more in the complex MSSM with FeynHiggs, arXiv:0710.4891 [INSPIRE].

[65] M. Frank et al., Charged Higgs boson mass of the MSSM in the Feynman diagrammatic approach, Phys. Rev. D 88 (2013) 055013 [arXiv: 1306.1156] [INSPIRE].

[66] T. Falk and K.A. Olive, Electric dipole moment constraints on phases in the constrained MSSM, Phys. Lett. B 375 (1996) 196 [hep-ph/9602299] [InSPIRE].

[67] T. Falk and K.A. Olive, More on electric dipole moment constraints on phases in the constrained MSSM, Phys. Lett. B 439 (1998) 71 [hep-ph/9806236] [INSPIRE].

[68] T. Ibrahim and P. Nath, The chromoelectric and purely gluonic operator contributions to the neutron electric dipole moment in $N=1$ supergravity, Phys. Lett. B 418 (1998) 98 [hep-ph/9707409] [INSPIRE].

[69] T. Ibrahim and P. Nath, The neutron and the lepton EDMs in MSSM, large CP-violating phases and the cancellation mechanism, Phys. Rev. D 58 (1998) 111301 [Erratum ibid. D 60 (1999) 099902] [hep-ph/9807501] [INSPIRE].

[70] T. Ibrahim and P. Nath, Large CP phases and the cancellation mechanism in EDMs in SUSY, string and brane models, Phys. Rev. D 61 (2000) 093004 [hep-ph/9910553] [INSPIRE].

[71] T. Ibrahim and P. Nath, The neutron and the electron electric dipole moment in $N=1$ supergravity unification, Phys. Rev. D 57 (1998) 478 [Erratum ibid. D 58 (1998) 019901] [Erratum ibid. D 60 (1999) 079903] [Erratum ibid. D 60 (1999) 119901] [hep-ph/9708456] [INSPIRE].

[72] E. Accomando, R.L. Arnowitt and B. Dutta, Grand unification scale CP-violating phases and the electric dipole moment, Phys. Rev. D 61 (2000) 115003 [hep-ph/9907446] [InSPIRE].

[73] A. Bartl, T. Gajdosik, W. Porod, P. Stöckinger and H. Stremnitzer, Electron and neutron electric dipole moments in the constrained MSSM, Phys. Rev. D 60 (1999) 073003 [hep-ph/9903402] [INSPIRE].

[74] V.D. Barger et al., CP violating phases in SUSY, electric dipole moments and linear colliders, Phys. Rev. D 64 (2001) 056007 [hep-ph/0101106] [INSPIRE]. 
[75] A. Masiero and L. Silvestrini, CP violation in low-energy SUSY, in Perspectives on supersymmetry, G.L. Kane ed., World Scientific, Singapore (1997), pg. 423 [hep-ph/9709242] [INSPIRE].

[76] M. Brhlik, G.J. Good and G.L. Kane, Electric dipole moments do not require the CP-violating phases of supersymmetry to be small, Phys. Rev. D 59 (1999) 115004 [hep-ph/9810457] [INSPIRE].

[77] M. Brhlik, L.L. Everett, G.L. Kane and J.D. Lykken, A resolution to the supersymmetric CP problem with large soft phases via D-branes, Phys. Rev. Lett. 83 (1999) 2124 [hep-ph/9905215] [INSPIRE].

[78] G. Passarino and M.J.G. Veltman, One loop corrections for $e^{+} e^{-}$annihilation into $\mu^{+} \mu^{-}$in the Weinberg model, Nucl. Phys. B 160 (1979) 151 [INSPIRE].

[79] G. Weiglein, R. Scharf and M. Böhm, Reduction of general two loop selfenergies to standard scalar integrals, Nucl. Phys. B 416 (1994) 606 [hep-ph/9310358] [INSPIRE].

[80] F.A. Berends and J.B. Tausk, On the numerical evaluation of scalar two loop selfenergy diagrams, Nucl. Phys. B 421 (1994) 456 [InSPIRE]. 